University of Tennessee Health Science Center UTHSC Digital Commons

$11-2013$

\title{
In Vitro Manual Therapy and Biorobotic Simulation of Glenohumeral Joint Mobilization Techniques
}

Hunter Johnson Smith

University of Tennessee Health Science Center

Follow this and additional works at: https://dc.uthsc.edu/dissertations

Part of the Physical Therapy Commons, and the Therapeutics Commons

\section{Recommended Citation}

Smith, Hunter Johnson , "In Vitro Manual Therapy and Biorobotic Simulation of Glenohumeral Joint Mobilization Techniques" (2013). Theses and Dissertations (ETD). Paper 247. http://dx.doi.org/10.21007/ etd.cghs.2013.0294.

This Thesis is brought to you for free and open access by the College of Graduate Health Sciences at UTHSC Digital Commons. It has been accepted for inclusion in Theses and Dissertations (ETD) by an authorized administrator of UTHSC Digital Commons. For more information, please contact jwelch30@uthsc.edu. 


\title{
In Vitro Manual Therapy and Biorobotic Simulation of Glenohumeral Joint Mobilization Techniques
}

\begin{abstract}
Physical therapists (PT) employ mobilization techniques for restoring range of motion to joints. Few studies have attempted to quantify the biomechanics of manual therapy on the glenohumeral $(\mathrm{GH})$ joint. The objectives of this study were to develop an in vitro protocol to determine the biomechanical effects of joint mobilization on the $\mathrm{GH}$ joint, and to then simulate these mobilizations in the University of Tennessee Health Science Center (UTHSC) Joint Implant Biomechanics Laboratory's Robotic Testing Platform (RTP).

The $\mathrm{GH}$ joint is an incredibly shallow socket joint. This gives the joint an unusually large range of motion (ROM) compared to other ball joints. The increased ROM makes the joint unstable and susceptible to injury. The joint is completely surrounded by many muscles for support. The primary stabilizers are the rotator cuff (RC) muscles: subscapularis, supraspinatus, infraspinatus, and teres minor. These muscles were chosen to be simulated for the experiments.

The objective of this study was to develop a protocol for quantifying and comparing GH joint mobilization techniques performed by physical therapists in a human cadaveric model. Two different $\mathrm{GH}$ joint positions were investigated using grade IV non-oscillatory mobilizations. Force data was captured using a six (DOF) load cell; three dimensional (3D) positional data was captured using a camera system with light emitting diodes (LEDs). Most notable differences between joint position and therapists occurred during posterior glide mobilization. In addition to studying other $\mathrm{GH}$ mobilization techniques the protocol can be used to determine structural tissue properties and/or measure effects of shoulder injuries on $\mathrm{GH}$ biomechanics.

A separate robotic protocol was developed to simulate anterior, posterior, and inferior glides on the $\mathrm{GH}$ joint in neutral position. Tests were conducted through $10^{\circ}$ flexion and $10^{\circ}$ extension in neutral rotation, $30^{\circ}$ internal rotation, and $30^{\circ}$ external rotation. External rotation was found to be the stiffest joint configuration in all glide positions; neutral rotation configuration was found to be the least stiff.
\end{abstract}

Two protocols were successfully developed: one for capturing PT's technique in manual therapy, another for simulating PT's manual therapy via a robotic testing platform. Future work can be aimed at expanding the ROM these present protocols study. Additionally, the manual articulation model can be developed into a training tool after gathering in vivo human data from additional experiments using a gait lab. The stated model could then be used to teach therapists particular techniques necessary for clinical treatment.

\section{Document Type}

Thesis

\section{Degree Name}

Master of Science (MS)

\section{Program}

Biomedical Engineering and Imaging

\section{Research Advisor}

Denis DiAngelo, Ph.D.

\section{Keywords}

Biomechanics, Glenohumeral, Mobilization, Robot, Shoulder, Simulation 


\section{Subject Categories}

Analytical, Diagnostic and Therapeutic Techniques and Equipment | Medicine and Health Sciences |

Physical Therapy | Rehabilitation and Therapy | Therapeutics 
IN VITRO MANUAL THERAPY AND BIOROBOTIC SIMULATION OF GLENOHUMERAL JOINT MOBILIZATION TECHNIQUES

\author{
A Dissertation \\ Presented for \\ The Graduate Studies Council \\ The University of Tennessee \\ Health Science Center \\ In Partial Fulfillment \\ Of the Requirements for the Degree \\ Master of Science \\ In the Joint Graduate Program in Biomedical Engineering and Imaging \\ From The University of Tennessee and \\ The University of Memphis
}

By

Hunter Johnson Smith

December 2013 
Copyright (C) 2013 by Hunter Johnson Smith. All rights reserved. 


\section{ACKNOWLEDGEMENTS}

I would like to thank everyone who has helped make my graduate experience possible and enjoyable. First, I would like to think my advisor, Dr. Denis DiAngelo, for all of his guidance and for putting up with me for as long as he has. I would like to think all of my committee members for all of their insight and input: Dr. Brian Kelly, Dr. Richard Kasser, and Dr. Gladius Lewis. Dan Wido, John Simmons, Chad Bennett, Tom Stewart and Kelly Salb also deserve special thanks for all of their help on the projects we worked on while in the lab together. I want to thank all of the members of the Biorobotics Laboratory.

And finally, I want to thank all my family for pushing me to never give up. Without their support and encouragement, I would not be where I am today. 


\begin{abstract}
Physical therapists (PT) employ mobilization techniques for restoring range of motion to joints. Few studies have attempted to quantify the biomechanics of manual therapy on the glenohumeral $(\mathrm{GH})$ joint. The objectives of this study were to develop an in vitro protocol to determine the biomechanical effects of joint mobilization on the $\mathrm{GH}$ joint, and to then simulate these mobilizations in the University of Tennessee Health Science Center (UTHSC) Biorobotic Laboratory's Robotic Testing Platform (RTP).

The GH joint is a shallow socket joint that gives the joint an unusually large range of motion (ROM) compared to other ball joints. The increased ROM makes the joint unstable and susceptible to injury. The joint is completely surrounded by many muscles for support. The primary stabilizers are the rotator cuff (RC) muscles: subscapularis, supraspinatus, infraspinatus, and teres minor. These muscles were chosen to be simulated for the experiments.

The objective of this study was to develop a protocol for quantifying and comparing $\mathrm{GH}$ joint mobilization techniques performed by physical therapists in a human cadaveric model. Two different GH joint positions were investigated using grade IV non-oscillatory mobilizations. Force data were captured using a six degree of freedom (DOF) load cell; three dimensional (3D) positional data were captured using a camera system with light emitting diodes (LEDs). Most notable differences between joint position and therapists occurred during posterior glide mobilization. In addition to studying other $\mathrm{GH}$ mobilization techniques the protocol can be used to determine structural tissue properties and/or measure effects of shoulder injuries on $\mathrm{GH}$ biomechanics.
\end{abstract}

A separate robotic protocol was developed to simulate anterior, posterior, and inferior glides on the $\mathrm{GH}$ joint in neutral position. Tests were conducted through $10^{\circ}$ flexion and $10^{\circ}$ extension in neutral rotation, $30^{\circ}$ internal rotation, and $30^{\circ}$ external rotation. External rotation was found to be the stiffest joint configuration in all glide positions; neutral rotation configuration was found to be the least stiff.

Two protocols were successfully developed: one for capturing PT's technique in manual therapy, another for simulating PT's manual therapy via a robotic testing platform. Future work can be aimed at expanding the ROM these present protocols study. Additionally, the manual articulation model can be developed into a training tool after gathering in vivo human data from additional experiments using a gait lab. The stated model could then be used to teach therapists particular techniques necessary for clinical treatment. 


\section{TABLE OF CONTENTS}

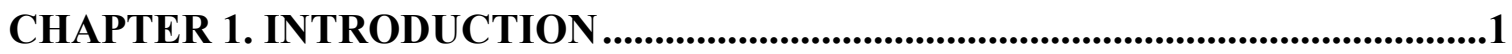

CHAPTER 2. BACKGROUND OF THE GLENOHUMERAL JOINT ......................2

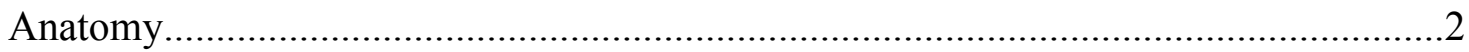

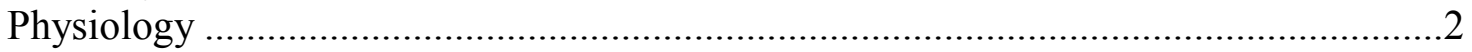

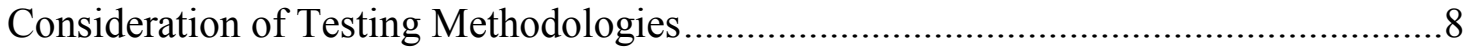

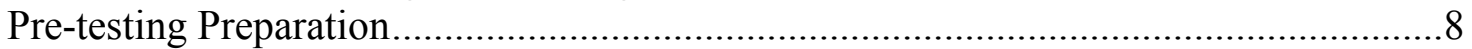

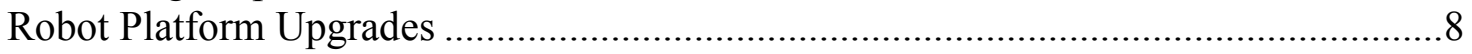

\section{CHAPTER 3. IN VITRO MANUAL THERAPY OF THE GLENOHUMERAL}

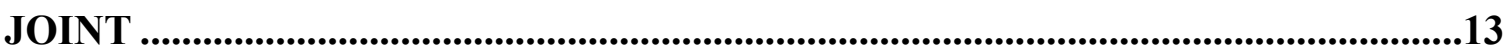

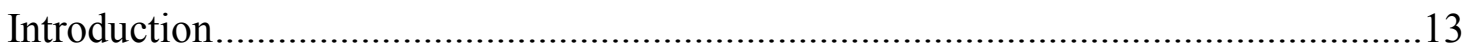

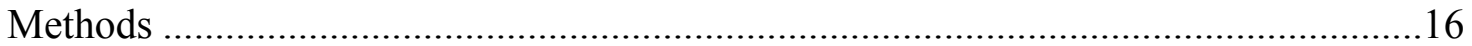

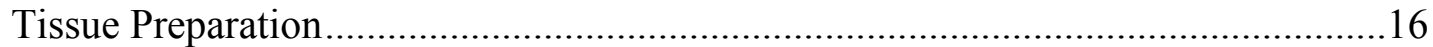

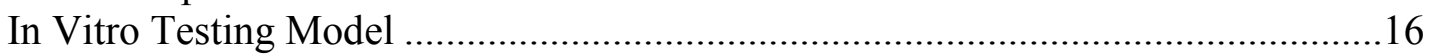

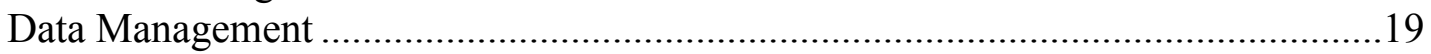

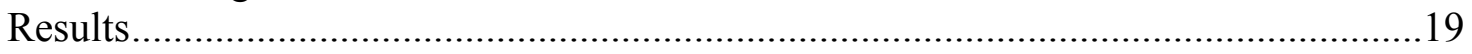

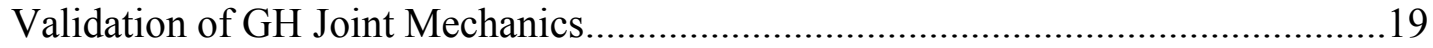

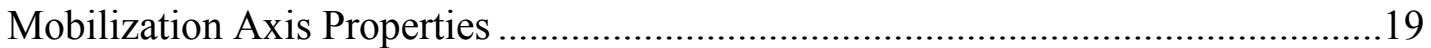

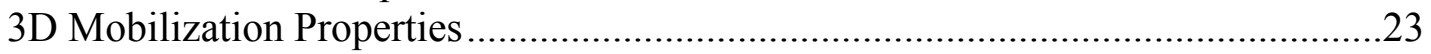

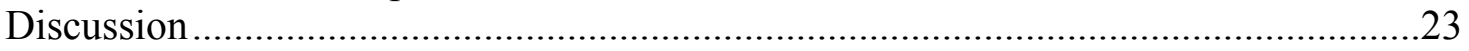

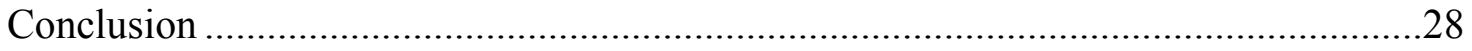

\section{CHAPTER 4. BIOROBOTIC SIMULATION OF GLENOHUMERAL JOINT}

MOBILIZATION TECHNIQUES.....................................................................30

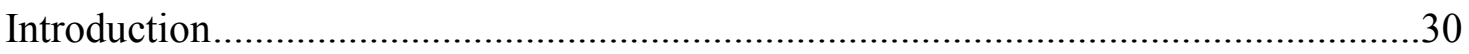

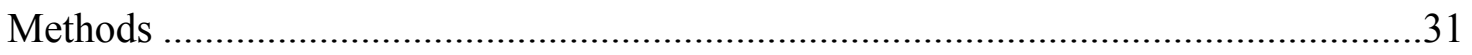

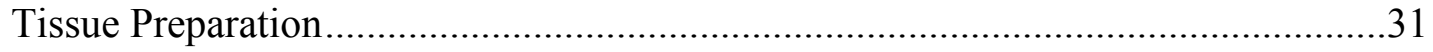

BioRobotic Testing Platform and Specimen Mounting Protocol .............................31

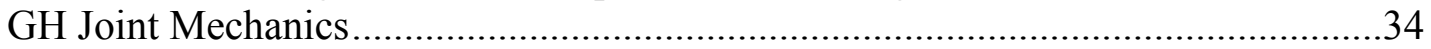

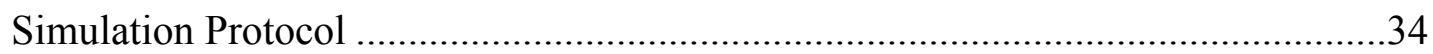

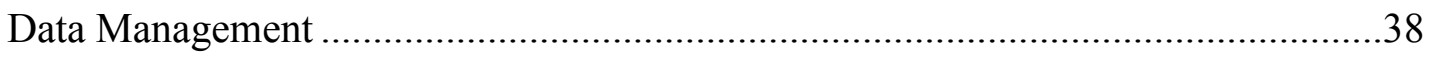

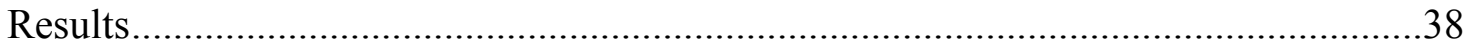

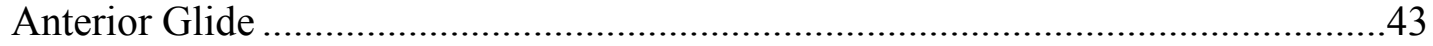

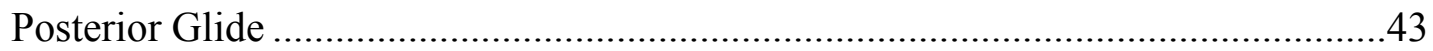

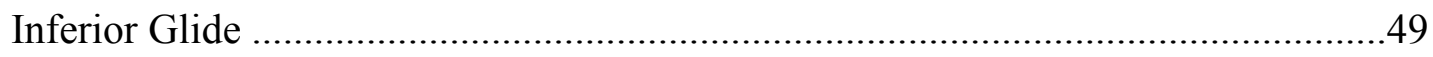

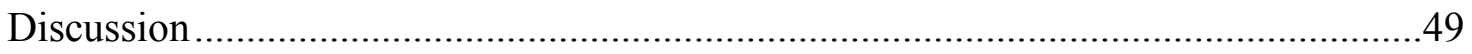

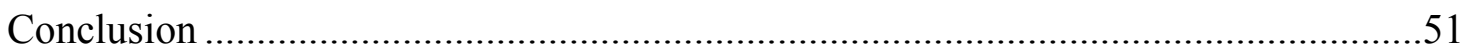

CHAPTER 5. CONCLUSION, LIMITATIONS, AND FUTURE WORK .................52

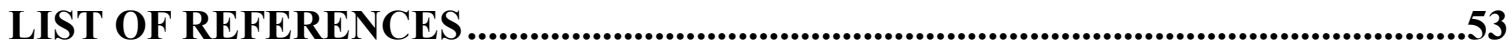


APPENDIX A: SPECIMEN RADIOGRAPHS AND FORCE FRAME

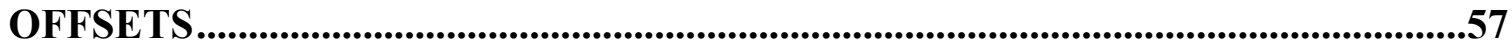

APPENDIX B: PT PROTOCOL DATASET ..........................................................61

APPENDIX C: ROBOTIC PROTOCOL DATASET ...............................................64

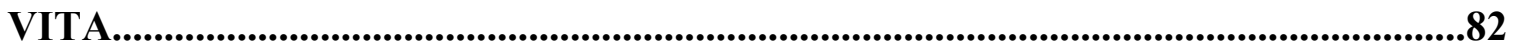




\section{LIST OF TABLES}

Table 2-1. List of muscles involved in shoulder motion..............................................

Table 3-1. Rotational standard deviation during glides (degrees)..................................21

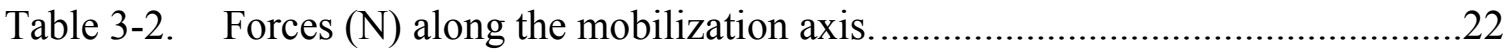

Table 3-3. 3D forces $(\mathrm{N})$, positions $(\mathrm{mm})$, and stiffness values $(\mathrm{N} / \mathrm{mm}) \ldots \ldots \ldots \ldots \ldots \ldots \ldots \ldots . . .25$

Table 3-4. Component forces $(\mathrm{N})$ during posterior resting glide..................................25

Table 4-1. Neutral position 3D stiffness values $(\mathrm{n}=2)$..................................................39

Table 4-2. Mean 3D stiffness values for individual glide paths......................................44

Table B-1. Applied PT forces. ………………………………...................................61

Table B-2. Applied PT displacements. ...................................................................62

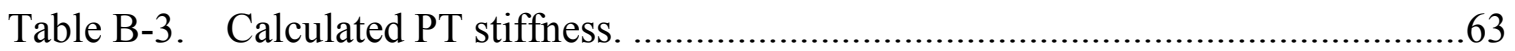

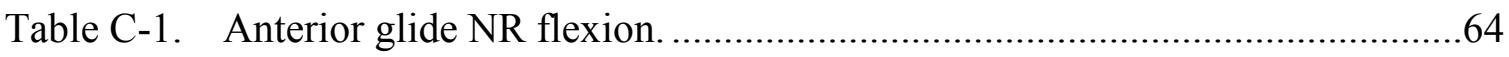

Table C-2. Anterior glide NR extension....................................................................65

Table C-3. Anterior glide 30 degrees IR flexion. .......................................................66

Table C-4. Anterior glide 30 degrees IR extension. ......................................................67

Table C-5. Anterior glide 30 degrees ER flexion. .....................................................68

Table C-6. Anterior glide 30 degrees ER extension. ......................................................69

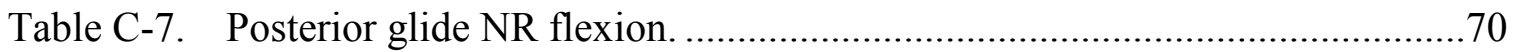

Table C-8. Posterior glide NR extension. .....................................................................71

Table C-9. Posterior glide 30 degrees IR flexion.........................................................72

Table C-10. Posterior glide 30 degrees IR extension......................................................73

Table C-11. Posterior glide 30 degrees ER flexion. …………………………………....74

Table C-12. Posterior glide 30 degrees ER extension. …………………………….......75

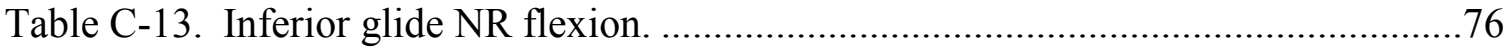

Table C-14. Inferior glide NR extension. .................................................................77 


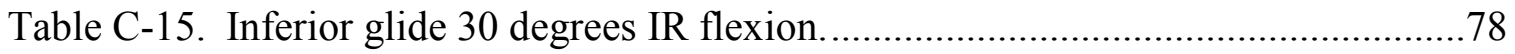

Table C-16. Inferior glide 30 degrees IR extension................................................79

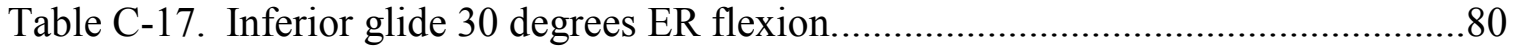

Table C-18. Inferior glide 30 degrees ER extension.....................................................81 


\section{LIST OF FIGURES}

Figure 2-1. Right GH joint................................................................................

Figure 2-2. Sketch of the GH joint and scapula.......................................................6

Figure 2-3. Lateral view of the right shoulder GH joint.........................................

Figure 2-4. Example of a closed loop testing apparatus.......................................

Figure 2-5. Pictures of an open loop testing apparatus...........................................10

Figure 2-6. Computer aided drawing of the robotic testing platform...........................12

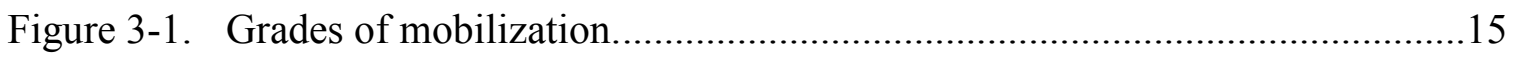

Figure $3-2 . \quad$ Sutured and potted specimen. .......................................................... 17

Figure 3-3. Schematic of coordinate system transformations. ..................................18

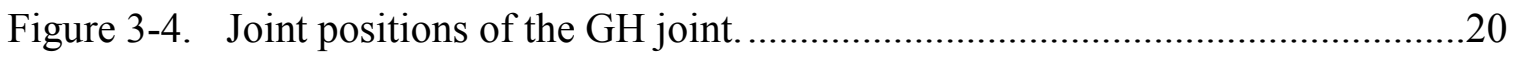

Figure 3-5. Comparison of mobilization axis forces between physical therapists. .........24

Figure 3-6. Analysis of component forces during posterior glide ...............................26

Figure 3-7. Comparison between in vivo and in vitro glides in the anterior and posterior directions

Figure 4-1. Photograph of the prepared GH joint specimen......................................32

Figure 4-2. Arrangement of four controlled axes of the RTP.....................................33

Figure 4-3. Test setup with the specimen mounted and rotator cuff loads engaged. ......35

Figure 4-4. Schematic of the fixture system used to simulate RC muscles.....................36

Figure 4-5. Specimen in premobilized (BP) and postmobilized (DP) position...............37

Figure 4-6. Anterior glide 3D stiffness graph...............................................40

Figure 4-7. Posterior glide 3D stiffness graph.................................................. 41

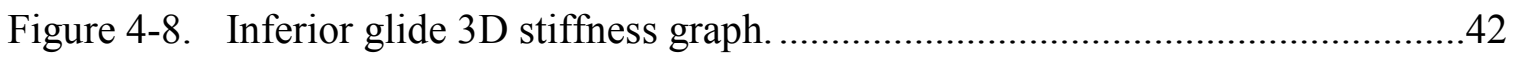

Figure 4-9. Effects of IR/ER on GH joint neutral position before mobilization............45 
Figure 4-10. Effects of IR/ER and F/E on GH joint position before and after an anterior glide mobilization.

Figure 4-11. Effects of IR/ER and F/E on GH joint position before and after a posterior glide mobilization.

Figure 4-12. Effects of IR/ER and F/E on GH joint position before and after an inferior glide mobilization.

Figure 4-13. In vivo comparison between axial GH stiffness values...........................50

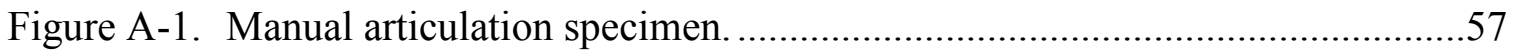

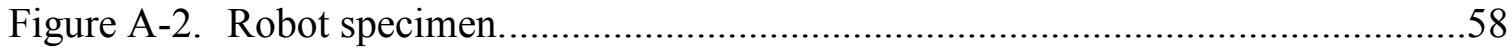

Figure A-3. Manual articulation specimen with force frame offsets............................59

Figure A-4. Robot specimen with force frame offsets. .............................................60 


\section{LIST OF ABBREVIATIONS}

\begin{tabular}{|c|c|}
\hline $\mathrm{A}$ & Anterior \\
\hline BLC & Base Load Cell \\
\hline $\mathrm{BP}$ & Balance Point \\
\hline CS & Coordinate System \\
\hline DOF & Degrees of Freedom \\
\hline DP & Displacement Point \\
\hline $\mathrm{E}$ & Extension \\
\hline ER & External Rotation \\
\hline $\mathrm{F}$ & Flexion \\
\hline $\mathrm{GH}$ & Glenohumeral \\
\hline GLC & Gimbal Load Cell \\
\hline $\mathrm{I}$ & Inferior \\
\hline IR & Internal Rotation \\
\hline $\mathrm{LC}$ & Load Cell \\
\hline LED & Light Emitting Diode \\
\hline MOB & Mobilization \\
\hline NR & No Rotation \\
\hline $\mathrm{P}$ & Posterior \\
\hline PT & Physical Therapist \\
\hline $\mathrm{RC}$ & Rotator Cuff \\
\hline ROM & Range of Motion \\
\hline RTP & Robot Testing Platforn \\
\hline
\end{tabular}


RVDT

UTHSC

WCS

$3 \mathrm{D}$
Rotary Variable Differential Transducer

University of Tennessee Health Science Center

World Coordinate System

Three Dimensional 


\section{CHAPTER 1. INTRODUCTION}

Because of its simplistic structure, the glenohumeral joint is highly mobile and unstable. This high range of motion is crucial in everyday tasks, but the joint's mobility gives rise to the probability of injury. This makes the shoulder joint one of the most injured in the human body (Butcher et al., 1996). Physical therapists treat injuries by mobilizing joints to reduce pain and increase range of motion (Maitland, 1976). This makes the GH joint a prime candidate for clinical joint mobilization therapy. Shoulder dysfunction is the second most common reason for referral to outpatient physical therapy (Vermeulen et al., 2000). Glenohumeral joint hypomobility can contribute directly or indirectly to shoulder pain and immobility (Kelley et al., 2009; Wilk et al., 2009; Brudvig et al., 2011). Physical therapists (PTs) use joint mobilization techniques as the intervention of choice to address capsular hypomobility (Maitland, 1977; Rollins et al., 1980; Donatelli and Greenfield., 1987; Vermeulen et al., 2000; Karduna et al., 2005; Vermeulen et al., 2006).

The classification of this mobilization is largely qualitative, and scientific evidence supporting the efficacy of this treatment approach is mixed (Bulgen et al., 1984; Green et al., 2003; Brudvig et al., 2011). The possibility of other joint treatment positions having greater clinical impact still exist and have not been ruled out. New, more effective joint treatment orientations could possibly be defined more thoroughly if therapy techniques could be further quantified.

No biomechanical study investigating the effects of commonly used gliding techniques on $\mathrm{GH}$ joint three dimensional (3D) biomechanics are currently known (Nicholson, 1985; Hsu et al., 2000; Hsu et al., 2000; Hsu and Headman., 2002; Hsu et al., 2002; Karduna et al., 2005; Johnson et al., 2007; Lin et al., 2008; Ho et al. 2009; Hsu et al., 2009; Kelley et al., 2009; McQuade et al., 2012). The objectives of this study were to develop an in vitro protocol to determine the 3D biomechanical effects of joint mobilization on the GH joint, and to then simulate these mobilizations.

Background information on the protocol development of design is explained in Chapter 2 of this thesis along with relevant anatomy descriptions. A protocol was first designed to capture the biomechanical responses to PT manual therapy on the GH joint by tracking the forces and displacements applied to the joint. From this data, 3D force and displacement end limits were defined for each of the tested manual therapies. Individual PT technique was also analyzed and contrasted against other therapists. This procedure is described in detail in Chapter 3. The force and displacement end limits were then used to develop a biorobotic testing protocol that could simulate GH joint gliding. $\mathrm{GH}$ gliding was then simulated on a specimen in a bioroboic testing platform using the forces and displacement limits defined by practiced PTs. The robotic glide simulation protocol is described in Chapter 4. 


\title{
CHAPTER 2. BACKGROUND OF THE GLENOHUMERAL JOINT
}

The human shoulder rests posteriorly, above the rib cage. Compared with other joints, the GH joint has a large ROM. This mobility introduces instability, making the shoulder one of the most injury-prone joints in the human body. To compensate for this instability, the shoulder joint is encompassed in numerous muscles and ligaments for reinforcement.

\begin{abstract}
Anatomy
Muscles connecting the scapula to the humerus make up a group of muscles called the rotator cuff. This 'cuff' wraps densely around the head of the humerus and is made up of four separate muscles: the subscapularis, supraspinatus, infraspinatus, and teres minor. These four muscles are the primary stabilizers of the glenohumeral joint. An anterior view of the shoulder is shown in Figure 2-1. A complete list of all major muscles directly involved in the shoulder joint along with their function is shown in Table 2-1.

The bones of the human shoulder are the clavicle, the scapula, and the humerus. These three bones make up three separate joints of the shoulder: the glenohumeral (primary joint of the shoulder), the acromioclavicular, and the sternoclavicular. The acromioclavicular joint is the junction between the acromion (located on the scapula) and the clavicle). This joint allows for the arm to be raised above the head. The glenohumeral joint is the primary joint of the shoulder; this joint is the junction between the humerus and the clavicle. Figure 2-1 shows bone and ligament layout.

The glenoid cavity holds the humeral head in place. The glenoid labrum is a fibro-cartilaginous structure that lines the cavity of the glenoid. This labrum deepens the socket, which increases the static stability of the glenohumeral joint. The long head of the biceps attaches to the superior end of the glenoid labrum. These structures are shown in Figure 2-2.
\end{abstract}

\section{Physiology}

Functionally, the GH joint is a 'ball and socket' system that relies heavily on muscles for stabilization. Figure 2-3 shows a more detailed picture of the densely packed ball socket with various ligaments, tendons, and various bone structures. Because of the shallow nature of the anatomy, this joint has all three translational DOFs. The shallow socket enables the joint to achieve an incredible range of motion compared to other joints. These three rotational DOFs in combination with the other three translational DOFs make this joint fully unconstrained. 
9 Acromial end of clavicle

10 Acromioclavicular joint

11 Acromion

12 Tendon of supraspinatus muscle (attached to the articular capsule)

13 Coraco-acromial ligament

14 Tendon of long head of biceps brachii muscle

15 Tendon of subscapularis muscle (attached to the articular capsule)

16 Intertubercular sulcus

17 Articular capsule of shoulder joint

18 Humerus

19 Trapezoid ligament

20 Coracoid process

21 Glenoid labrum

22 Shoulder joint (joint cavity)

23 Scapula

24 Supraspinatus muscle

25 Cartilage of glenoid cavity

26 Tendon of long head of triceps brachii muscle

27 Head of humerus (articular cartilage)

28 Epiphyseal line
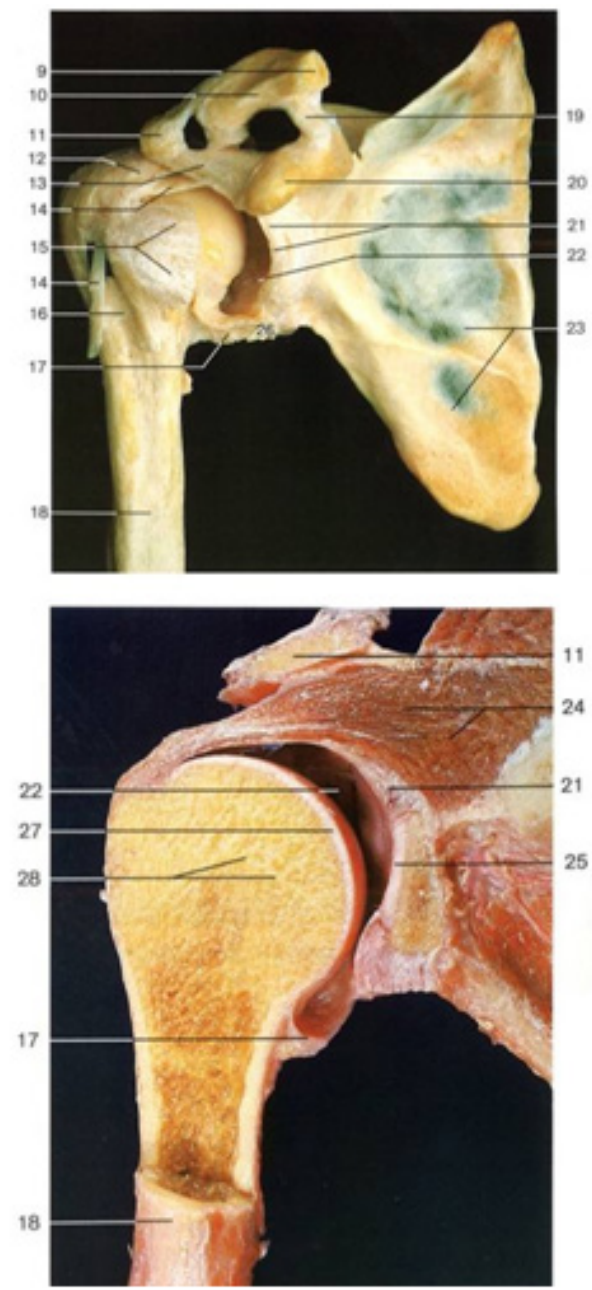

\section{Figure 2-1. Right GH joint.}

Upper: Anterior view of the shoulder. Part of the articular capsule has been removed for viewing. Lower: Anterior view of the coronal section of the right shoulder joint. Adapted with permission. Rohen, J., Yokochi C. Color atlas of anatomy : a photographic study of the human body. Baltimore, MD, Lippincott Williams \& Wilkins (2002), p. 356. (http://lww.com). 
Table 2-1. List of muscles involved in shoulder motion.*

\begin{tabular}{|c|c|c|}
\hline Muscle Name & Connection & Purpose \\
\hline Supraspinatus & $\begin{array}{l}\text { Rotator cuff muscles } \\
\text { connect the scapula to the } \\
\text { humerus }\end{array}$ & $\begin{array}{l}\text { Abducts the arm and is critical } \\
\text { in glenohumeral joint } \\
\text { movement and stability }\end{array}$ \\
\hline Infraspinatus & & $\begin{array}{l}\text { Rotates the arm externally and } \\
\text { is critical in glenohumeral joint } \\
\text { movement and stability }\end{array}$ \\
\hline Teres minor & & $\begin{array}{l}\text { Rotates the arm externally and } \\
\text { is critical in glenohumeral joint } \\
\text { movement and stability }\end{array}$ \\
\hline Subscapularis & & $\begin{array}{l}\text { Rotates the humerus internally } \\
\text { and is critical in glenohumeral } \\
\text { joint movement and stability }\end{array}$ \\
\hline $\begin{array}{l}\text { Deltoid, anterior } \\
\text { fibers }\end{array}$ & $\begin{array}{l}\text { Arises from the anterior } \\
\text { border and upper surface of } \\
\text { the lateral third of the } \\
\text { clavicle. }\end{array}$ & $\begin{array}{l}\text { Involved in shoulder abduction } \\
\text { when the shoulder is externally } \\
\text { rotated. The anterior deltoid is } \\
\text { weak in strict transverse flexion } \\
\text { but assists the pectoralis major } \\
\text { during shoulder transverse } \\
\text { flexion / shoulder flexion. }\end{array}$ \\
\hline $\begin{array}{l}\text { Deltoid, middle } \\
\text { fibers }\end{array}$ & $\begin{array}{l}\text { Arises from the lateral } \\
\text { margin and upper surface of } \\
\text { the acromion. }\end{array}$ & $\begin{array}{l}\text { Involved in shoulder abduction } \\
\text { when the shoulder is internally } \\
\text { rotated, shoulder flexion when } \\
\text { the shoulder is internally } \\
\text { rotated, and shoulder transverse } \\
\text { abduction (shoulder externally } \\
\text { rotated) -- but are not utilized } \\
\text { significantly during strict } \\
\text { transverse extension (shoulder } \\
\text { internally rotated). }\end{array}$ \\
\hline $\begin{array}{l}\text { Deltoid, posterior } \\
\text { fibers }\end{array}$ & $\begin{array}{l}\text { Arises from the lower lip of } \\
\text { the posterior border of the } \\
\text { spine of the scapula, as far } \\
\text { back as the triangular surface } \\
\text { at its medial end. }\end{array}$ & $\begin{array}{l}\text { Strongly involved in transverse } \\
\text { extension particularly since the } \\
\text { latissimus dorsi muscle is very } \\
\text { weak in strict transverse } \\
\text { extension. The posterior deltoid } \\
\text { is also the primary shoulder } \\
\text { hyperextensor. }\end{array}$ \\
\hline Serratus anterior & $\begin{array}{l}\text { Originates on the surface of } \\
\text { the upper eight ribs at the } \\
\text { side of the chest and inserts } \\
\text { along the entire anterior } \\
\text { length of the medial border } \\
\text { of the scapula. }\end{array}$ & $\begin{array}{l}\text { It fixes the scapula into the } \\
\text { thoracic wall and aids in } \\
\text { rotation and abduction of the } \\
\text { shoulders. }\end{array}$ \\
\hline
\end{tabular}


Table 2-1. (Continued).

\begin{tabular}{|c|c|c|}
\hline Muscle Name & Connection & Purpose \\
\hline Subclavius & $\begin{array}{l}\text { Located inferior to the } \\
\text { clavicle, originating on the } \\
\text { first rib and inserting on the } \\
\text { subclavian groove of the } \\
\text { clavicle. }\end{array}$ & $\begin{array}{l}\text { It depresses the lateral clavicle } \\
\text { and also acts to stabilize the } \\
\text { clavicle. }\end{array}$ \\
\hline Pectoralis minor & $\begin{array}{l}\text { Arises from the third, fourth, } \\
\text { and fifth ribs, near their } \\
\text { cartilage and inserts into the } \\
\text { medial border and upper } \\
\text { surface of the coracoid } \\
\text { process of the scapula. }\end{array}$ & $\begin{array}{l}\text { This muscle aids in respiration, } \\
\text { medially rotates the scapula, } \\
\text { protracts the scapula, and also } \\
\text { draws the scapula inferiorly. }\end{array}$ \\
\hline Sternocleidomastoid & $\begin{array}{l}\text { Attaches to the sternum } \\
\text { (sterno-), the clavicle (cleido- } \\
\text { ), and the mastoid process of } \\
\text { the temporal bone of the } \\
\text { skull. }\end{array}$ & $\begin{array}{l}\text { Most of its actions flex and } \\
\text { rotate the head. In regards to } \\
\text { the shoulder, however, it also } \\
\text { aids in respiration by elevating } \\
\text { the sternoclavicular joint when } \\
\text { the head is fixed. }\end{array}$ \\
\hline Levator scapulae & $\begin{array}{l}\text { Arises from the transverse } \\
\text { processes of the first four } \\
\text { cervical vertebrae and inserts } \\
\text { into the medial border of the } \\
\text { scapula. }\end{array}$ & $\begin{array}{l}\text { It is capable of rotating the } \\
\text { scapula downward and } \\
\text { elevating the scapula. }\end{array}$ \\
\hline $\begin{array}{l}\text { Rhomboid major } \\
\text { and minor }\end{array}$ & $\begin{array}{l}\text { They arise from the spinous } \\
\text { processes of the thoracic } \\
\text { vertebrae t1 to t5 as well as } \\
\text { from the spinous processes of } \\
\text { the seventh cervical. They } \\
\text { insert on the medial border of } \\
\text { the scapula, from about the } \\
\text { level of the scapular spine to } \\
\text { the scapula's inferior angle. }\end{array}$ & $\begin{array}{l}\text { They are responsible for } \\
\text { downward rotation of the } \\
\text { scapula with the levator } \\
\text { scapulae, as well as adduction } \\
\text { of the scapula. }\end{array}$ \\
\hline Trapezius & $\begin{array}{l}\text { Arises from the occipital } \\
\text { bone, the ligamentum } \\
\text { nuchae, the spinous process } \\
\text { of the seventh cervical, and } \\
\text { the spinous processes of the } \\
\text { thoracic vertebrae, and from } \\
\text { the corresponding portion of } \\
\text { the supraspinal ligament. It } \\
\text { inserts on the lateral clavicle, } \\
\text { the acromion process, and } \\
\text { into the spine of the scapula. }\end{array}$ & $\begin{array}{l}\text { Different portions of the fibers } \\
\text { perform different actions on } \\
\text { the scapula: depression, } \\
\text { upward rotation, elevation, and } \\
\text { adductions. }\end{array}$ \\
\hline
\end{tabular}

*Gray, H, Pickering, P.T, Howden R. Gray's Anatomy. Philadelphia: Courage (1974). 

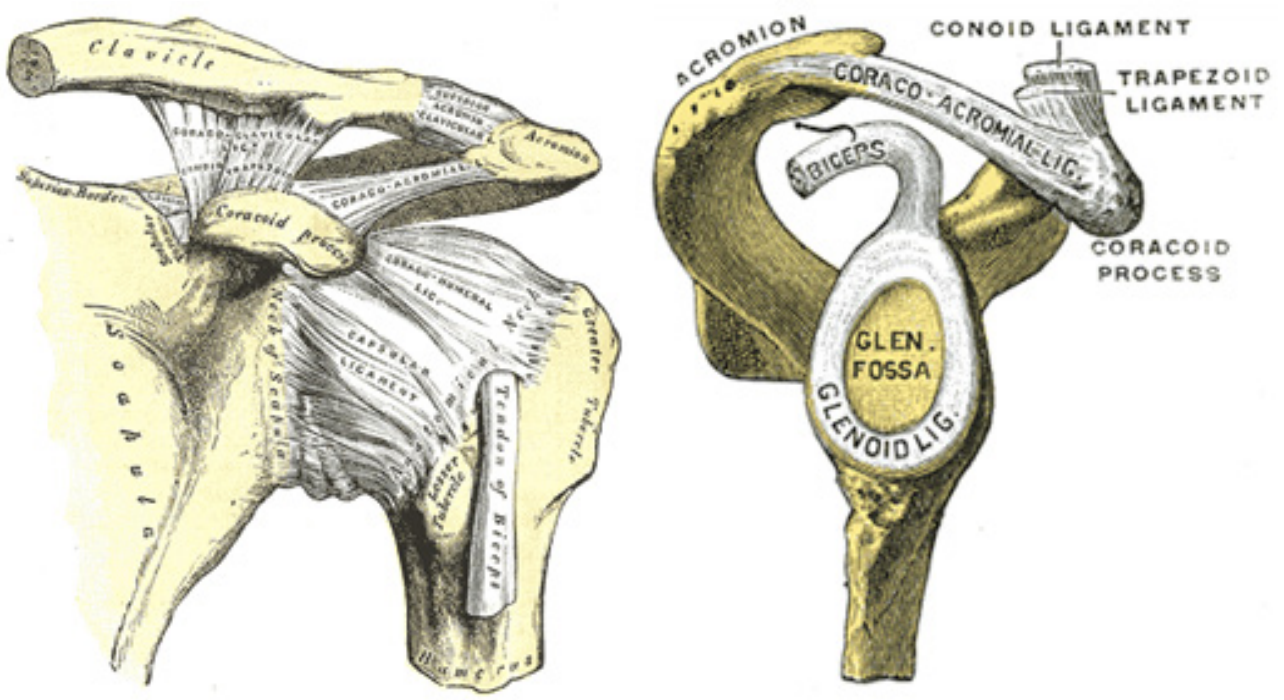

Figure 2-2. Sketch of the GH joint and scapula.

Left: left glenohumeral joint with labeled tendons and ligaments. Right: right scapula emphasizing the glenoid fossa and glenoid ligament.

Reprinted with permission. Gray, H, Pickering, Pick T, Howden R. Gray's Anatomy. Philadelphia: Courage (1974), p. 501. 

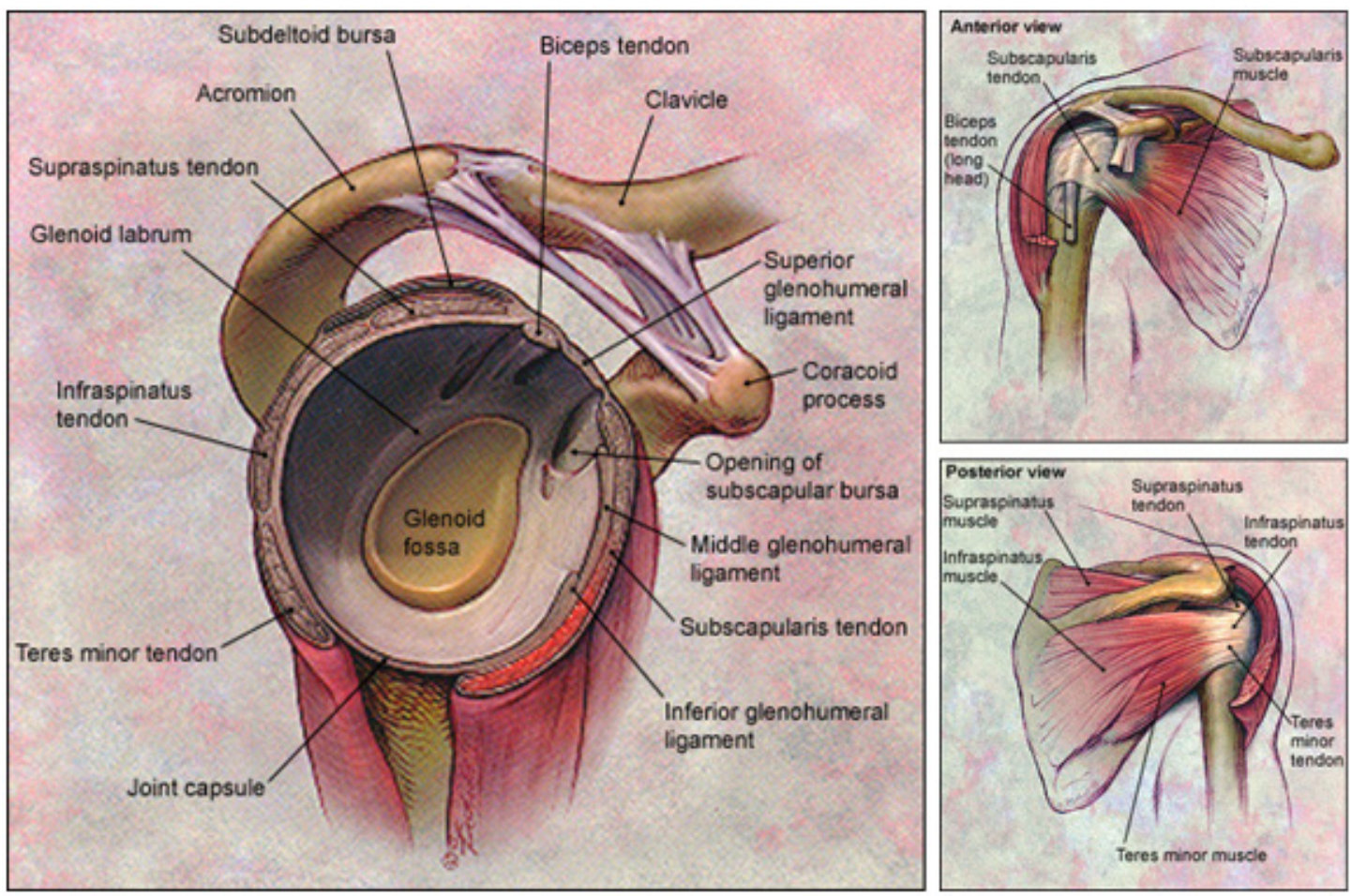

Figure 2-3. Lateral view of the right shoulder GH joint.

Humerus has been removed.

Photographs adapted with permission. Fongemie, A., Buss, D., and Rolnick, S.

"Management of Shoulder Impingement Syndrome and Rotator Cuff Tears." American Family Physician (1998), p. 667. 


\section{Consideration of Testing Methodologies}

Two types of systems are used for fresh cadaveric testing: Open Loop (or "open chain") (Wuelker and Wirth, 1995) and Closed Loop (or "closed chain") (Bryce et al., 2010) systems. Closed loop systems (as seen in Figure 2-4) have a rigid connection at the other end of the joint being studied. Open loop systems (as seen in Figure 2-5) have an open end to the test apparatus. The arm is hanging off of the end with no other connection. Both systems have advantages and disadvantages: This particular open loop system requires manual articulation of the joint; the closed loop system has more capacity when it comes to imposing different loading simulations on the joint. Because the glenohumeral joint contains only two bones, the forces captured are the exact forces that the glenoid sees (in other words, the closed loop setup allows for a direct force reading of the glenohumeral joint).

Closed system can simulate the same motions of the open loop, but the closed loop can take the simulation a step farther. Because the joint is monitored by the 6 axis load cell (which can be programmed to apply a specified force vector on the joint), the closed loop system can induce loading simulations (picking up a cup of coffee, standing up out of a chair, ect.) along with motion responses. The disadvantage of the closed loop system is the inability to see motion responses directly. These responses are seen as force buildup, though. Using force control, motion responses can be found using the closed loop system.

The closed loop system was used to model the protocol development with the UTHSC Biorobotic Laboratory. The RTP's gantry setup coupled with the closed loop configuration allows for full exploration of internal/external rotational limits throughout simulated motion. Allowing the humerus to be mounted into the robot gimbal causes the specimen to be mounted inverted. This appears cumbersome at first, until rotator cuff loads are considered. Because the robot's 4DOF do not include saggital abduction motion, the scapula was mounted rigidly onto a 1DOF table. Simulated muscle forces can then be applied directly to the joint. All forces applied through the scapula are seen by the upper load cell because the glenohumeral joint is a 2-body (single joint) system.

\section{Pre-testing Preparation}

Prior to testing, radiographs were taken of the specimens to check for anatomical abnormalities and to calculate physical distances from the approximate joint center to the potted ends. Appendix A contains radiographs of each specimen along with figures of each specimen mounted in testing apparatus with force frame offsets.

\section{Robot Platform Upgrades}

The existing robotic platform was heavily modified in order to increase the simulation capacity of the system. Rotary actuators were upgraded from a $10 \mathrm{Nm}$ torque 


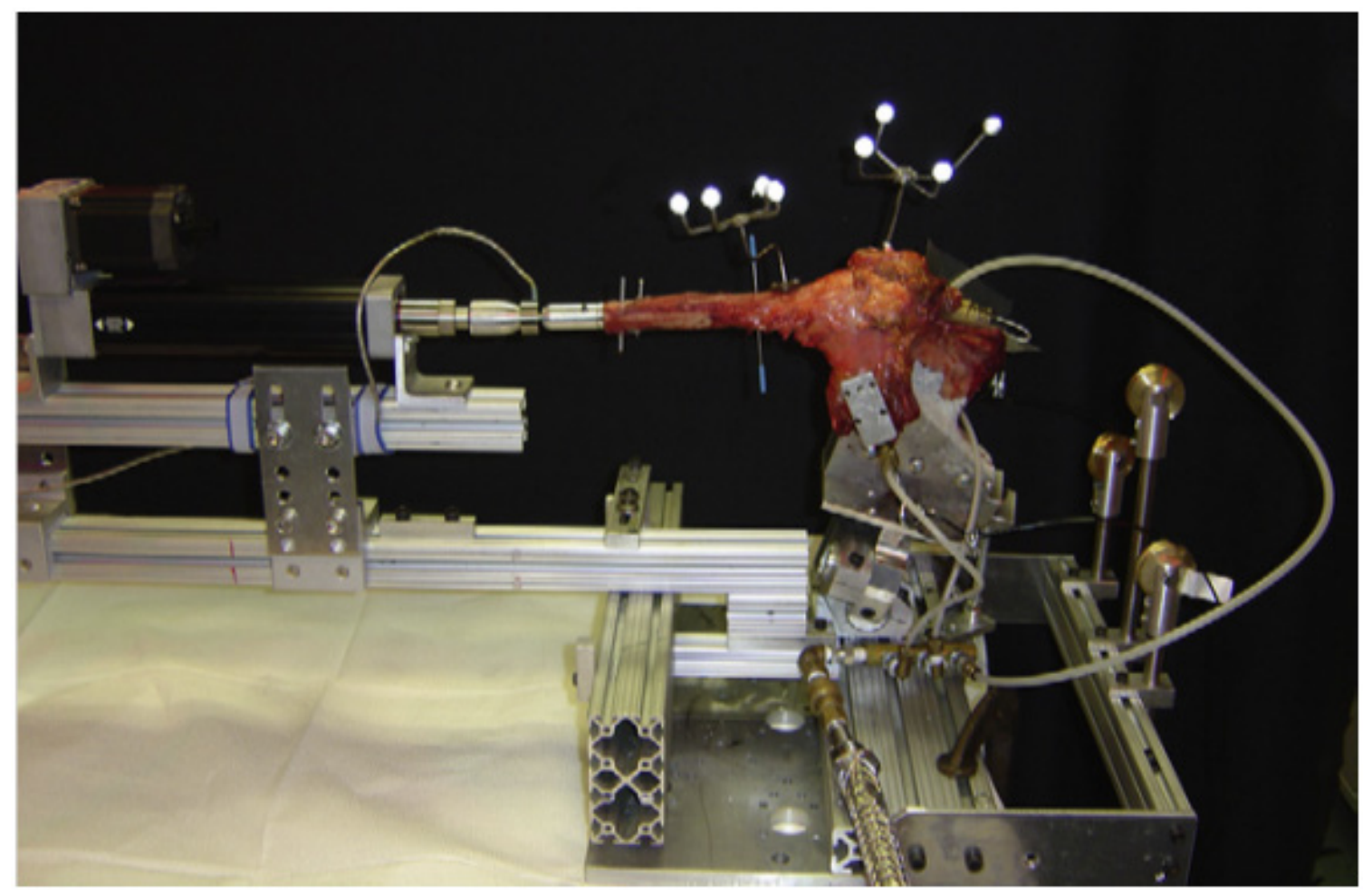

Figure 2-4. Example of a closed loop testing apparatus.

Reprinted with permission. Bryce, C.D., Davison, A.C., Okita, N., Lewis, G.S., Sharkey, N.A., and Armstrong, A.D. "A Biomechanical Study of Posterior Glenoid Bone Loss and Humeral Head Translation." Journal of Shoulder and Elbow Surgery (2010), p. 995. 


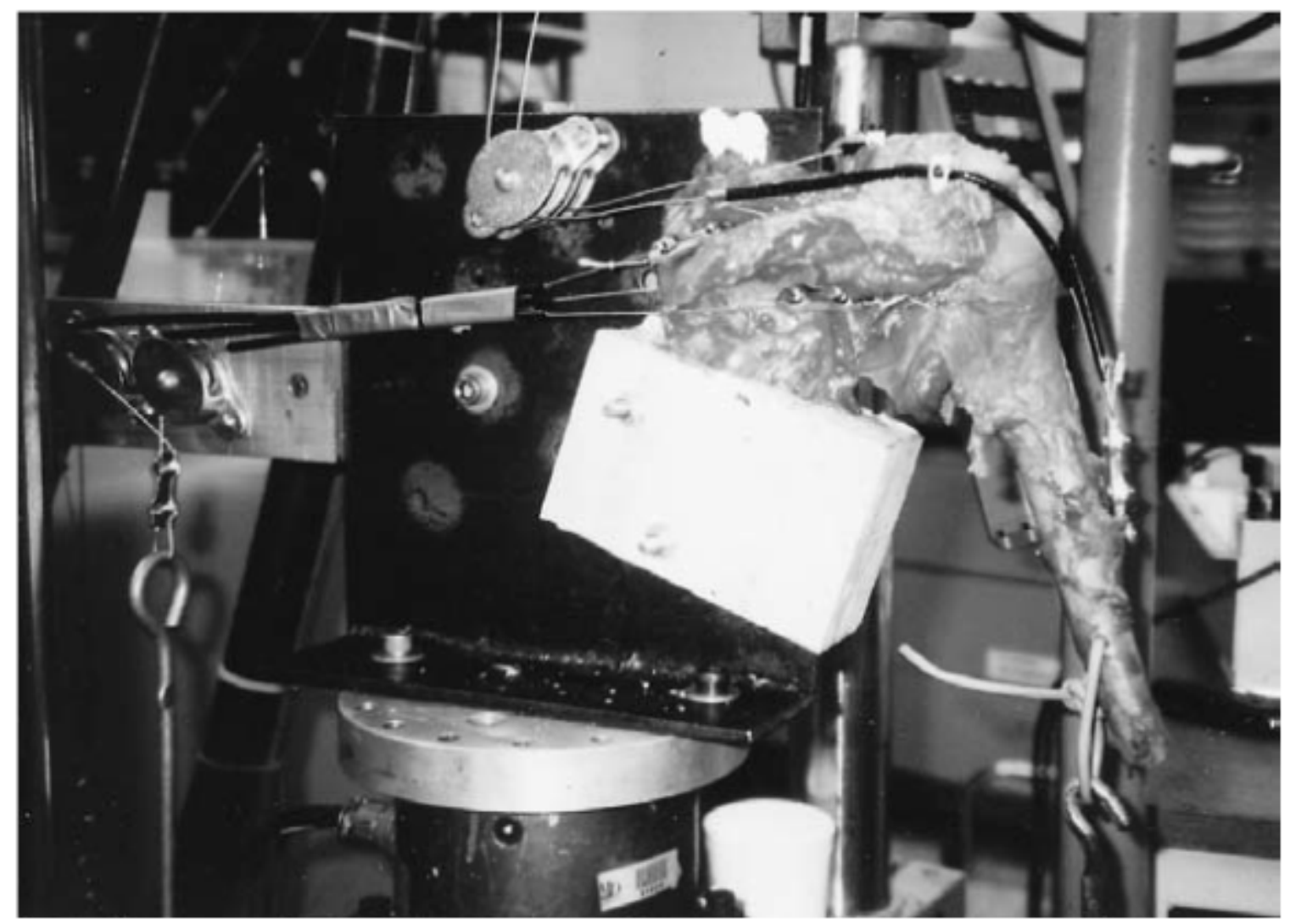

Figure 2-5. Pictures of an open loop testing apparatus.

The specimen is rigidly attached to a base plate load cell with the humerus hanging freely in a neutral, upright position. Rotator cuff muscles are being simulated by loaded sutures into each individual muscle.

Reprinted with permission. Bono, C.M., Renard, R., and Levy, A.S. "Effect of Displacement of Fractures of the Greater Tuberosity on the Mechanics of the Shoulder." The Journal of Bone and Joint Surgery (2001), p. 1057. 
capacity motor (Kollmorgen $9 \mathrm{FG}$ ) to a $80 \mathrm{Nm}$ capacity motor (Harmonic Drive FHA$25 \mathrm{C}-160)$. This new motor upgrade increased the resolution up from $0.0045^{\circ}$ to

$0.000225^{\circ}$. Figure 2-6 shows the new robotic system. An extra motor was installed on the robot; this extra motor (also a Harmonic Drive FHA-25C-160), named the 'muscle motor', was installed so that future projects could utilize it for simulating muscle forces in dynamic joint simulations. 


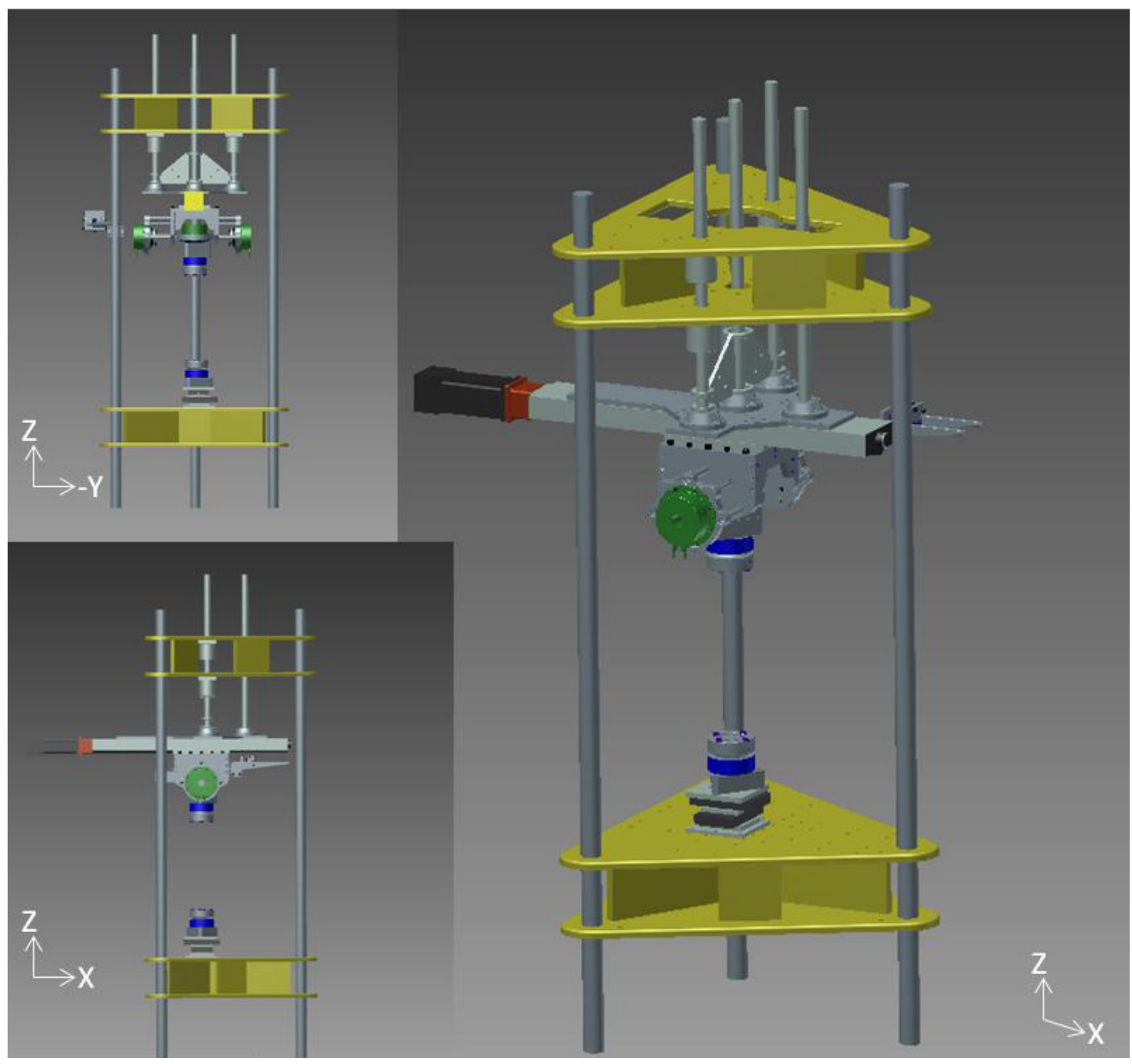

Figure 2-6. Computer aided drawing of the robotic testing platform. Upgraded rotary muscles have been installed. 


\section{CHAPTER 3. IN VITRO MANUAL THERAPY OF THE GLENOHUMERAL JOINT}

Chapter 3 encompasses the results of an initial Physical Therapist manual therapy experiment. The purpose of this study was to investigate the forces applied by therapists during a gliding treatment session. This chapter was formed into a manscript for submission to the journal 'Manual Therapy'.

\section{Introduction}

Shoulder dysfunction is the second most common reason for referral to outpatient physical therapy (Vermeulen et al., 2000). Glenohumeral joint hypomobility can contribute directly (adhesive capsulitis) or indirectly (shoulder impingement syndrome) to shoulder pain and immobility (Kelley et al., 2009; Wilk et al., 2009; Brudvig et al., 2011). Physical therapists (PTs) use joint mobilization techniques as the intervention of choice to address capsular hypomobility (Maitland, 1977; Rollins et al., 1980; Donatelli and Greenfield., 1987; Vermeulen et al., 2000; Karduna et al., 2005; Vermeulen et al., 2006). Manual shoulder joint mobilizations are often incorporated to restore restricted arthrokinematic motions that can restrict osteokinematic motion. Increasing joint play motions prior to cardinal plane stretching is thought to be a safer and less painful method of increasing joint range of motion (Nicholson, 1985; Vermeulen et al., 2000; Vermeulen et al., 2006; Johnson et al., 2007; Wilk et al., 2009). Scientific evidence supporting the efficacy of this treatment approach is mixed (Bulgen et al., 1984; Green et al., 2003; Brudvig et al., 2011).

Kelley et al proposed a model to guide rehabilitation for individuals with adhesive capsulitis. It includes classifying patients into levels of irritability (high/moderate/low) and suggests using specific manual mobilization techniques dependent upon the irritability level. This model is supported by previous research which found shoulder joint mobilization to be effective for decreasing pain (Johnson et al., 2007; McQuade et al., 2012) and increasing range of motion (Nicholson, 1985; Vermeulen et al., 2000; Vermeulen et al., 2006; Johnson et al., 2007) in patients with shoulder joint hypomobility. Cadaveric studies have demonstrated increased range of motion following application of joint mobilization techniques (Branch et al., 1999; Hsu et al., 2000; Lin et al., 2008). However, many intervention studies have found no improvement in ROM following joint mobilization. Bulgen (Bulgen et al., 1984) compared the effects of three individual treatments (glucocorticosteroid injection, joint mobilization, and ice) to a control group and found little long-term advantage for any intervention. Only steroid injections were shown to benefit pain and range of motion, and this effect was only in the early stages. A 2003 systematic review (Green et al., 2003) concluded that mobilization with exercise is of greater benefit than exercise alone to improve both pain and range of motion. However, the treatment effect was small and did not apply to individuals with a diagnosis of adhesive capsulitis. Brudvig et al (2011) performed a meta-analysis that included seven intervention studies and determined that the current evidence is 
inconclusive with regard to the benefit of joint mobilization with exercise compared to exercise alone. A recent prospective, randomized, controlled study supports this conclusion, determining that providing mobilization in addition to advice and exercise did not improve pain, range of motion or impairment rating when compared to advice and exercise alone (Wilk et al., 2009).

It is clear that although it is common clinical practice to perform joint mobilization, the efficacy has not been established. In conducting a review of the literature, we noted many differences in the application of joint mobilization across the studies. Specifically different intensities of mobilization were performed, for different durations, and with different frequency. Few studies have even explored whether different operators (therapists) would demonstrate inter-operator consistency for force applied or translation achieved when performing a particular grade of mobilization (Hsu and Headman., 2002; Hsu et al., 2002).

Physical therapists mobilize joints by applying displacements and forces to increase joint range of motion (ROM) and reduce pain. (Hertling and Kessler., 2006) The level of mobilization is typically based on a five grade scale system (Figure 3-1) (Rollins et al., 1980). However, characterizing the amount of mobilization using this model remains largely qualitative.

When treating a patient with GH joint hypomobility, the PT positions the joint appropriately (e.g., neutral, resting, or end range) and performs a mobilization glide along a specific axis or direction. The goal is to translate the joint along the mobilization axis without inducing any rotation of the humeral head. As the joint displacement increases the surrounding tissue structures are engaged and the amount of force needed to continue the displacement will increase. The rate of increase in force resistance is a function of the structural stiffness properties of the GH joint. Quantifiable measure of the applied forces and displacement directions would help to define the structural stiffness properties of the joint which could further be used to differentiate between the types of mobilizations techniques and better understand their biomechanical impact.

Hsu et al (2009) investigated the effects of axial distraction mobilizations of the GH joint in the neutral and end range positions in a cadaveric model. Mobilization was gained at the end range of GH abduction. They suggested that the forces and displacements applied by the therapists were dependent on the GH joint position. This study only addressed the general response of the PT population and did not compare between individual therapists. Distractive forces up to $72 \mathrm{~N}$ were applied to the $\mathrm{GH}$ joint, but the standard deviation of the forces applied by the different therapists was $20 \mathrm{~N}$ in every testing position. This outcome suggests that the therapists were not consistent between one another in how the forces were applied. Further, the consistency in how the different Physical Therapists performed similar mobilization techniques remains unknown. 
Treatments are categorized by 5 grades:

Pain Treatment:

- Grade I: Low amplitude performed in middle of joint

- Grade II: High amplitude performed in the middle of the joint Increase Range of Motion:

- Grade III:

High amplitude performed at end range of joint

- Grade IV:

Low amplitude performed at end range of joint

- Grade V:

Low amplitude/quick thrust at end range of joint

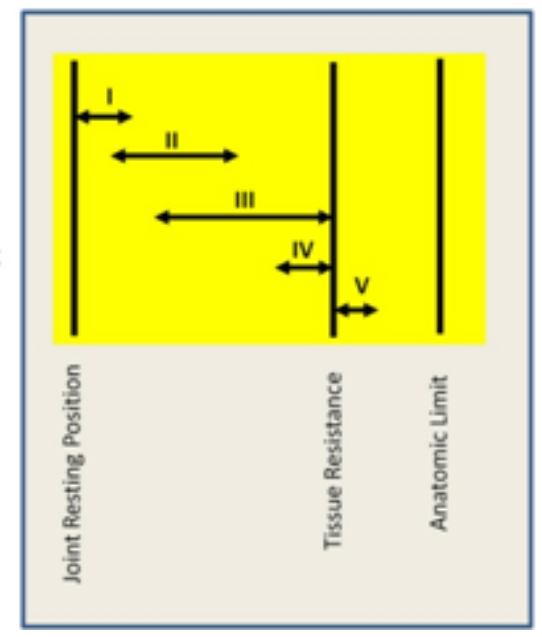

Figure 3-1. Grades of mobilization.

Source: Maitland G. Peripheral Manipulation. London: Butterworths. (1977). 
No other biomechanical studies investigating the effects of commonly used gliding techniques on $\mathrm{GH}$ joint 3D biomechanics are currently known (Nicholson, 1985; Hsu et al., 2000; Hsu et al., 2000; Hsu and Headman., 2002; Hsu et al., 2002; Karduna et al., 2005; Johnson et al., 2007; Lin et al., 2008; Ho et al. 2009; Hsu et al., 2009; Kelley et al., 2009; McQuade et al., 2012). The purpose of this study was to develop a biomechanical model for quantifying $\mathrm{GH}$ joint mobilization techniques performed by experienced Physical Therapists. Anterior, posterior, and inferior glides were investigated in two GH positions using grade IV non-oscillatory mobilizations.

\section{Methods}

\section{Tissue Preparation}

One right human cadaveric shoulder (Male, Age: 52 years) was procured and prepared for testing. All superficial soft tissue was removed until the RC muscles and tendons were exposed. Monofilament nylon was sutured to the $\mathrm{RC}$ tendons of the subscapularis, supraspinatus, and teres minor/infraspinatus using a modified locking suture technique (Krackow et al., 2011). The teres minor and infraspinatus share a similar magnitude; both were combined into a single muscle line for simplicity. The inferior angle of the scapula was then potted in a low melting point bismuth alloy (Figure 3-2) with the scapula in a neutral upright orientation. The humerus was cut $250 \mathrm{~mm}$ from the joint center and approximately $10 \mathrm{~N}$ of a low melting point bismuth alloy was added to the end of the humeral shaft. The resultant combined weight of the humeral bone and fixture weight was $15 \mathrm{~N}$. The prepared specimen was then stored in a freezer at $-10^{\circ} \mathrm{F}$ and thawed at $40^{\circ} \mathrm{F} 24$ hours prior to testing.

\section{In Vitro Testing Model}

The specimen was rigidly mounted to a 6-axis load cell that was part of an existing biomechanical testing platform (Kelly and DiAngelo., 2006). The scapula was oriented in an upright neutral position and the humerus freely suspended, as shown in Figure 3-3. The anatomical coordinate system was aligned with the load cell's coordinate system where $+Z$ load cell axis was aligned with the anterior anatomical axis, $-Z$ load cell axis aligned with the posterior anatomical axis, and $-Y$ load cell axis aligned with the inferior anatomical axis. Custom fixtures were used to apply RC forces along the line of action of the rotator cuff muscles and set to $5 \mathrm{~N}$ each; an amount determined by an experienced PT to reproduce a physiologically equivalent response. In the end, the overall action of the RC muscle forces was to pull the humerus medially into the scapula without introducing any significant rotational bending moment about the GH joint center. This condition was confirmed by transforming the output from the six axis load cell to the center of the $\mathrm{GH}$ joint and analyzing the joint mechanics (i.e., primarily compressive joint loading state with minimal rotational bending moment). To further assist in the 


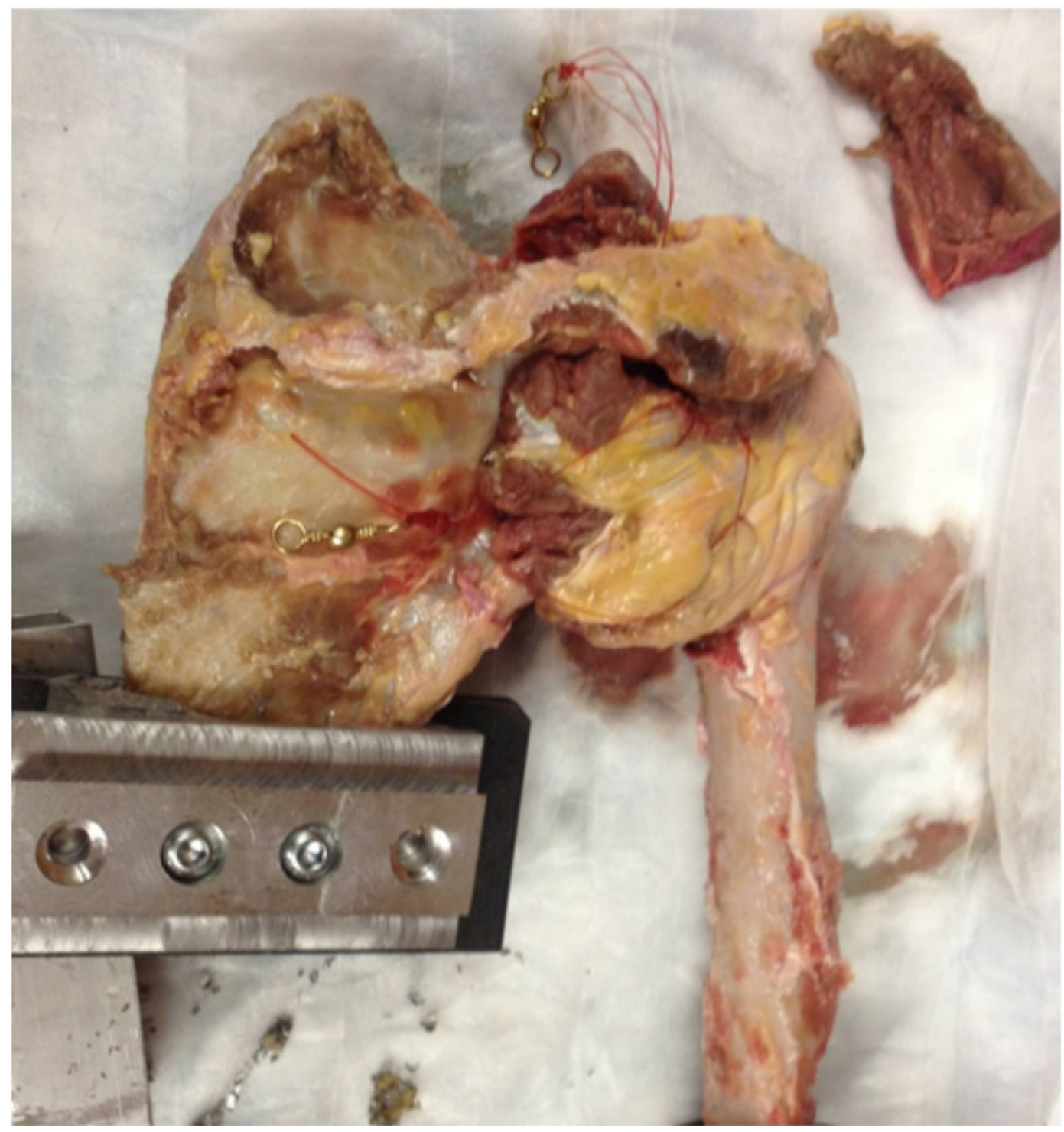

Figure 3-2. Sutured and potted specimen. 


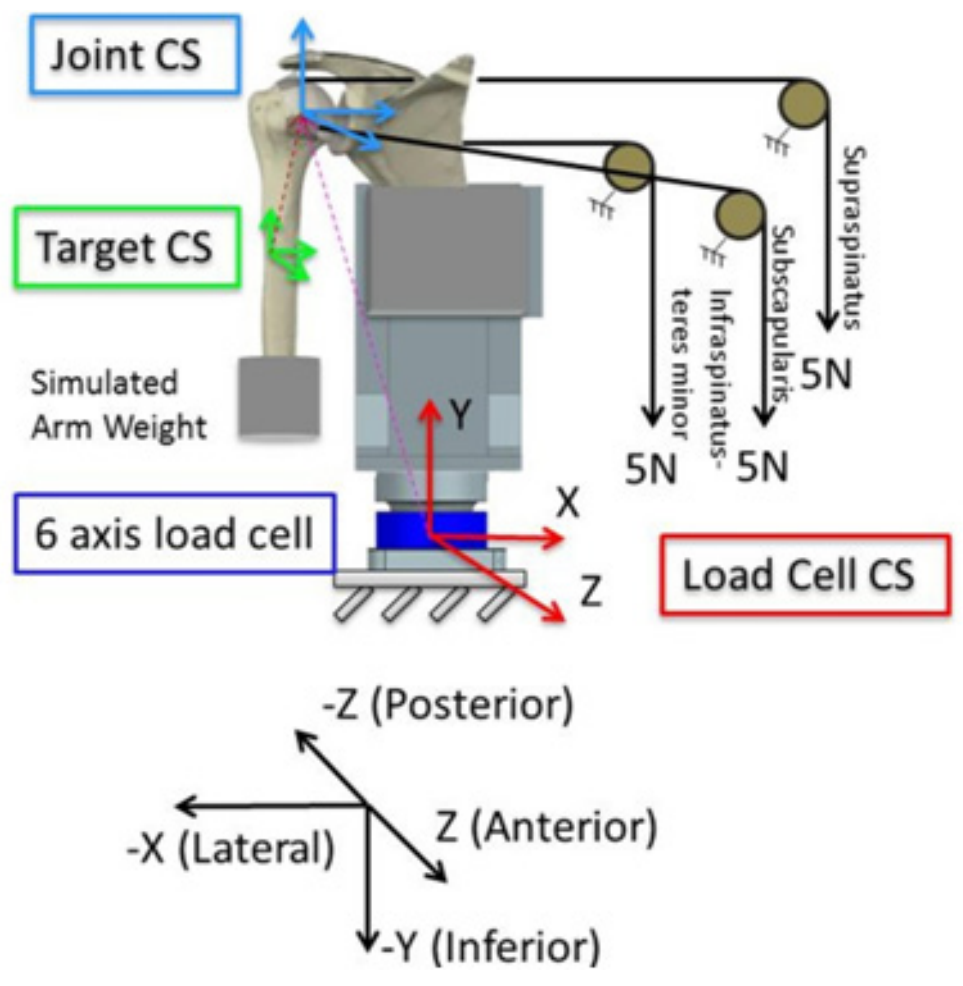

Figure 3-3. Schematic of coordinate system transformations.

Load cell coordinate system (CS) and 3D target CS were both transferred to the GH joint center. The camera CS frame was aligned with the anatomical reference frame of the specimen. 
mechanics analysis, a new baseline loading condition was established by zeroing the forces and moments after the RC loads were applied. A pair of three dimensional (3D) target arrays was rigidly attached to the humerus and scapula for motion analysis. The coordinates of the camera targets systems were also transformed to the GH joint center and analyzed with respect to the GH joint center.

Two Physical Therapists, with a combined working experience in excess of 50 years, were instructed to perform three grade IV non-oscillatory GH mobilization glides without consultation. Three glide directions were analyzed: posterior (P), anterior (A), and inferior (I) along with two GH joint positions: Neutral and Resting positions. The different glide directions and GH joint positions are described in Figure 3-4.

\section{Data Management}

Individual components of the 3D forces were collected at $20 \mathrm{~Hz}$ while the 3D motion of the humerus relative to the scapula was sampled at $10 \mathrm{~Hz}$. Motion and forces were transformed to the GH joint center and aligned with the anatomical axes. The resultant mobilization axis forces and 3D forces were compared between PTs and arm positions using a two-way repeated measures ANOVA with Holm-Sidak post hoc analyses.

Additionally, the 3D GH joint stiffness values were calculated by dividing the net $3 \mathrm{D}$ force vector by the net $3 \mathrm{D}$ positional vector $(\mathrm{V})$, where the $3 \mathrm{D}$ force and displacement vectors were calculated where the magnitude was determined using the Pythagorean Theorem (i.e., $\left(\sqrt{X^{2}+Y^{2}+Z^{2}}=\sqrt{V^{2}}\right)$. The $3 \mathrm{D}$ stiffness values were compared using a paired t-test $(\mathrm{p}<0.05)$.

\section{Results}

\section{Validation of GH Joint Mechanics}

The initial rotational bending moments about the GH joint after the RC loads were applied were less than $0.02 \mathrm{Nm}$ confirming the initial role of the $\mathrm{RC}$ muscles was to stabilize the joint. During the mobilization glides, both PTs maintained flexion/extension, abduction/adduction and internal/external orientations of the humerus to within a few degrees (Table 3-1).

\section{Mobilization Axis Properties}

The individual force components along the mobilization axes are listed in

Table 3-2. The mean mobilization force values from the three glides performed by the 

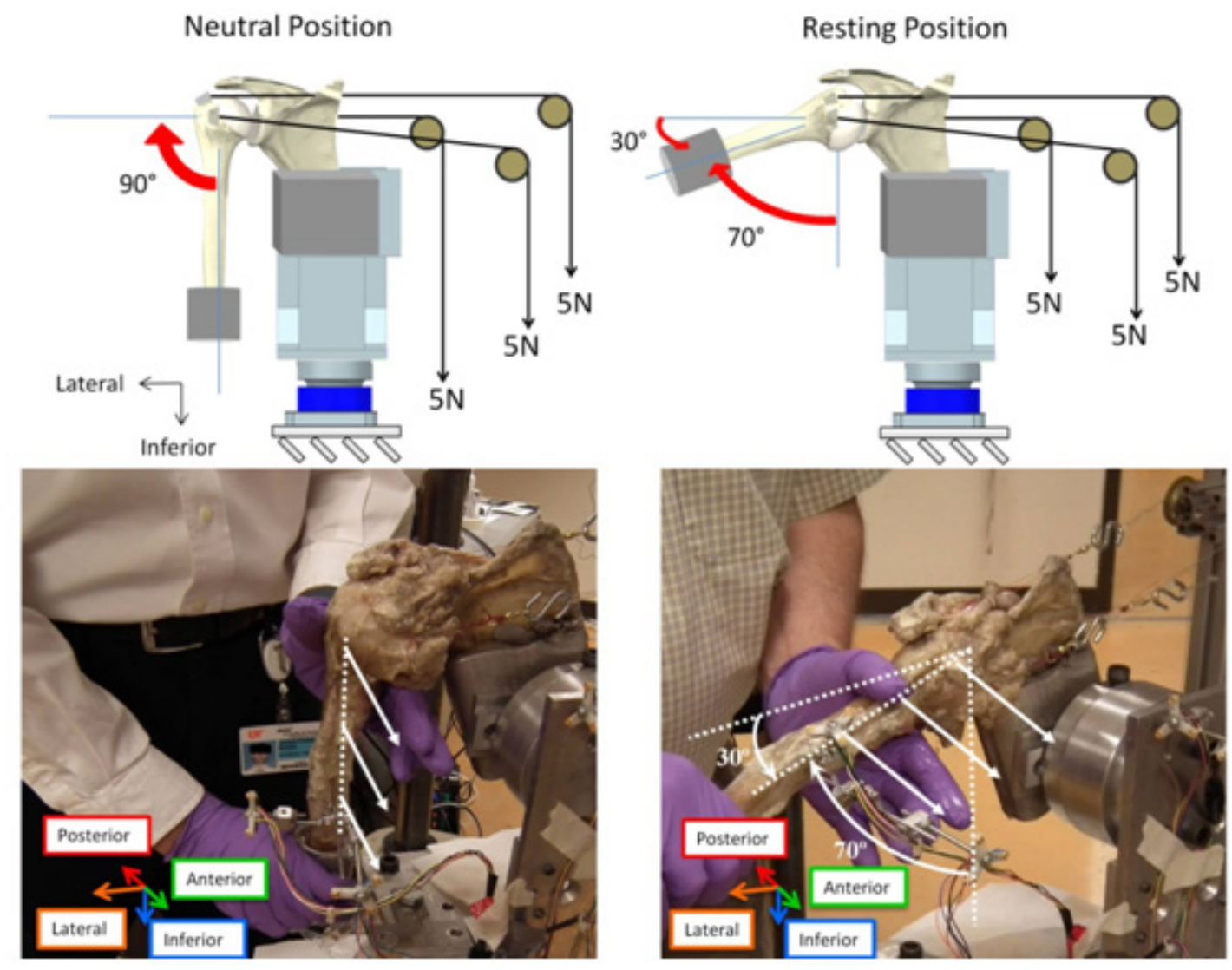

Figure 3-4. Joint positions of the GH joint.

Top: Demonstration of GH specimen in the neutral position. For the anterior glide, the humerus axis was $90^{\circ}$ to the ground as in a standing upright position.

Bottom: Arrangement of GH specimen in the resting position. The humerus was abducted $70^{\circ}$ from a vertical neutral position and then rotated $30^{\circ}$ in horizontal adduction. For the anterior glide depicted here, the humerus was translated along the anterior axis only; no humeral rotation occurred. 
Table 3-1. Rotational standard deviation during glides (degrees).

\begin{tabular}{|c|c|c|c|c|c|c|c|c|c|c|c|c|}
\hline \multirow[b]{2}{*}{ Rotation } & \multicolumn{2}{|c|}{$\begin{array}{c}\text { Anterior } \\
\text { Rest }\end{array}$} & \multicolumn{2}{|c|}{$\begin{array}{c}\text { Anterior } \\
\text { Neutral }\end{array}$} & \multicolumn{2}{|c|}{$\begin{array}{c}\text { Posterior } \\
\text { Rest }\end{array}$} & \multicolumn{2}{|c|}{$\begin{array}{c}\text { Posterior } \\
\text { Neutral }\end{array}$} & \multicolumn{2}{|c|}{$\begin{array}{c}\text { Inferior } \\
\text { Rest }\end{array}$} & \multicolumn{2}{|c|}{$\begin{array}{l}\text { Inferior } \\
\text { Neutral }\end{array}$} \\
\hline & PT1 & PT2 & PT1 & PT2 & PT1 & PT2 & PT1 & PT2 & PT1 & PT2 & PT1 & PT2 \\
\hline $\boldsymbol{\theta X}$ & 3.3 & 1.3 & 1.4 & 1.4 & 3.4 & 0.9 & 1.4 & 1.3 & 2.1 & 1.3 & 1.0 & 1.6 \\
\hline$\theta \mathbf{Y}$ & 2.6 & 1.3 & 1.4 & 1.3 & 3.1 & 1.5 & 1.2 & 1.3 & 3.0 & 1.6 & 1.3 & 1.1 \\
\hline$\theta \mathbf{Z}$ & 1.1 & 1.1 & 2.0 & 0.9 & 2.4 & 1.1 & 1.7 & 1.4 & 3.3 & 1.8 & 1.1 & 1.5 \\
\hline
\end{tabular}

PT1: Physical Therapist 1, PT2: Physical Therapist 2, $\theta \mathrm{X}$ : Flexion/Extension, $\theta \mathrm{Y}$ : Internal/External Rotation, $\theta \mathrm{Z}$ : Abduction/Adduction. 
Table 3-2. Forces (N) along the mobilization axis.

\begin{tabular}{lcccccc}
\hline Glide & Therapist & Run 1 & Run 2 & Run 3 & Mean & SD \\
\hline Anterior Rest & PT1 & 16.7 & 21.2 & 22.4 & 20.1 & 3 \\
& PT2 & 19.6 & 18.3 & 18.7 & 18.9 & 0.6 \\
Anterior Neutral* & PT1 & 22.8 & 23.2 & 28.5 & 24.9 & 3.2 \\
\cline { 2 - 6 } & PT2 & 12.6 & 15.5 & 17.5 & 15.2 & 2.5 \\
Posterior Rest* & PT1 & -29.7 & -29.7 & -30.6 & -30 & 0.5 \\
& PT2 & -33.8 & -34.2 & -37.9 & -35.3 & 2.2 \\
Posterior Neutral & PT1 & -20 & -20 & -18.7 & -19.6 & 0.7 \\
\cline { 2 - 6 } Inferior Rest & PT2 & -23.2 & -20.4 & -24.4 & -22.7 & 2.1 \\
& PT1 & -28.3 & -24.3 & -31.6 & -28.1 & 3.7 \\
Inferior Neutral & PT2 & -24.3 & -27.9 & -26.3 & -26.2 & 1.8 \\
& PT1 & -27.5 & -27.5 & -27.1 & -27.4 & 0.2 \\
\hline & PT2 & -28.3 & -27.1 & -27.1 & -27.5 & 0.7 \\
\hline
\end{tabular}

N: Newton, PT1: Physical Therapist 1, PT2: Physical Therapist 2, SD: Standard Deviation.

*Signifies a statistically significant difference between therapists for $\mathrm{p}<0.05$. 
two Physical Therapists (PT1 and PT2) are shown in Figure 3-5. Mobilization forces were significantly different between the PTs for the anterior mobilization in the neutral position and the posterior mobilization in the resting position. Additionally the only statistically significant difference between neutral and resting GH positions occurred during the Posterior mobilization.

\section{D Mobilization Properties}

Although therapists mobilize joints along a particular direction, the structural properties of the joint as well as the orientation of the joint surfaces may not directly align with the mobilization directions. As such, some forces may build up along the nonmobilization axes. To study the biomechanical effects along those axes, the net 3D stiffness values and non-mobilization axis forces were further analyzed.

The mean values of the 3D forces, 3D displacements and resultant 3D stiffness values are listed in Table 3-3. This data was all recorded from an absolute coordinate system. The complete dataset is provided in Appendix B. In the 3D stiffness, the only significant difference between the PTs occurred during the posterior resting mobilization. The component forces from the posterior resting glide are shown in Table 3-4. A further analysis of the component forces associated with this mobilization was done to study the effects of the non-mobilization forces, in addition to those forces along the mobilization axis as seen in Figure 3-6. The three force components are shown in Figure 3-6B. A statistically significant difference occurred between the PTs in the amount of lateral force they applied to the GH joint during the posterior glide. One PT laterally distracted the joint throughout the posterior mobilization while the other applied minimal nonmobilization forces across the joint. When comparing between neutral and resting positions of the GH joint, significant differences occurred during the posterior glide for both PTs and during the anterior glide mobilization for PT1.

\section{Discussion}

The primary action of a mobilization is to apply traction/distraction to the joint along a linear axis (i.e., translation) and load the surrounding joint muscle and tissue structures in a controlled manner while preventing or minimizing joint rotations. In this study both PTs were able to control the orientation of the GH to within a few degrees while performing each mobilization. The ability to report the joint orientation during the mobilization could serve as a teaching tool for PT students to practice and learn how to mobilize the $\mathrm{GH}$ joint without rotating it.

Due to the soft tissue structures and shape of the articular joint surfaces, forces can build up along the non-mobilization axes. As such an analysis of the 3D force and 3D stiffness values components provided some insight of their involvement. For the case of 


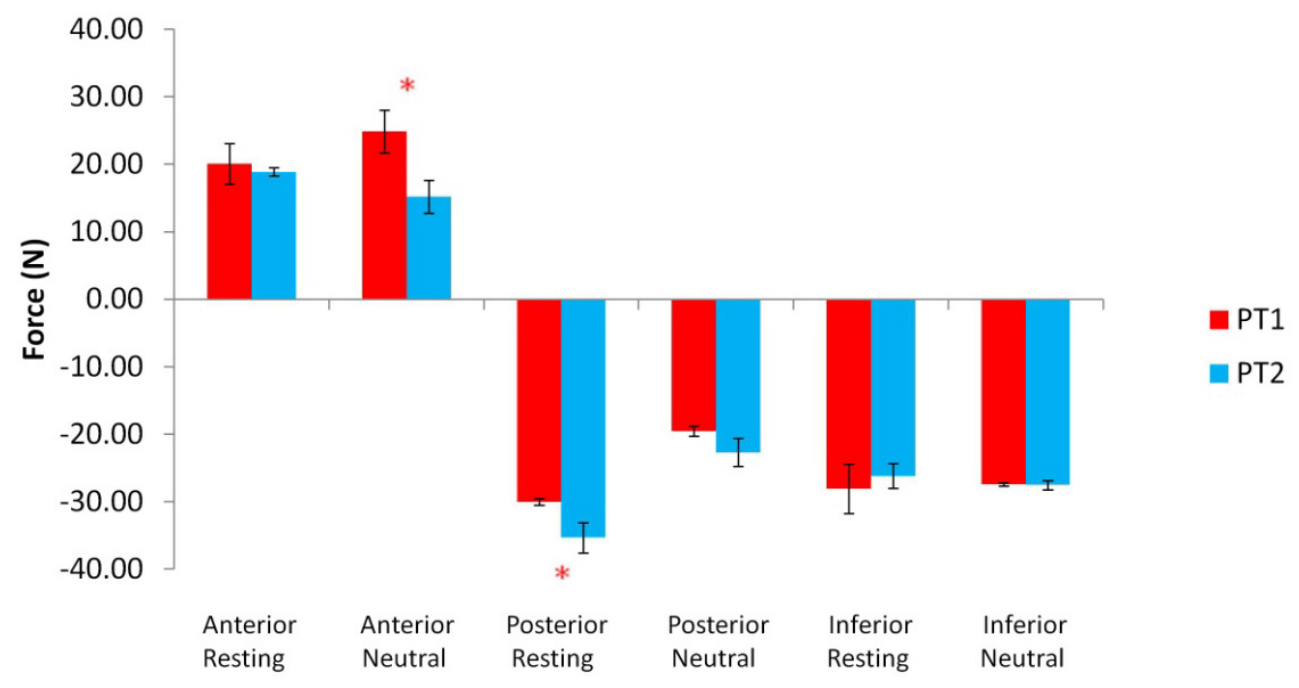

Figure 3-5. Comparison of mobilization axis forces between physical therapists. $*$ Signifies a statistically significant difference between therapists at $\mathrm{p}<0.05$. 
Table 3-3. 3D forces (N), positions ( $\mathrm{mm})$, and stiffness values ( $\mathrm{N} / \mathrm{mm})$.

\begin{tabular}{|c|c|c|c|c|c|c|c|}
\hline \multirow[b]{2}{*}{ Glide } & \multirow[b]{2}{*}{ Therapist } & \multicolumn{2}{|c|}{ 3D Forces } & \multicolumn{2}{|c|}{ 3D Position } & \multicolumn{2}{|c|}{ 3D Stiffness } \\
\hline & & Mean & SD & Mean & SD & Mean & SD \\
\hline \multirow[t]{2}{*}{ Anterior Rest } & PT1 & 24.4 & 2.1 & 11.7 & 3.4 & 2.2 & 0.5 \\
\hline & PT2 & 30.1 & 0.6 & 15.7 & 0.3 & 1.9 & 0.0 \\
\hline \multirow[t]{2}{*}{ Anterior Neutral } & PT1 & 32.6 & 3.9 & 21.3 & 3.2 & 1.5 & 0.2 \\
\hline & PT2 & 25.3 & 2.9 & 17.2 & 2.8 & 1.5 & 0.1 \\
\hline \multirow[t]{2}{*}{ Posterior Rest } & PT1 & 30.5 & 0.7 & 9.2 & 1.6 & 3.4 & 0.5 \\
\hline & PT2 & 43.4 & 0.7 & 6.6 & 1.4 & 6.7 & 1.5 \\
\hline \multirow[t]{2}{*}{ Posterior Neutral } & PT1 & 24.6 & 3.6 & 14.5 & 1.8 & 1.7 & 0.1 \\
\hline & PT2 & 23.0 & 1.9 & 19.5 & 3.8 & 1.2 & 0.2 \\
\hline \multirow[t]{2}{*}{ Inferior Rest } & PT1 & 35.8 & 4.4 & 26.5 & 2.8 & 1.4 & 0.2 \\
\hline & PT2 & 29.8 & 1.7 & 30.4 & 3.5 & 1.0 & 0.1 \\
\hline \multirow[t]{2}{*}{ Inferior Neutral } & PT1 & 27.4 & 0.2 & 21.1 & 3.4 & 1.3 & 0.2 \\
\hline & PT2 & 19.0 & 1.2 & 19.3 & 4.0 & 1.0 & 0.2 \\
\hline
\end{tabular}

N: Newton, PT1: Physical Therapist 1, PT2: Physical Therapist 2, SD: Standard Deviation.

Table 3-4. Component forces $(\mathrm{N})$ during posterior resting glide.

\begin{tabular}{lccrrrrr}
\hline & \multicolumn{3}{c}{ PT1 } & & \multicolumn{3}{c}{ PT2 } \\
\cline { 2 - 4 } \cline { 6 - 8 } Run & Fx & Fy & Fz & & Fx & Fy & Fz \\
\hline 1 & -2 & 3.4 & -29.7 & & -26.9 & -5.9 & -33.8 \\
2 & -3.7 & 4.3 & -29.7 & & -25.3 & -0.2 & -34.2 \\
3 & -4.1 & 5.5 & -30.6 & & -22 & 3.0 & -37.9 \\
Mean & -3.3 & 4.4 & -30.0 & & -24.7 & -1.0 & -35.3 \\
SD & 1.1 & 1.0 & 0.5 & & 2.5 & 4.5 & 2.2 \\
\hline
\end{tabular}

N: Newton, PT1: Physical Therapist 1, PT2: Physical Therapist 2, SD: Standard Deviation. 


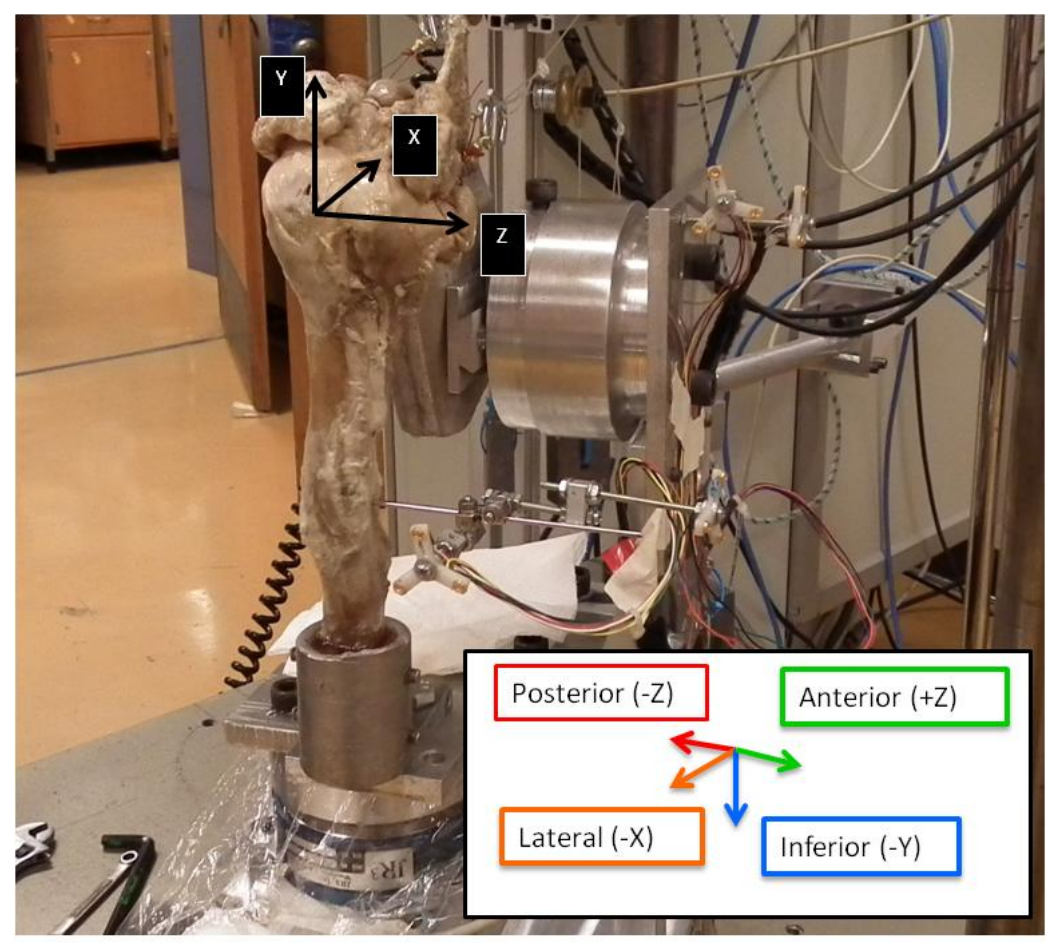

A)

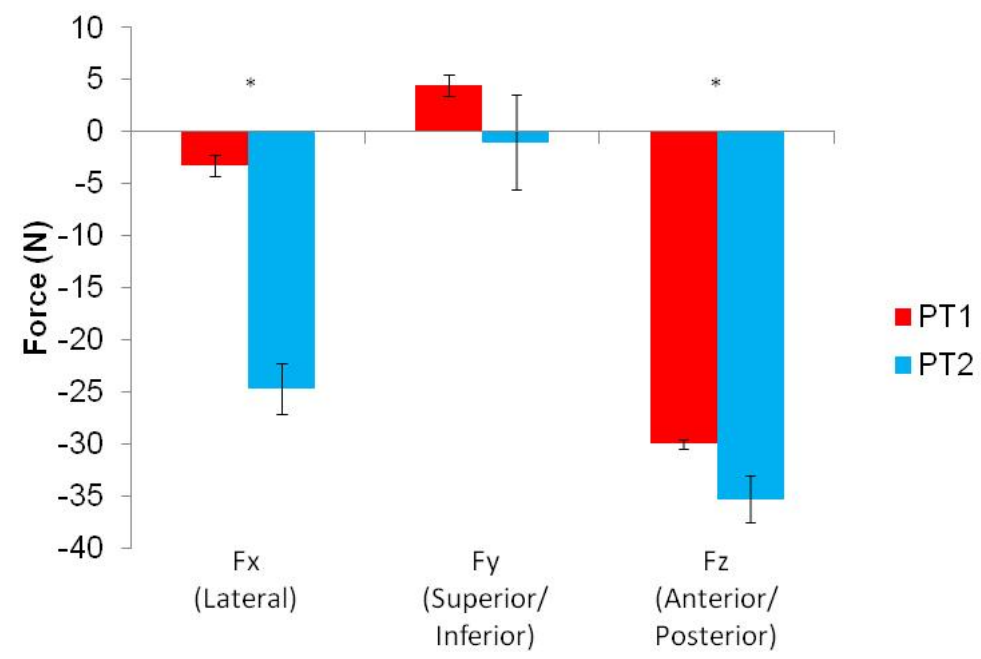

B)

Figure 3-6. Analysis of component forces during posterior glide.

(A) Picture of mounted specimen with coordinate system transferred to GH joint center.

(B) Forces along the non-mobilization (Fx and Fy) and mobilization (Fz) axes. PT2 applied a lateral force to the $\mathrm{GH}$ joint throughout the mobilization.

*Signifies a statistically significant difference between therapists for $\mathrm{p}<0.05$. 
the posterior resting mobilizations, an analysis of the non-mobilization forces (Figure 3-6) demonstrated that PT1 applied a lateral distractive force to the joint throughout the mobilization, while PT2 applied minimal forces along the lateral axis. The additional lateral distractive force applied by one of the PTs helped clear the humeral head from contacting the glenoid during the mobilization. There were only two other situations where the end forces applied by the two PTs were different and that occurred during posterior mobilization in the resting position and anterior mobilization in the neutral position. However, for both of these cases the differences in the mobilization axis forces were between $5 \mathrm{~N}$ and $10 \mathrm{~N}$ and may have limited clinical relevance.

The PTs were compared using 3D forces and displacements. Hsu et. al (2009) compared PTs on a single direction and found large force coefficients of variation (40\% to $77 \%$ ). Using 3D force comparisons our coefficients of variation ranged from $1.6 \%$ to $14.6 \%$ with an average of $7.6 \%$. This technique of data comparison emphasizes the total force and displacement that occurred at the joint. For example, a PT who applies distraction (-X) along with a posterior glide (-Z) and a small superior pre-positioning $(+Y)$ would have all of these forces included in the mobilization.

Moving the GH joint to a more close pack alignment, as in the resting position, is thought to tension the surrounding tissue and stiffen up the joint (Hertling and Kessler., 2006). This may help explain why the mobilization forces were significantly higher during the posterior glides compared with the neutral position.

As with any in vitro study, a number of limitations exist. The cadaveric model lacked active muscle loading and should not be generalized to therapeutic stretching techniques. Muscle tone of the $\mathrm{RC}$ muscle was simulated with a static load throughout the mobilization. However, during in vivo mobilizations of the GH joint, other muscles, including the RC muscles, can actively respond to manual therapy treatment. Patients with injured shoulders will sometimes guard their shoulder by using $\mathrm{RC}$ muscles to stabilize the joint. This study was unable to simulate patient guarding. Although the forces simulated in this study were much lower compared to literature, similar GH joint displacements have been reported by others (Hsu et al., 2009).

Although removing the tissue superficial to the rotator cuff muscles and musculature surrounding the humerus reduced the size and shape of the lower arm, the PTs did not detect any notable difference in the GH joint stiffness response. Future studies could include addition of sculptured foam pads to recreate the size, shape and feel of the lower arm.

Only one cadaveric specimen was used for all experimental mobilizations and the lab temperature was below body core temperature which may have affected tissue properties. Generalization to living patients should be cautioned. Likewise, direct comparison of the absolute end range of motion forces and displacements (and resultant $\mathrm{GH}$ joint stiffness values) with other equivalent in vitro data from the literature may be limited. Never-the-less, this method is quite feasible for comparing mobilization techniques between different Therapists. In this study the end range maximal forces were 
similar for 4 of the 6 mobilizations and the end range stiffness values demonstrating the consistent respond in techniques and tissue properties. Likewise the in vitro GH stiffness values reported here for the anterior and posterior glides were within one standard deviation of the in vivo findings reported by McQuade et al. for comparable mobilizations (McQuade et al., 2012). Figure 3-7 compares in vivo stiffness data with recorded anterior/posterior glide stiffness from this study. Each of these studies looked at different degrees of abduction for the glide.

Lastly the forces measured were applied by PTs and represent the inherent passive tissue properties are on the cadaveric specimen for a subset of mobilization techniques. In clinical practice, the mobilization forces used and the mechanical tissue responses of the tissue might differ from those used in this study, depending on such factors as pain, inflammation, muscle spasm, co-contraction, and pathology of the GH joint.

\section{Conclusion}

A novel simulation model was developed that measured the biomechanical properties of the GH joint. The model was used to study three different Physical Therapy mobilization techniques as well as the effects of changing the joint's alignment position.Future work will include investigation the effects of ligamentous injuries and surgically altered conditions on glenohumeral biomechanics. 


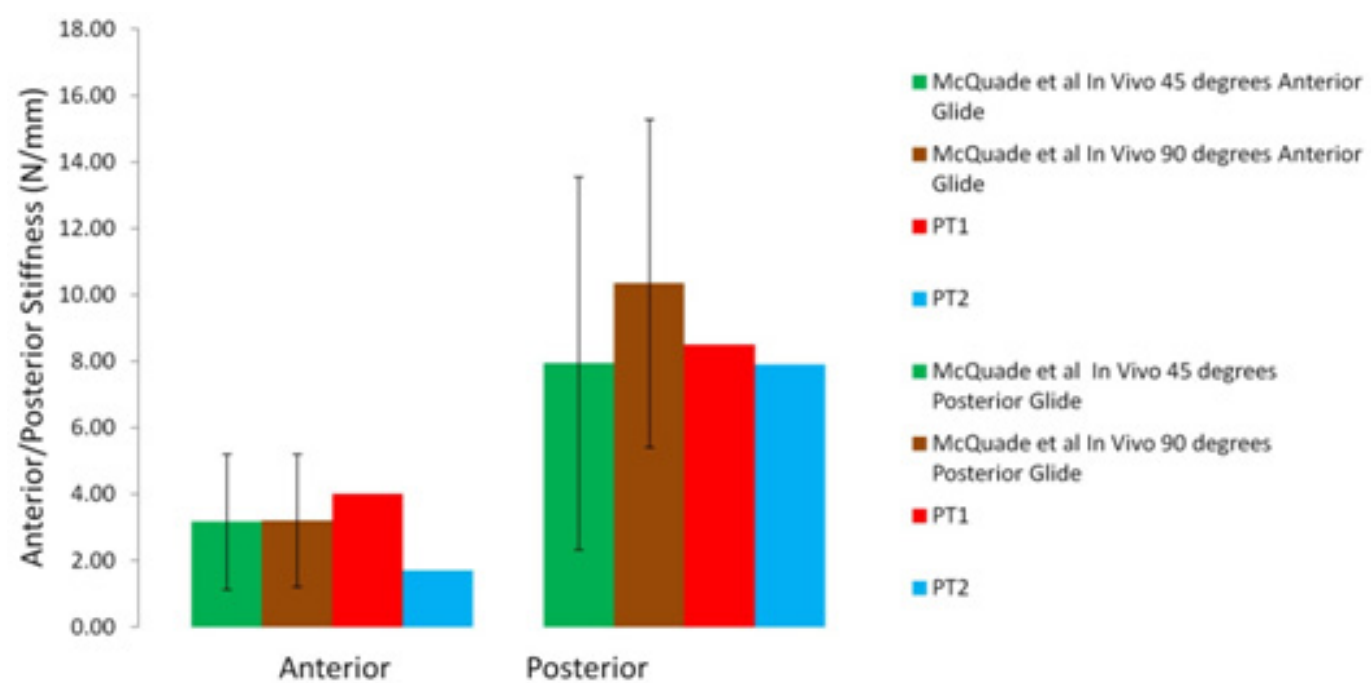

Figure 3-7. Comparison between in vivo and in vitro glides in the anterior and posterior directions.

Source: McQuade, K., Price, R., Liu, N., and Ciol, M. "Objective Assessment of Joint Stiffness: A Clinically Oriented Hardware and Software Device with an Application to the Shoulder Joint." Physiotherapy (2012). 


\section{CHAPTER 4. BIOROBOTIC SIMULATION OF GLENOHUMERAL JOINT MOBILIZATION TECHNIQUES}

Chapter 4 contains the summary of the manual therapy simulation experiment. The purpose of this study was to replicate forces and displacements applied by therapists during a gliding treatment. The work described in this chapter will be submitted as a manuscript to the Journal of Biomechanics.

\section{Introduction}

Outpatient treatment for shoulder dysfunction remains one of the most common referrals for Physical Therapists (PTs) (Van der Windt and Butler, 2003). Hypomobility of the glenohumeral $(\mathrm{GH})$ joint can occur from shoulder impingement syndrome or adhesive capsulitis which can lead to shoulder pain and immobility (Kelley et al., 2009, Yiasemides et al., 2011, Brudvig et al., 2011). Physical therapists employ joint mobilization (MOB) techniques to treat capsular hypomobility with the goal of reducing pain and increasing range of motion (Kelley and Leggin, 2009). The joint is positioned appropriately (e.g., neutral, resting, or end range) and an external force is applied to a patient's joint along a specific axis or direction to produce some deformation of the targeted connective tissue structures and displace the bones at either end of the joint (Hsu et al., 2002, Hertling and Kessler, 2006). The rate of increase in force resistance is a function of the structural stiffness properties of the joint (Hsu et al., 2000). If the mobilization force is excessive, micro-damage can occur to the joint capsule. On the other hand, too low of a mobilization force may not provide adequate stretch of the targeted tissue and the desired improvement in the range of motion to the joint may not be achieved. The MOBs are usually done in the anatomical planes. MOBs are typically done in an 'open pack' joint position versus a more 'closed pack' position. Although MOBs are a common therapy for treating joint pain and establishing ROM, the biomechanical effects associated with them have not been well studied (Farrell, 1992, Bryce et al.,2010, Ho et al., 2009, Hsu et al., 2000, Hsu et al., 2002). Further, the possibility of other joint treatment positions having greater clinical impact still exist and have not been ruled out. New, more effective joint orientations could be defined. To our knowledge, a truly quantified study exploring an array of GH joint positions had not been performed (Hertling and Kessler, 2006, Clarkson, 2000, Palmer and Epler, 1990, McQuade et al., 2012, Hsu and Headman,2002, Yiasemides et al., 2011, Voycheck et al., 2011, Browe et al., 2011, Karduna et al., 2005, Wueler and Wirth, 1995).

More recently biorobotic testing platforms have been introduced to the field of joint biomechanics. They are highly controllable devices that enable improved simulation of the physiological loading and motion conditions that occur in vivo. These systems are ideal for investigating the dynamic effects of changing the direction of the mobilization force and/or the joint position (Hsu and Headman, 2006). A variety of biorobotic systems have been used to simulate dynamic forces in up to four degrees of freedom (DOF) (Browe et al., 2011, Voycheck et al., 2011, Hsu and Headman, 2002, Karduna et 
al., 2005, Bryce et al., 2010). Even with this vast number unique robotic designs, only a select few of these setups have ventured to explore the biomechanical effects of joint gliding (Hsu physical therapy, Hsu arch phys med rehabil). These limited studies only simulated anterior/posterior glides, which only account for a fraction of the MOBs used in clinics (Hertling and Kessler, 2006).

The long term goal of this research is to explore joint positions and/or direction of the mobilization forces that result in optimal tissue stretching for a given MOB glide and to ensure existing and new MOB techniques are consistent, safe, and effective by using an advanced biorobotic testing platform. The objective of this study was to establish a testing method for robotic simulation of existing mobilization techniques used to treat hypomobility of the GH joint. GH mobilization therapies investigated in this study included anterior, posterior, and inferior glides with varying internal and external rotation during joint flexion $(\mathrm{F})$ and extension $(\mathrm{E})$. Internal/external rotations were investigated as a means of validating joint open/closed pack positions.

\section{Methods}

\section{Tissue Preparation}

One right human cadaveric shoulder (Male, Age: 58 years) was procured and prepared for testing. All superficial soft tissue was removed until the RC muscles and tendons were exposed. Monofilament nylon was sutured to the RC tendons of the subscapularis, supraspinatus, and teres minor/infraspinatus using a modified locking suture technique. (Krackow et al., 2011) The teres minor and infraspinatus share a similar magnitude; both were combined into a single muscle line for model simplicity. The humerus was cut approximately $230 \mathrm{~mm}$ from the joint center as shown in

Figure 4-1, and the distal end was potted with $10 \mathrm{~N}$ of a low melting point bismuth alloy making the final weight of the humerus and potting material $15 \mathrm{~N}$. The inferior angle of the scapula was then potted in more bismuth alloy with the scapula in a neutral upright orientation. After being prepped, the specimen was placed in an airtight container and stored at $-10^{\circ} \mathrm{F}$. Prior to testing the frozen specimen was thawed 24 hours at $40^{\circ} \mathrm{F}$.

\section{BioRobotic Testing Platform and Specimen Mounting Protocol}

An existing programmable, multi-axis Robot Testing Platform (RTP) (Kelly and DiAngelo, 2006) was modified to accommodate the anatomy of the shoulder and rotator cuff muscle group. The RTP consisted of a 4 degree of freedom (DOF) fully controllable robotic system arranged in a Cartesian coordinate system (Figure 4-2). Rotary motors were upgraded from the previous frame's rotational $\mathrm{Y}$ and $\mathrm{Z}$ axes for a higher encoder resolution $\left(0.0002^{\circ}\right)$ and load capacity $(260 \mathrm{Nm})$. The rotary axes were arranged as a gimbal assembly. A six axis load cell attached to the gimbal assembly measured the three dimensional (3D) Cartesian forces and moments applied to the specimen. The potted 


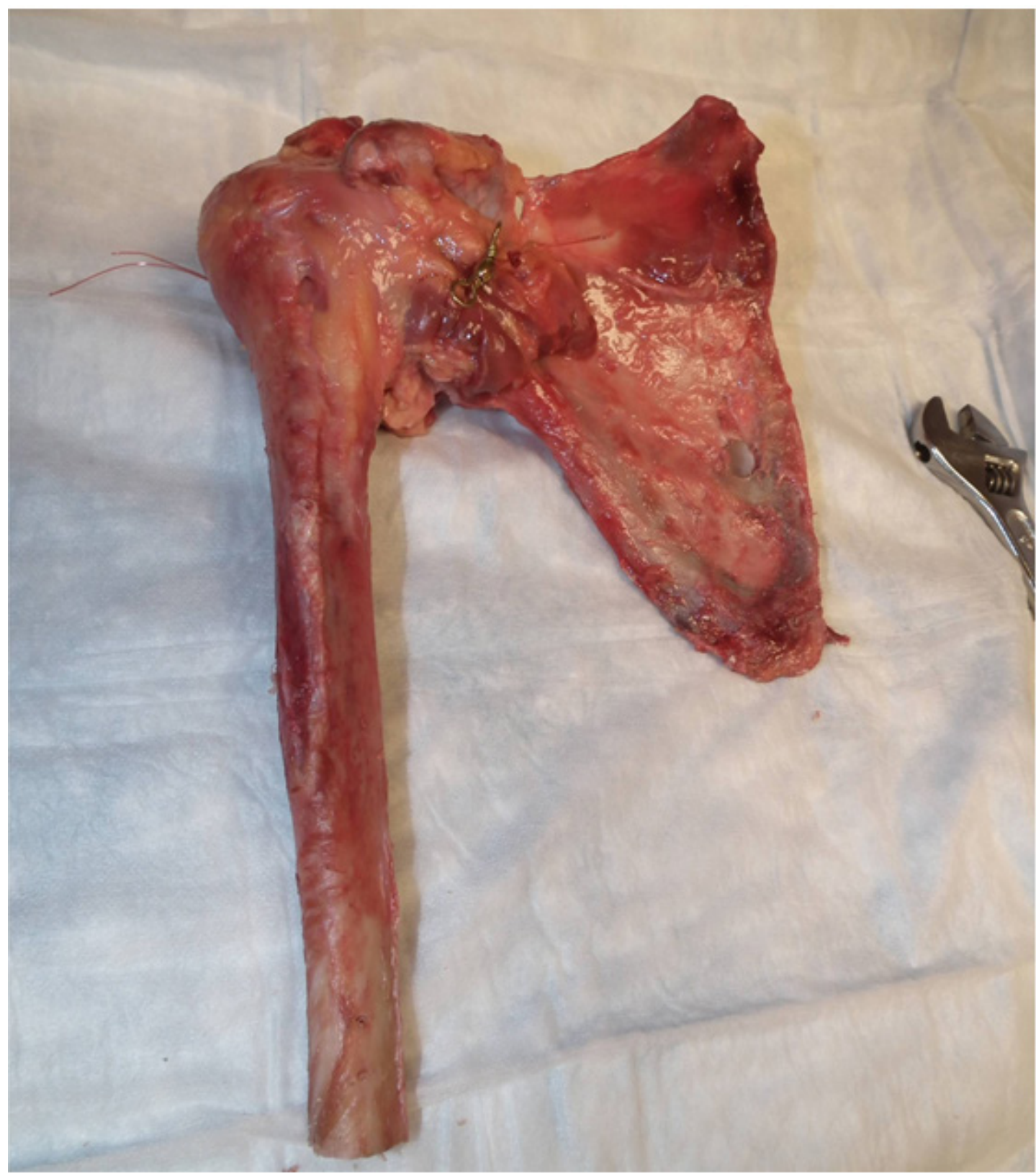

Figure 4-1. Photograph of the prepared GH joint specimen.

Monofilament nylon was sutured into the humerus soft tissue at the origin of the RC muscles. 


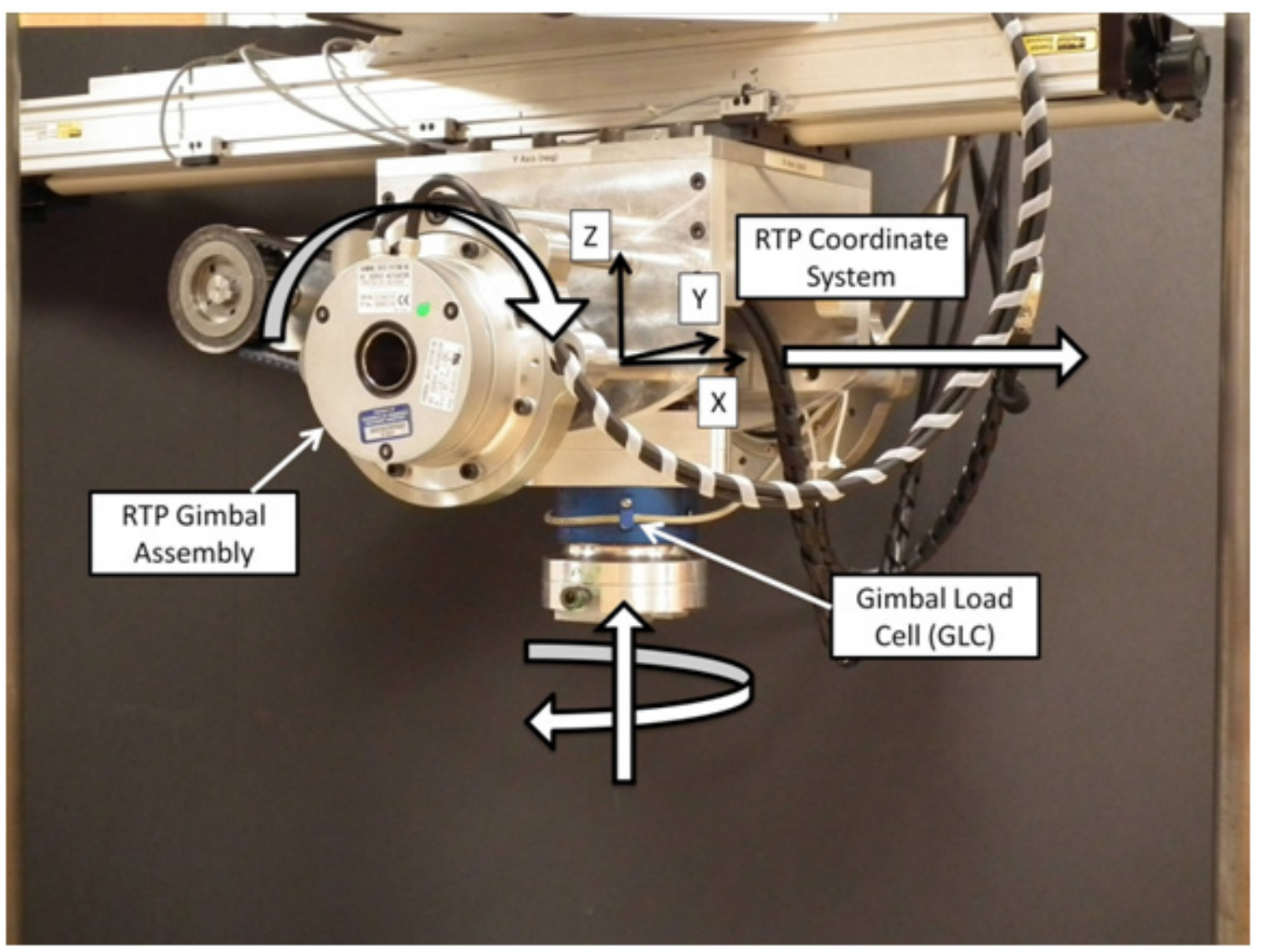

Figure 4-2. Arrangement of four controlled axes of the RTP. 
specimen was then rigidly mounted to the RTP in an inverted upright neutral position with the anatomical planes aligned with the robotic world coordinate system

(Figure 4-3): $x$-axis motor controlled specimen anterior/posterior motion and forces; $z$ axis motor controlled superior/inferior motion and forces; pitch motor (rotation about the $\mathrm{y}$-axis) controlled $\mathrm{F} / \mathrm{E}$; roll motor (rotation about the $\mathrm{z}$-axis) controlled humeral internal/external rotation. Because the humerus was rigidly connected to the RTP, coordinated motion of motion and forces of the RTP axes were transformed to the GH joint center. The gimbal assembly and GLC loads were transformed to and controlled at the $\mathrm{GH}$ joint center using rigid body mechanics.

\section{GH Joint Mechanics}

A schematic of the test setup with the specimen mounted in an inverted neutral position is shown in Figure 4-4. An $x-y$ table was installed at the base of the system to introduce a fifth passive degree of freedom. This feature was used to engage the RC muscles through unconstrained lateral motion of the scapula relative to the humerus. A displacement transducer (Honeywell MLT 38000204) mounted to the base plate was attached to the $y$-axis of the $x-y$ table to capture medial-lateral motion. The table's $x$-axis remained locked throughout testing. A cable-pulley assembly attached to the scapula fixtures was used to load the $\mathrm{GH}$ joint. Weights suspended from the cables transferred load through the RC muscles and pull the scapula into the GH joint. The suspended weights were set to $5 \mathrm{~N}$ each resulting in a $\mathrm{GH}$ joint load of approximately $13 \mathrm{~N}$ including frictional losses.

\section{Simulation Protocol}

Robotically controlled forces simulating humeral loading were transformed to the $\mathrm{GH}$ joint center and balanced about the specimen sagittal plane within a tolerance of $1.0 \mathrm{~N}$. This did not affect the lateral static $5 \mathrm{~N} \mathrm{RC}$ muscle tone loads. The simulation protocol rotated the $\mathrm{GH}$ joint through $10.0^{\circ}$ of flexion and extension in half-degree increments about the glenohumeral joint center. Motion was coordinated synchronously about the GH joint to simulate physiologic motion. At each rotational increment the humerus was displaced in the sagittal plane (i.e., side to side and up and down) to minimized the forces about the GH joint center within a $2.0 \mathrm{~N}$ tolerance, defining a neutral balance point (BP). If at any point in testing the sagittal plane forces could not reach the $\pm 2.0 \mathrm{~N}$ tolerance limit, the balancing routine was terminated. After the balancing forces were reached at each incremental half-degree step, the GH joint position and forces were recorded (as the BP) and the specimen underwent translational mobilization at $1.3 \mathrm{~mm} / \mathrm{s}$ in the posterior, inferior, and anterior directions. Mobilization was terminated after $10.0 \mathrm{~mm}$ or $20.0 \mathrm{~N}$ along the active axis for anterior and poster gliding, and $10.0 \mathrm{~mm}$ or $15.0 \mathrm{~N}$ for inferior gliding. Forces and positions were recorded again after mobilization limits were reached, defining the displaced point (DP). Figure 4-5 shows the BP and DP positions for a posterior mobilization. After the anterior, 


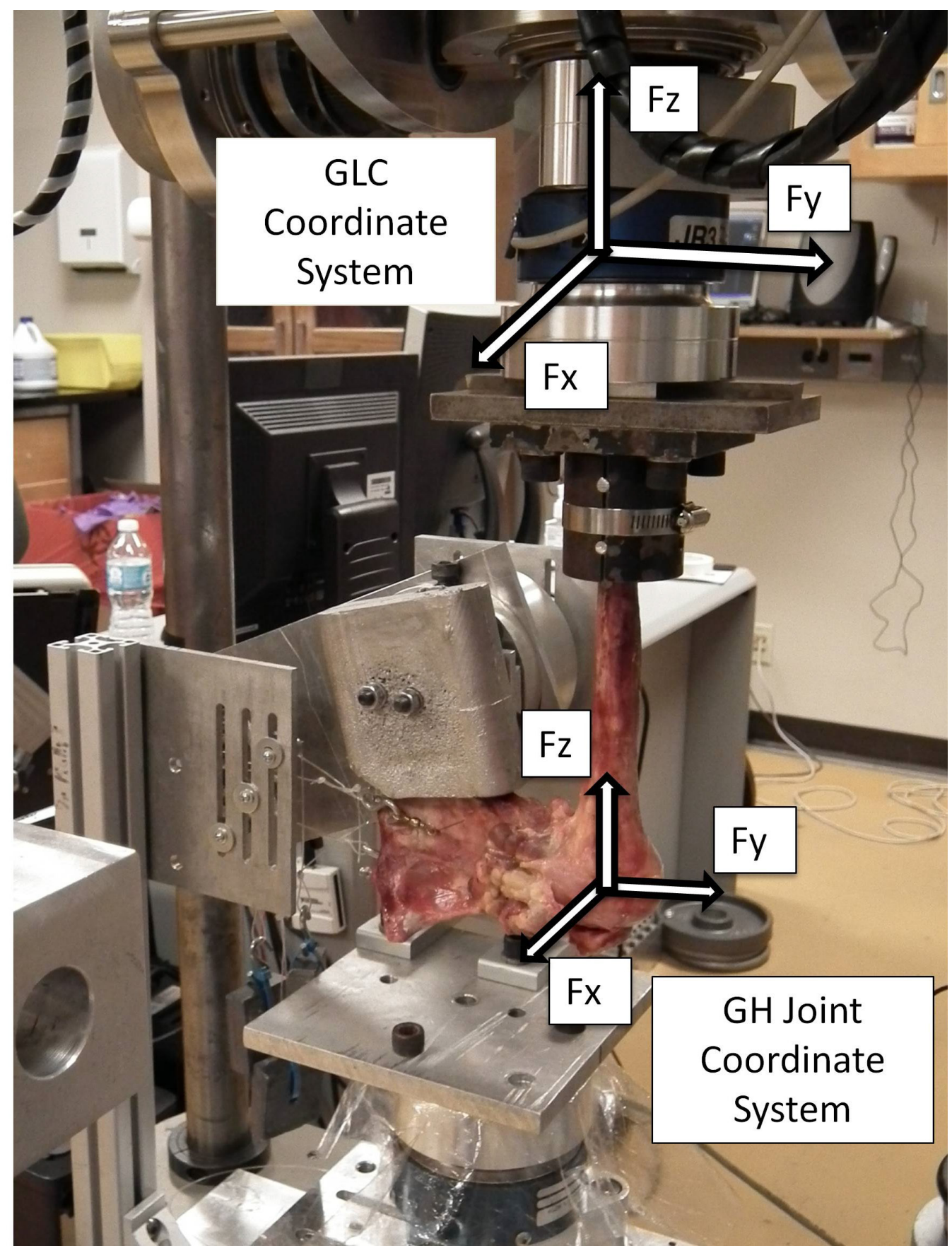

Figure 4-3. Test setup with the specimen mounted and rotator cuff loads engaged. Motion and forces of the RTP axes were transformed to the GH joint center. 


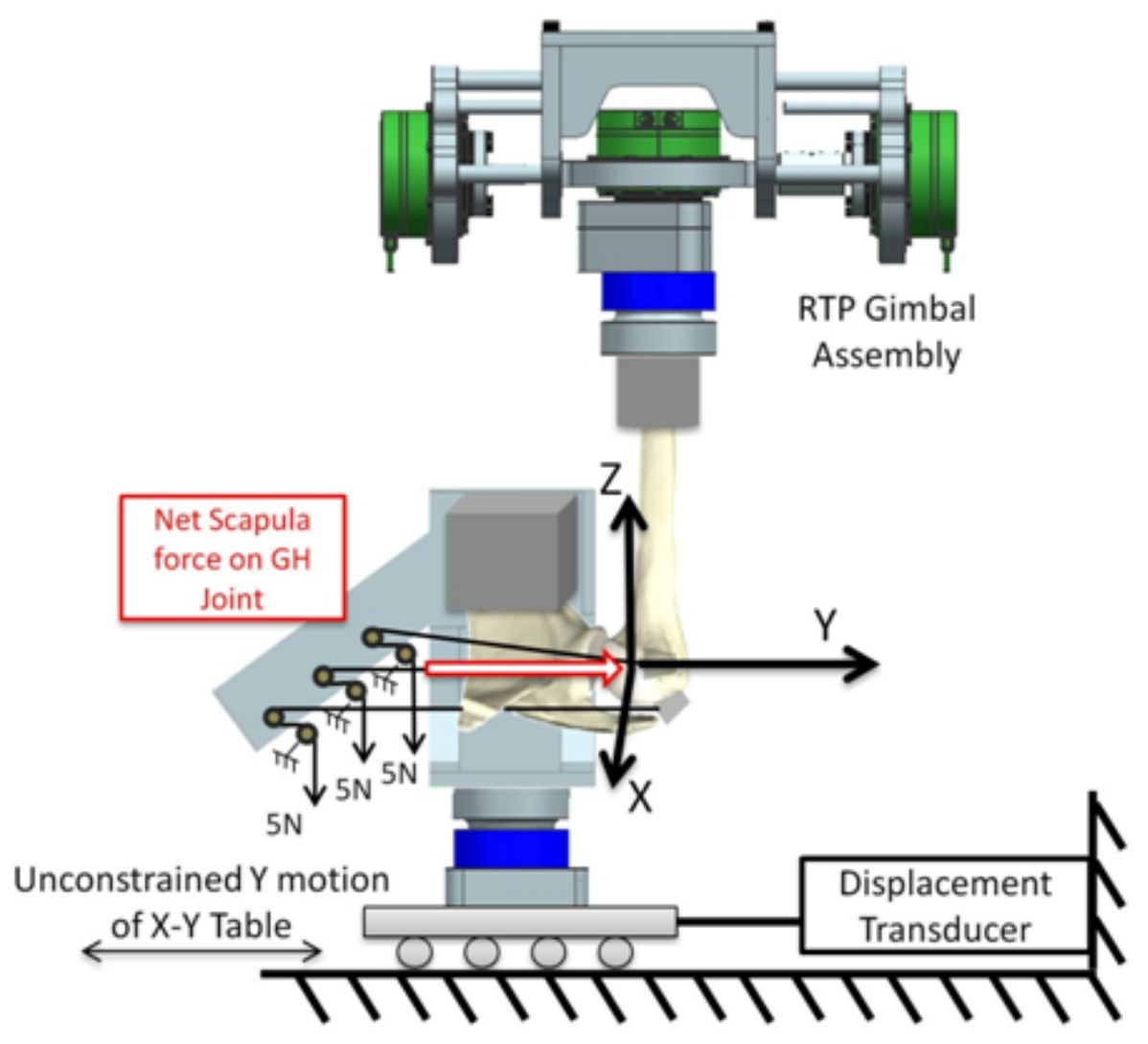

Figure 4-4. Schematic of the fixture system used to simulate RC muscles.

Motion in the sagittal plane was unconstrained allowing the RC loads to remain active throughout the mobilization. 


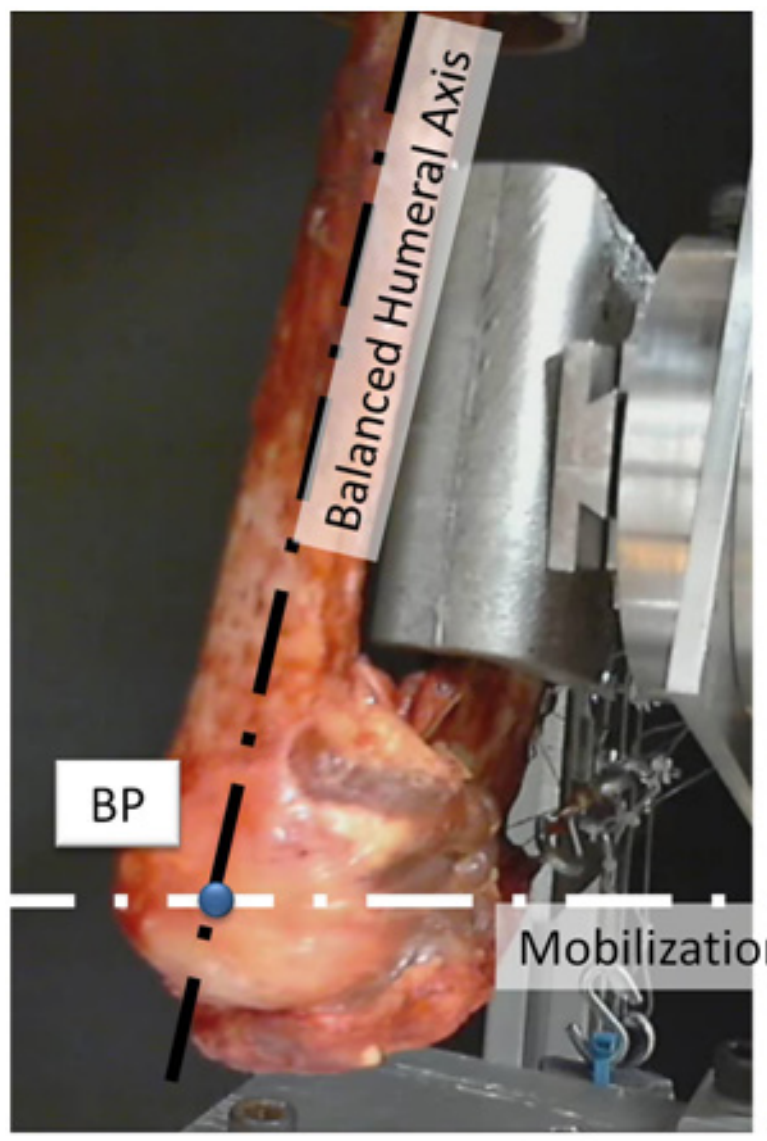

Balanced Point (BP)

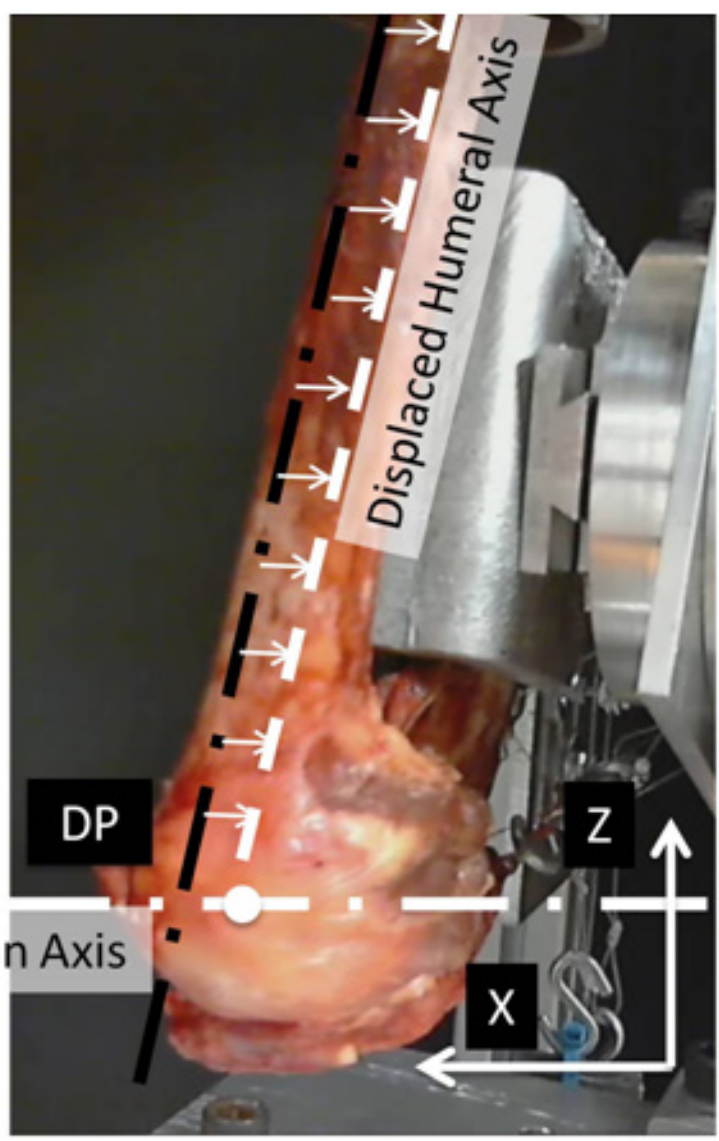

Displacement Point (DP)

Figure 4-5. Specimen in premobilized (BP) and postmobilized (DP) position. Specimen is undergoing a posterior glide. 
posterior, and lateral mobilizations tests were completed with the specimen in the neutral position, the 3 mobilizations tests were repeated with the humerus rotated internally $30^{\circ}$ and then externally $30^{\circ}$.

\section{Data Management}

The 3D forces and positions of the GH joint were recorded at each BP and DP in three dimensional Cartesian coordinates. Stiffness values were then calculated by the difference between the forces and positions recorded in the BP and DP as shown in Equation 4-1, Equation 4-2, and Equation 4-3. Joint stiffness was analyzed to compare the effects of IR/ER and F/E on the 3D stiffness of the joint. Joint position was then analyzed to compare the effects of IR/ER and F/E on the $3 \mathrm{D}$ displacement of the joint. Effect comparisons for 3D stiffness and 3D displacements were analyzed for the Anterior, Posterior, and Inferior glides.

The mean 3D stiffness values were calculated over the range of $\mathrm{F} / \mathrm{E}$ motion for each of the glide directions. A One Way Analysis of Variance with an SNK post-hoc test with $p=0.05$ was used to determine the significant differences in the $3 \mathrm{D}$ stiffness values during F/E for the mean of each glide groups. When normality tests failed, Dunn's method was used.

$$
\begin{gathered}
3 D \text { Force }=D P_{\text {Force }}-B P_{\text {Force }} \\
3 D \text { Position }=D P_{\text {Position }}-B P_{\text {Position }} \\
3 D \text { Stiffness }=\frac{3 D \text { Force }}{3 D \text { Position }} *
\end{gathered}
$$

*When the forces at the BP are near zero, the 3D Stiffness values represent the actual stiffness of the joint.

A One Way Analysis of Variance with an SNK post-hoc test with $p=0.05$ was used to determine the significant differences in the $3 \mathrm{D}$ stiffness values during $\mathrm{F} / \mathrm{E}$ for the three glide groups.

\section{Results}

The mean 3D stiffness values were calculated over the range of $\mathrm{F} / \mathrm{E}$ motion for each of the glide directions and GH joint positions and are listed in Table 4-1. Anterior, Posterior, and Inferior charts comparing the effects of F/E and IR/ER of 3D stiffness were plotted in Figure 4-6, Figure 4-7, and Figure 4-8 respectively. Start position (neutral joint position) 3D stiffness values (Table 4-1) were tabled to compare the differences in stiffness between glide's joint positions (F/E with IR/ER) before joint flexion or extension. 
Table 4-1. Neutral position 3D stiffness values $(n=2)$.

\begin{tabular}{lcc}
\hline Glide & Rotation & Mean \pm SD (N/mm) \\
\hline Anterior & Neutral & $3.61 \pm 0.05$ \\
& Internal & $3.06 \pm 0.26$ \\
& External & $8.25 \pm 0.28$ \\
Posterior & Neutral & $1.60 \pm 0.07$ \\
& Internal & $4.40 \pm 0.06$ \\
& External & $8.03 \pm 0.48$ \\
\hline Inferior & Neutral & $3.02 \pm 0.97$ \\
& Internal & $5.39 \pm 0.21$ \\
& External & $12.74 \pm 4.32^{*}$ \\
\hline
\end{tabular}

N: Newton, SD: Standard Deviation. 


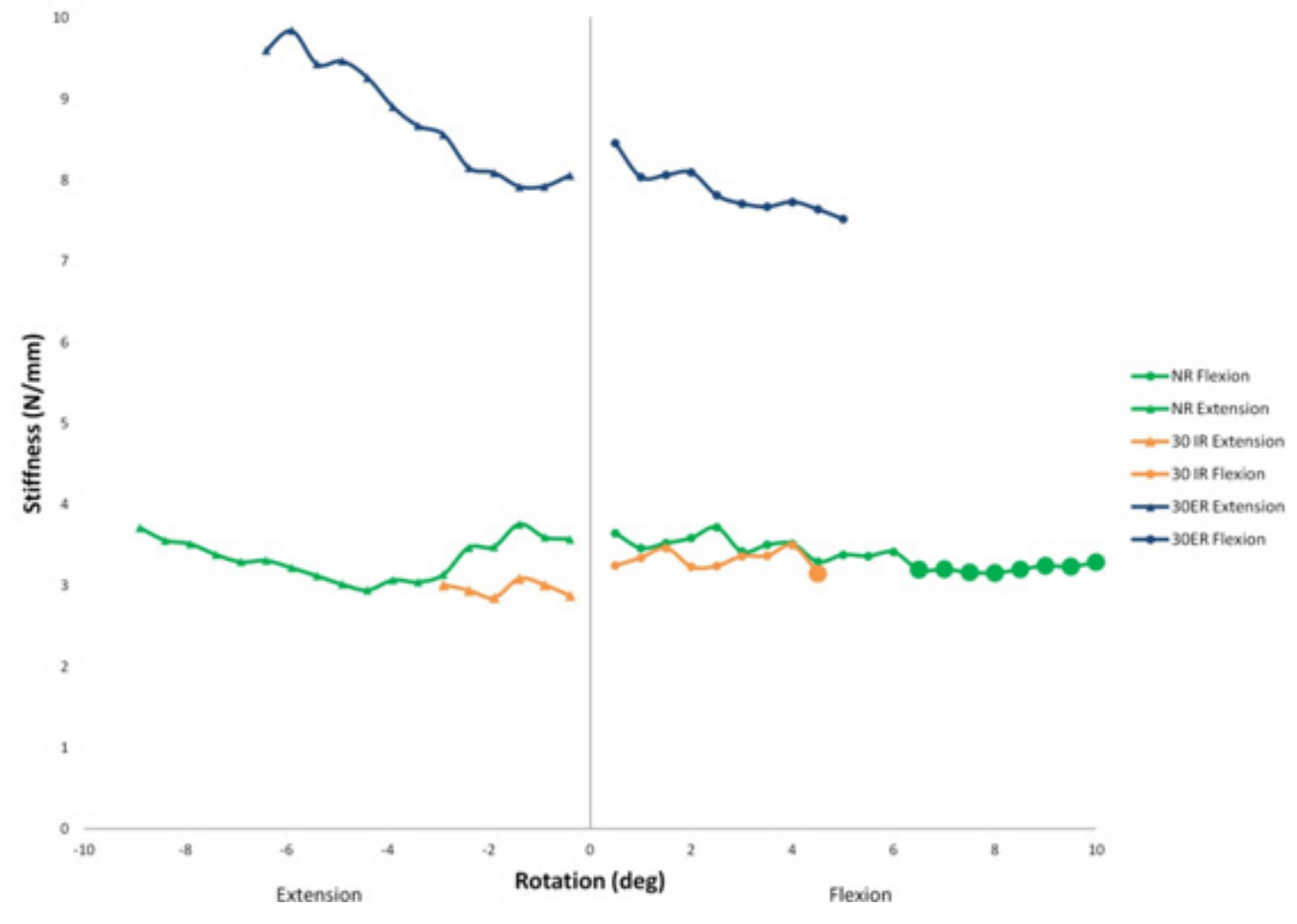

Figure 4-6. Anterior glide 3D stiffness graph.

Enlarged points represent locations where distance was the limiting factor. 


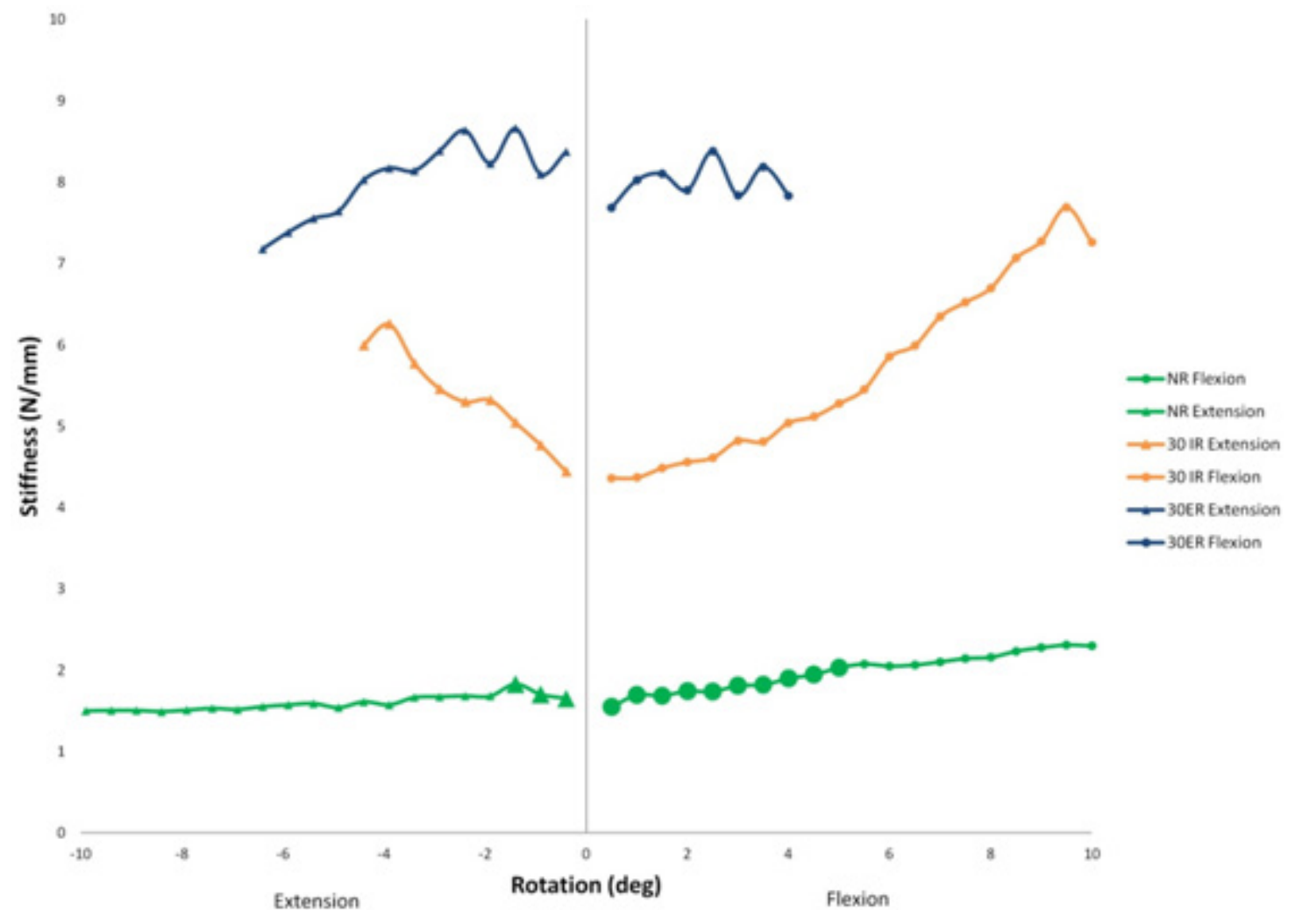

Figure 4-7. Posterior glide 3D stiffness graph.

Enlarged points represent locations where distance was the limiting factor. 


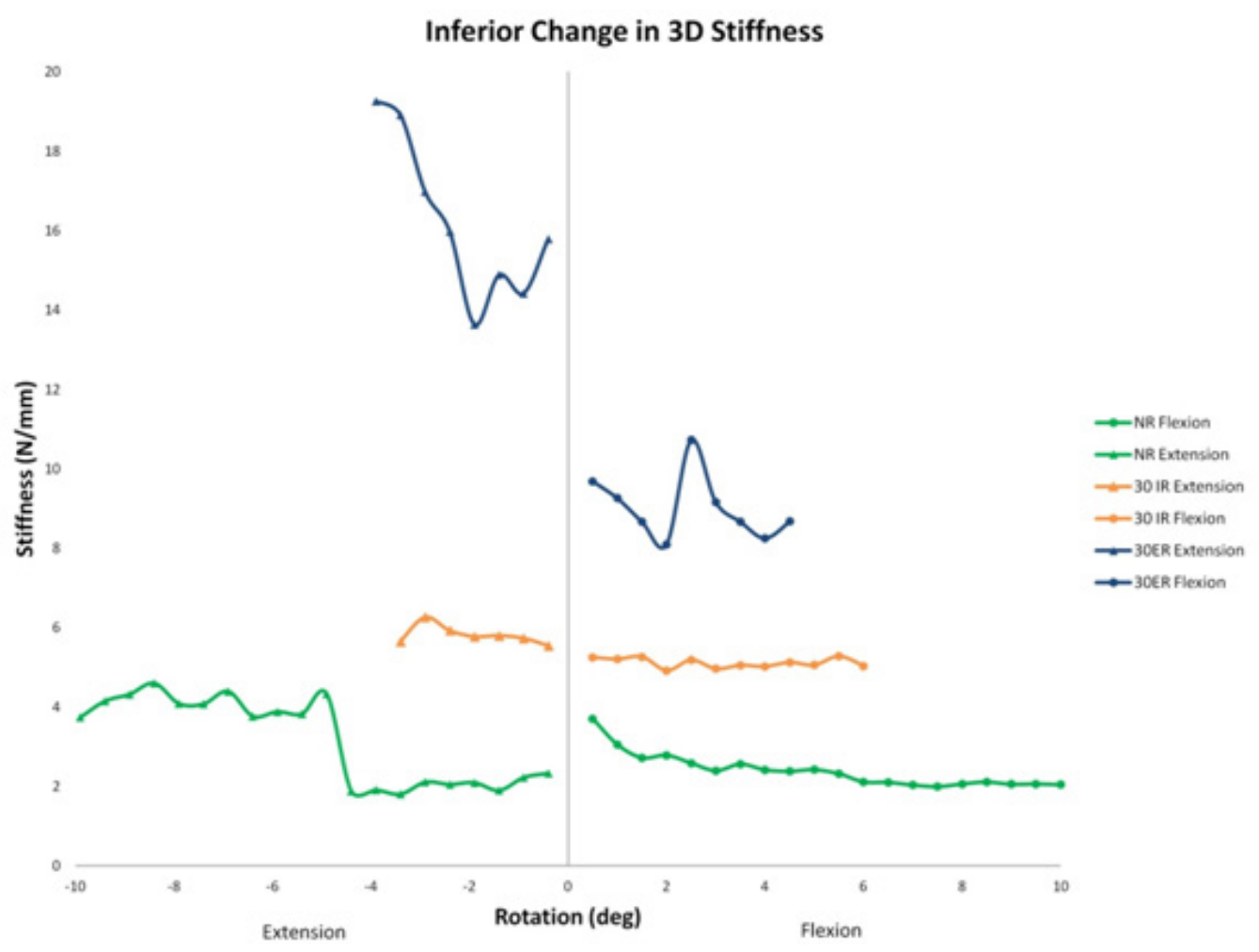

Figure 4-8. Inferior glide 3D stiffness graph.

Enlarged points represent locations where distance was the limiting factor. 
Statistically significant differences in the mean stiffness values for each glide's flexion/extension path are shown in Table 4-2. A complete set of absolute data from this experiment can be found in Appendix C. Pre-mobilization changes in GH joint axis of rotation following $30^{\circ}$ of IR/ER from the neutral position were compared as shown in Figure 4-9 $(n=6)$. Rotating the joint externally caused a lateral shift in the joint's position along with a slightly inferior displacement. Internally rotating caused posterior joint motion along with a large magnitude of inferior motion. Anterior, Posterior, and Inferior displacement charts comparing the effects of $F / E$ and IR/ER of $3 D$ postmobilization changes in GH joint axis of rotation are shown in Figure 4-10, Figure 4-11, and Figure 4-12 respectively.

\section{Anterior Glide}

In the Anterior Glide (Figure 4-7), 30 of ER increases the stiffness to an anterior glide during both flexion and extension. External rotation slides the head of the humerus to a more anterior position and therefore the amount of "slack" is limited as shown in Figure 4-10. Extending the joint appeared to reduce the amount of displacement of the glide, while flexing increase the glide displacement. This suggests flexing the joint reduces the joint stiffness for an anterior glide. Thirty degrees of internal rotation which slides the humeral head posteriorly is no different from neutral in terms of an anterior glide during flexion and extension, although the amount of motion along the axis of mobilization was slightly lower in IR when compared to NR. If the head of the humerus is in the center or positioned posteriorly there is "slack" present and stiffness does not increase during and anterior glide. Keep in mind that the arm is at $0^{\circ}$ of abduction when the anterior glide is produced by the robot.

\section{Posterior Glide}

For the Posterior Glide (Figure 4-8), $30^{\circ}$ of ER caused an increase in stiffness during a posterior glide during flexion or extension. The stiffness is less than during anterior glide possibly due to the more anterior position the humeral head during anterior glide. The increased stiffness during external rotation suggests that even at $30^{\circ}$ of ER the capsule is winding up and moving the humeral head tighter to the glenoid. The increase in stiffness for posterior glide during internal rotation may to due to the posterior glide that occurs during IR. This would mean that there is less "slack" present during the posterior glide. The stiffness increases during flexion and extension at $30^{\circ}$ of IR. This may indicate that the relatively small degree of flexion and extension either tightens the capsule or moves the head of the humerus into a more posterior position. Figure 4-11 demonstrates how mobilizing the joint in ER in comparison to NR caused a much lower change in position to reach the prescribed $20 \mathrm{~N}$ force. Flexion reduces glide displacement in the NR and IR joint positions, although neutral position during flexion and extension did not increase the stiffness. No lateral distraction force was applied and stiffness did not increase as was expected. 
Table 4-2. Mean 3D stiffness values for individual glide paths.

\begin{tabular}{|c|c|c|c|}
\hline Glide & Path & GH Orientation & $\begin{array}{c}\text { 3D Stiffness }(\mathrm{N} / \mathrm{mm}) \\
\text { (Mean } \pm \text { Standard Deviation })\end{array}$ \\
\hline \multirow{6}{*}{ Anterior } & Flexion & Neutral & $3.37 \pm 0.17$ \\
\hline & & Internal & $3.32 \pm 0.11+$ \\
\hline & & External & $7.87 \pm 0.28^{*}$ \\
\hline & Extension & Neutral & $3.34 \pm 0.25$ \\
\hline & & Internal & $2.96 \pm 0.09+$ \\
\hline & & External & $8.76 \pm 0.70 *+$ \\
\hline \multirow[t]{6}{*}{ Posterior } & Flexion & Neutral & $1.98 \pm 0.23 *$ \\
\hline & & Internal & $5.68 \pm 1.10^{*}$ \\
\hline & & External & $7.99 \pm 0.23$ \\
\hline & Extension & Neutral & $1.59 \pm 0.09$ \\
\hline & & Internal & $5.37 \pm 0.58^{*}$ \\
\hline & & External & $8.04 \pm 0.47 *$ \\
\hline \multirow[t]{6}{*}{ Inferior } & Flexion & Neutral & $2.40 \pm 0.43$ \\
\hline & & Internal & $5.11 \pm 0.12 *+$ \\
\hline & & External & $9.02 \pm 0.81 *+$ \\
\hline & Extension & Neutral & $3.17 \pm 1.08^{*}$ \\
\hline & & Internal & $5.81 \pm 0.23^{*}$ \\
\hline & & External & $16.25 \pm 1.90 *$ \\
\hline
\end{tabular}

*Denotes statistically significant $(\mathrm{p}<0.05)$ differences relative to neutral orientation. + Denotes statistically significant $(p<0.05)$ differences between internal and external orientation. 


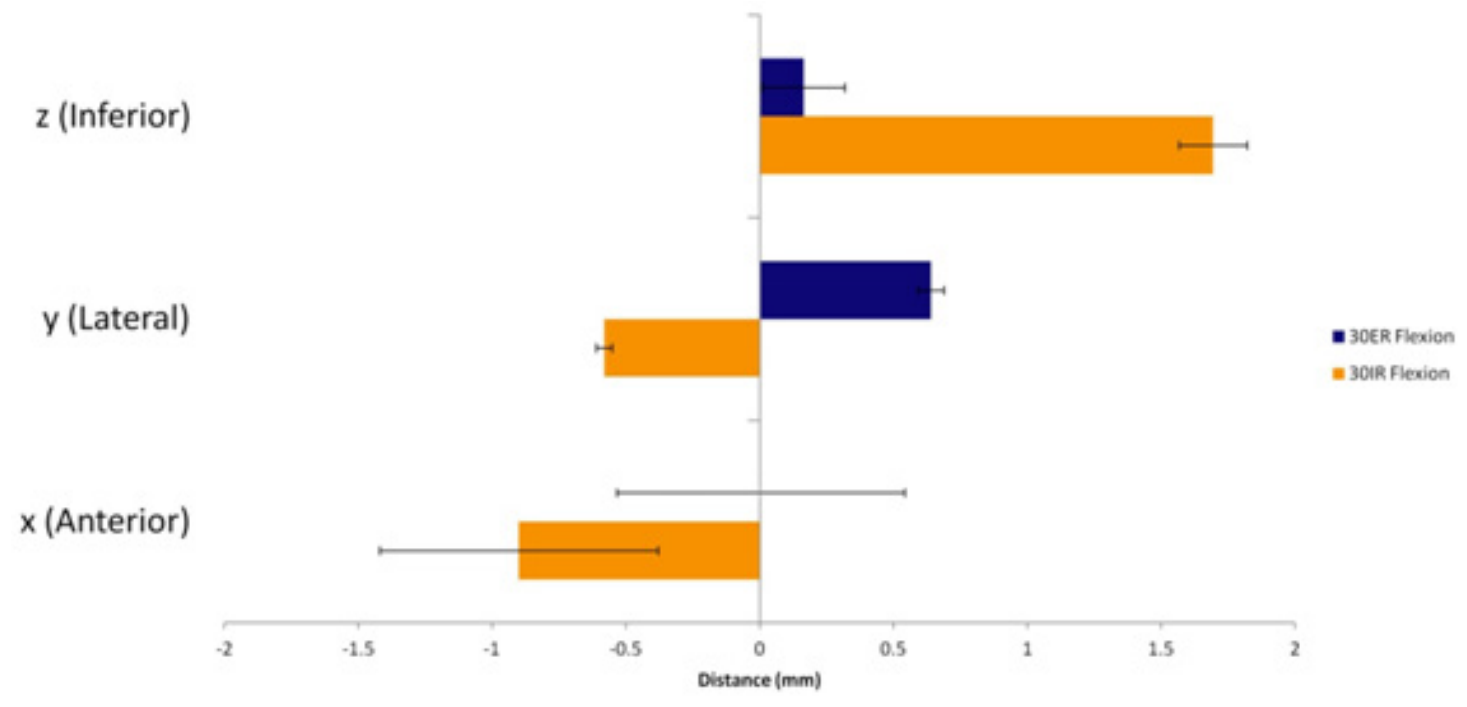

Figure 4-9. Effects of IR/ER on GH joint neutral position before mobilization. 

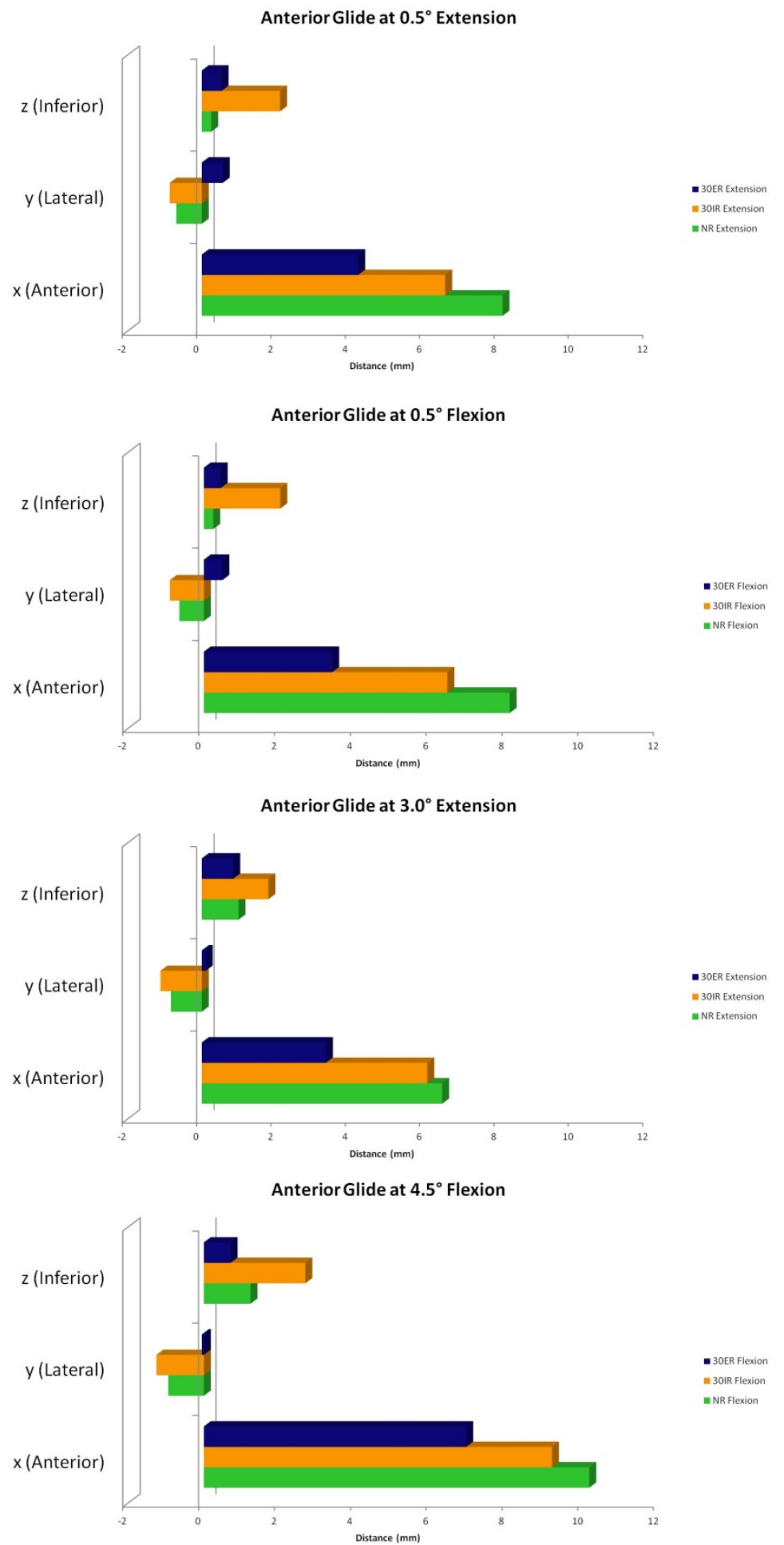

Figure 4-10. Effects of IR/ER and F/E on GH joint position before and after an anterior glide mobilization. 

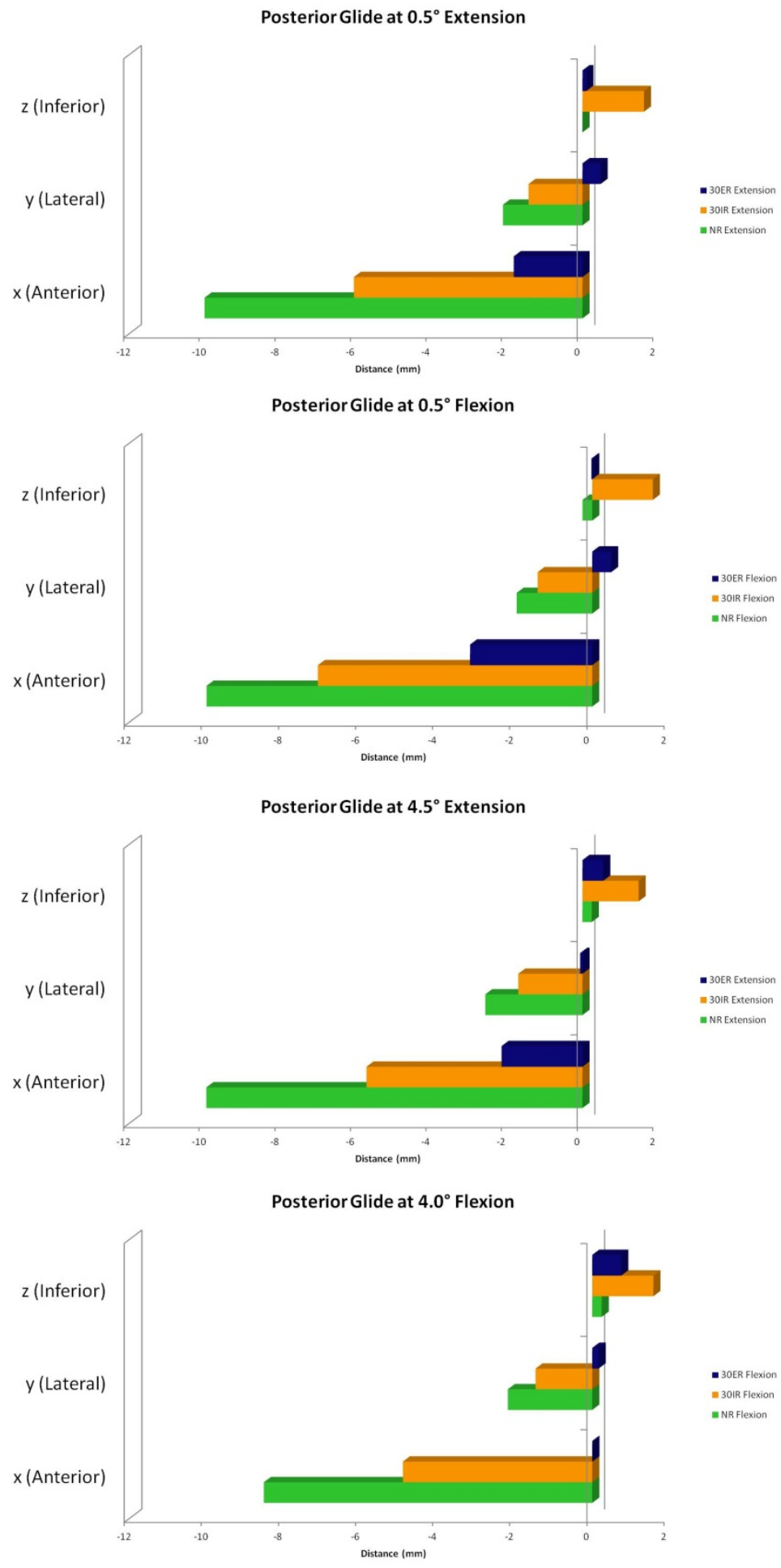

Figure 4-11. Effects of IR/ER and F/E on GH joint position before and after a posterior glide mobilization. 

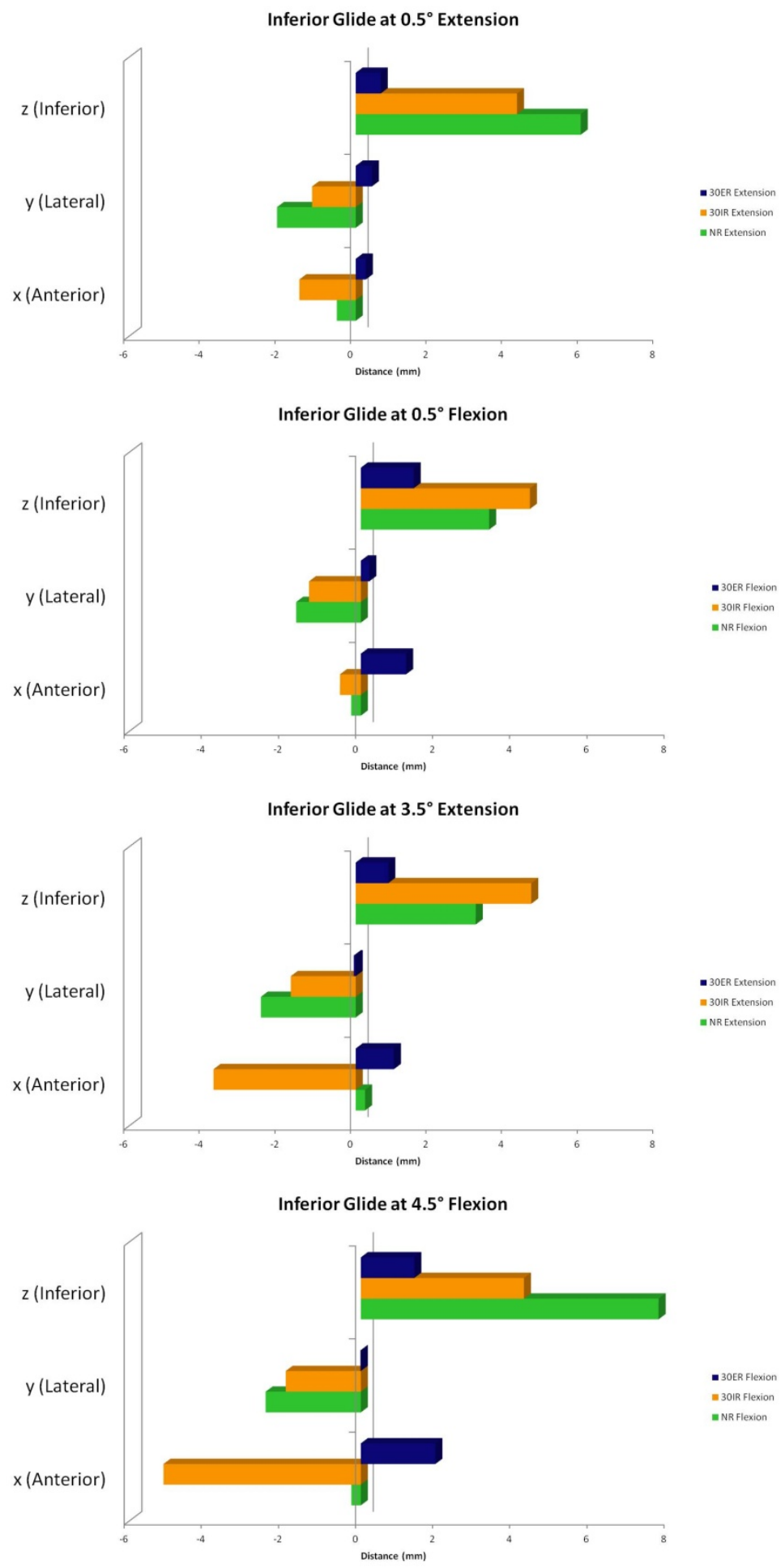

Figure 4-12. Effects of IR/ER and F/E on GH joint position before and after an inferior glide mobilization. 


\section{Inferior Glide}

Thirty degrees of ER combined with extension increased stiffness to a greater extent than flexion in the inferior glide (Figure 4-8). Both flexion and extension produce an inferior glide which may decrease the amount of "slack" present during an inferior glide mobilization produced by the robot (Figure 4-12). Flexion and extension both cause posterior motion in the inferior glide. Flexing the joint also appears to increase inferior motion the most. The reason why stiffness increased to a greater extent than flexion may be because extension produced a smaller displacement inferiorly than flexion. NR inferior glide mobilization produced the least amount of stiffness in flexion, but for extension the stiffness increased at $5^{\circ}$ of extension which may suggest that the humeral head moves to a more inferior position to a greater extent than flexion. The increased stiffness could also be contributed to more soft tissue engagement.

\section{Discussion}

The externally rotated position caused the highest joint stiffness in every glide (Table 4-1). This was expected, because externally rotation the specimen moves the joint towards a closed pack position (Hertling and Kessler, 2006), which naturally stiffens up the joint. Internal rotation caused higher stiffness in the posterior and inferior glides versus the neutral rotation glides. The anterior glide in NR position gave similar stiffness values to the anterior glide in the IR position. IR and ER were investigated in this study as a means of validation the protocol. Based off of the anatomy of the GH joint, the stiffness should increase with IR and increase more so with ER.

McQuade et al. (2012) captured the GH stiffness of a single axis; the current study was able to capture GH 3D stiffness. Comparing only the MOB axes with McQuade's data, similar stiffness results from his in vivo study were similar to the values recorded in this in vitro cadaver study. External and internal humeral rotation stiffness data varied. However, the degrees of humeral rotation were not reported within McQuade's study. Figure 4-13 shows a comparison graph of McQuade's findings along with data in this study. Robotic anterior glide stiffness was found to be within one standard deviation of the published in vivo data. Robotic posterior stiffness was lower than expected when compared to in vivo. Upon reviewing data further, axial force only built up to $15 \mathrm{~N}$, whereas pilot PT data recorded in a previous pilot study was $25 \mathrm{~N}$. In other words, the position control strategies stopped the glide from reaching a higher load, thus reducing the joint stiffness. If allowed to continue past the $20 \mathrm{~mm}$ glide limit, the forces would have been larger, thus increasing the recorded stiffness. This confirms that the programmed protocol works as designed and allows for, not only force control, but also position control. Clinical studies may not always capture the most reliable force and displacement/magnitudes induced by the therapist, as well as joint reactive behaviors (osteokinematics and 3D force/moment reactions due to soft tissue). Because of the nature of the robotic controls, the error associated with the measurements becomes a function of the resolution of the robot itself $( \pm 0.2 \mathrm{~N}$ and $\pm 2 \mu \mathrm{m})$. The algorithm used to balance the forces about the $\mathrm{GH}$ joint after internal and external rotation used a motion 


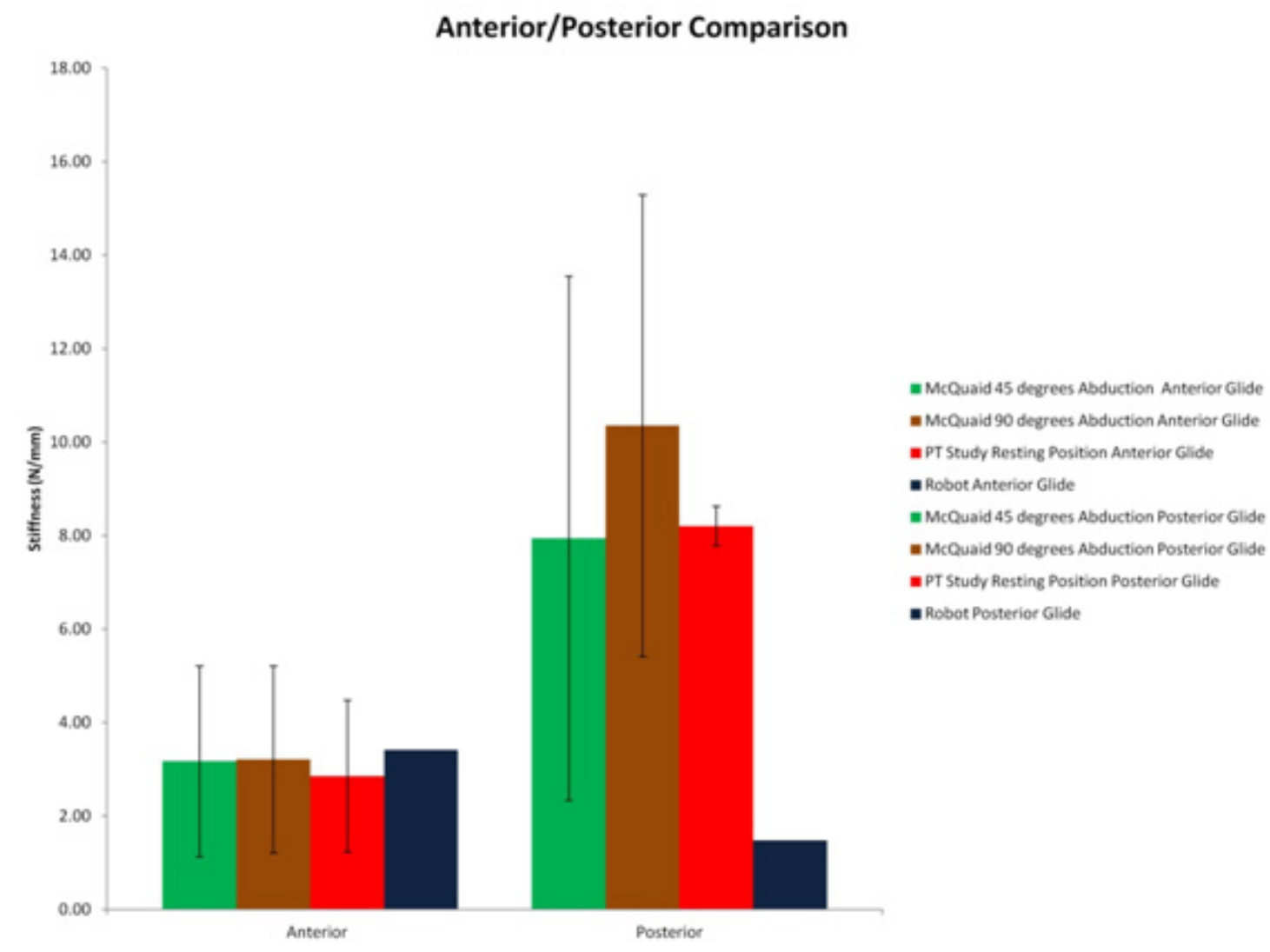

Figure 4-13. In vivo comparison between axial GH stiffness values.

Source: McQuade, K., Price, R., Liu, N., and Ciol, M. "Objective Assessment of Joint Stiffness: A Clinically Oriented Hardware and Software Device with an Application to the Shoulder Joint." Physiotherapy (2012). 
step size of $0.25 \mathrm{~mm}$, which helps to explains why the highest standard deviation of the IR and ER population was $0.5 \mathrm{~mm}$. These shortcomings make cadaver in vitro studies an invaluable tool for understanding the basic joint mechanics of mobilization therapy.

During an actual in vivo mobilization, it is likely that the RC muscles will be dynamic and reactive instead of static. Also, there are many more muscles involved with the shoulder's motion beside the RC muscles simulated in this study. Patients with injured shoulders will sometimes guard their shoulder by using active muscles to keep their joints largely immobile. Patient guarding was not simulated in this study. As with all in vitro testing, in vivo generalizations should be approached with discretion.

The GH joint has $6 \mathrm{DOF}$, but the current UTHSC biorobotic testing platform is only capable of controlling 4DOF and unconstraining a 5th DOF. This, by itself, expands on the work of others who use up to four DOF systems (Hsu and Headman, 2002, Bryce et al., 2010). Other robotic studies have examined the effects of glides, but none have observed theeffects of internal and external rotation coupled with mobilizations. No three dimensional stiffness values for the GH joint have been published to our knowledge (Browe et al., 2011, Voycheck et al., 2011, Hsu and Headman, 2006, Hsu et al., 2000, Hsu and Headman, 2002, Hsu et al., 2002, Wuelker and Wirth, 1995, Karduna et al., 2005 , Bryce et al., 2010). This study only explored a range of $20^{\circ}$ and used a single specimen throughout testing. The GH joint is the most mobile joint in the human body, and a higher ROM with multiple specimens should be studied using this protocol for further biomechanical understanding.

The objective of this protocol was to simulate a glide by applying a force on the specimen. The robot protocol was programmed to induce a prescribed load ( $20 \mathrm{~N}$, based off of a previous PT study, to emulate actual MOB forces) in the appropriate anatomical direction, and then minimize the loads in the controlled off-axis. Forces in the robotic inferior test were programmed to distract the specimen to $15 \mathrm{~N}$ then minimize off axis forces to $\pm 5 \mathrm{~N}$. Inferior load differences show how this protocol can apply a prescribed load to the specimen. This demonstrates the sensitivity of the RTP's balancing routine. The precision of force and displacement this robotic platform can control far exceeds the capacities controllability of any other GH platform to our knowledge.

\section{Conclusion}

A novel robotic testing protocol was developed for studying manual therapy techniques used to treat the GH joint. The test setup and protocol allowed for simulation of Anterior, Posterior and Inferior joint mobilization glides in varying degrees of internal and external rotation with a controllable force input and the ability to apply and minimize off axis forces. For future work, unique loads and distances can be prescribed for simulating other glides and/or activities. Surgical techniques and arthroplasties can be evaluated under prescribed forces for biomechanical simulation. Injury models can also be developed from this protocol to further investigate $\mathrm{GH}$ biomechanics. 


\section{CHAPTER 5. CONCLUSION, LIMITATIONS, AND FUTURE WORK}

A functional protocol was developed for analyzing individual therapist performing manual therapy. This protocol evaluated neutral and resting position manual therapy using grade III non-oscillatory Anterior, Posterior, and Inferior glides. This model would be ideal for developing injury models (by creating a pathology on the specimen), and future work could expand on this idea. In order to more thoroughly evaluate the mechanics of manual articulation, a robotic system was modified to accommodate the shoulder anatomy and a protocol was developed simulate these manual therapies through a volume of space. The simulation was designed around force limits applied by the therapists in the manual articulation study. This protocol successfully glided the GH joint anteriorly, posteriorly, and inferiorly until a force limit within $\pm 5 \mathrm{~N}$ of the PTs applied force is reached.

A novel robotic testing protocol was successfully developed for simulating manual therapy techniques performed by PTs. The ROM was limited in the robotic study compared to the anatomical ROM of the GH joint. Future work for this particular protocol should be aimed at improving the robotic setup/fixturing to increase the studied range of motion, thus allowing for a more thorough evaluation of the joint. Because of the rotator cuff lines and limited DOF of the robot, resting position was not able to be simulated via the robot. Future work can expand on simulation protocols by prescribing unique loads and distances for simulating other glides and/or activities. Surgical techniques and arthroplasties can be evaluated under force or displacement simulation. Injury models can also be developed from this protocol for further $\mathrm{GH}$ biomechanical investigation.

To expand on this research as a whole, in vivo data are needed. Utilizing a gait lab with 3D markers and a force plate, it would be possible to simulate a similar experiment on a human specimen. The described experiment would simulate a truly clinical scenario, and allow for 3D position and force data to be collected in vivo. Utilizing this setup as a model, it could be used to assess therapists as they develop their manual therapy technique and act as a rubric for standardizing manual therapy. A psudomodel of the GH joint, or any joint in the body for that matter, can be developed based off of in vivo data gathered. The experimental setup would resemble the work of McQuade et al., except that measurements could be recorded in three dimensions. 


\section{LIST OF REFERENCES}

Bono, C. M., R. Renard, and A. S. Levy. "Effect of Displacement of Fractures of the Greater Tuberosity on the Mechanics of the Shoulder." The Journal of Bone and Joint Surgery (2001).

Branch, T. P., O. Avilla, L. London, and W. C. Hutton. "Correlation of Medial/lateral Rotation of the Humerus with Glenohumeral Translation." British Journal of Sports Medicine. (1999).

Browe, D., C. A. Voycheck, P. J. McMahon, and R. E. Debski. "Injury to the Glenohumeral Capsule During Anterior Dislocation Results in Damage to the Anteroinferior Capsule." ASME 2011 Summer Bioengineering Conference. (2011).

Brudvig, T, Kulkarni, H, Shah, S. "The Effect of Therapeutic Exercise and Mobilization on Patients With Shoulder Dysfunction: A Systematic Review With Metaanalysis." Journal of Orthopaedic and Sports Physical Therapy. (2011).

Bryce, Chris D., Andrew C. Davison, Nori Okita, Gregory S. Lewis, Neil A. Sharkey, and April D. Armstrong. "A Biomechanical Study of Posterior Glenoid Bone Loss and Humeral Head Translation." Journal of Shoulder and Elbow Surgery (2010).

Bulgen, D., Binder, A, Hazleman, B , Dutton, J, Roberts, S. "Frozen Shoulder: Prospective Clinical Study with an Evaluation of Three Treatment Regimens." Annals of the Rheumatic Diseases. (1984).

Butcher, J., Zukowski C, Brannen S, O'Connor, F, Lillegard, W. "Patient Profile, Referral Sources, and Consultant Utilization in a Primary Care Sports Medicine Clinic." Journal of Family Practice 43 (1996).

Clarkson, H. Musculoskeletal Assessment: Joint Range of Motion and Manual Muscle Strength. Philadelphia: Lippincott Williams \& Wilkins, 2000.

Donatelli R, Greenfield B. Case study: rehabilitation of a stiff and painful shoulder: a biomechanical approach. Journal of Orthopaedic and Sports Physical Therapy. (1987).

Farrell J , Gensen G. Manual Therapy: A Critical Assessment of Role in the Profession of Physical Therapy. Physical Therapy (1992).

Fongemie, A., Buss, D., and Rolnick, S. "Management of Shoulder Impingement Syndrome and Rotator Cuff Tears." American Family Physician (1998).

Gray, H, Pickering, Pick T, Howden R. Gray's Anatomy. Philadelphia: Courage, (1974). 
Green S, Buchbinder R, Hetrick S. Physiotherapy interventions for shoulder pain. Cochrane Database Systematic Review. (2003).

Hertling, D and Kessler, R. Management of Common Musculoskeletal Disorders. Lippincott Philadelphia, 4th edition. (2006).

Ho, K, and Hsu A. "Displacement of the Head of Humerus While Performing "Mobilization with Movements" in Glenohumeral Joint: A Cadaver Study." Manual Therapy. (2009).

Hsu A, Chiu J, and Chang J. "Biomechanical Analysis of Axial Distraction Mobilization of the Glenohumeral Joint - A Cadaver Study." Manual Therapy. (2009).

Hsu, A, and Headman T. "Changes in Abduction and Rotation Range of Motion in Response to Simulated Dorsal and Ventral Translational Mobilization of the Glenohumeral Joint." Journal of the American Physical Therapy Association. (2002).

Hsu, A, Ho, L, Ho, S, Headman, T. "Joint Position during Anterior-posterior Glide Mobilization: Its Effect on Glenohumeral Abduction Range of Motion." Archives of Physical Medicine and Rehabilitation. (2000).

Hsu, A., Ho L, Chang J, Chang G. "Characterization of Tissue Resistance during a Dorsally Directed Translational Mobilization of the Glenohumeral Joint." Archives of Physical Medicine and Rehabilitation. (2002).

Hsu, A., Ho L, Ho S, Headman T. "Immediate Response of Glenohumeral Abduction Range of Motion to a Caudally Directed Translational Mobilization: A Fresh Cadaver Simulation." Archives of Physical Medicine and Rehabilitation. (2000).

Johnson A, Godges, J, Zimmerman G, Ounanian L. "The Effect of Anterior Versus Posterior Glide Joint Mobilization on External Rotation Range of Motion in Patients With Shoulder Adhesive Capsulitis." Journal of Orthopaedic and Sports Physical Therapy. (2007).

Karduna, A., Kerner P, Lazarus M. "Contact Forces in the Subacromial Space: Effects of Scapular Orientation." Journal of Shoulder and Elbow Surgery. (2005).

Kelley, M McClure P, Leggin P. "Frozen Shoulder: Evidence and a Proposed Model Guiding Rehabilitation." Journal of Orthopaedic and Sports Physical Therapy. (2009). 
Kelly, B and DiAngelo D. "A Multi-axis Programmable Spine Robot for the Study of Multi-body Spinal Biomechanics Using Real-time Hybrid Force and Displacement Control Strategies." University of Tennessee Health Science Center. (2006).

Krackow, K, Thomas S, and Jones L. "A New Stitch for Ligament-tendon Fixation. Brief Note." The Journal of Bone and Joint Surgery. (2011).

Lin, H., A. Hsu, K. An, J. Changchien, T. Kuan, and G. Chang. "Reliability of Stiffness Measured in Glenohumeral Joint and Its Application to Assess the Effect of Endrange Mobilization in Subjects with Adhesive Capsulitis." Manual Therapy. (2008).

Mahaffey, B., Smith P. "Shoulder Instability in Young Athletes." American Family Physician (1999).

Maitland G. Peripheral Manipulation. London: Butterworths. (1977).

McQuade, K., R. Price, N. Liu, and M. Ciol. Objective Assessment of Joint Stiffness: A Clinically Oriented Hardware and Software Device with an Application to the Shoulder Joint. Physiotherapy (2012).

Nicholson, G. "The Effects of Passive Joint Mobilization on Pain and Hypomobility Associated with Adhesive Capsulitis of the Shoulder." Journal of Orthopaedic and Sports Physical Therapy. (1985).

Palmer, L. and M. E. Epler. Clinical Assessment Procedures in Physical Therapy. Lippincott Williams \& Wilkins. (1990).

Rohen, J., Yokochi C. Color atlas of anatomy : a photographic study of the human body. Baltimore, MD, Lippincott Williams \& Wilkins (2002). (http://lww.com).

Rollins, C A, and Robinson J L. "Evaluation of Undergraduate Physical Therapy Students' Comprehension of Maitland's Grades (I-IV) for Posterior Mobilization of the Glenohumeral Joint." Journal of Orthopaedic and Sports Physical Therapy. (1980).

Van der Windt D, Bouter L. Physiotherapy or corticosteroid injection for shoulder pain. Annals of the Rheumatic Diseases. (2003).

Vermeulen H, Obermann W, Burger B, Kok G, Rozing P, van Den Ende C. End-range mobilization techniques in adhesive capsulitis of the shoulder joint: A multiplesubject case report. Physical Therapy. (2000). 
Vermeulen, H., P. Rozing, W. Obermann, S. Cessie, and T. Vileland. "Comparison of High-Grade and Low-Grade Mobilization Techniques in the Management of Adhesive Capsulitis of the Shoulder: Randomized Controlled Trial." Physical Therapy. (2006).

Voycheck, C., Browe D, McMahon P, Debski R. "Injury to the Glenohumeral Capsule During Anterior Dislocation Leads to Higher Joint Contact Forces During Simulated Clinical Exams." ASME 2011 Summer Bioengineering Conference (2011).

Wilk K, Reinold M, Macrina L, Porterfield R, Devine K, Suarez K, and Andrews J. "Glenohumeral Internal Rotation Measurements Differ Depending on Stabilization Techniques." Sports Health: A Multidisciplinary Approach. (2009).

Wuelker, N., Wirth C. "A Dynamic Shoulder Model: Reliability Testing and Muscle Force Study." Journal of Biomechanics 28 (1995).

Yiasemides, Halaki R, Ginn K. "Does Passive Mobilization of Shoulder Region Joints Provide Additional Benefit Over Advice and Exercise Alone for People Who Have Shoulder Pain and Minimal Movement Restriction? A Randomized Controlled Trial." Physical Therapy. (2011). 


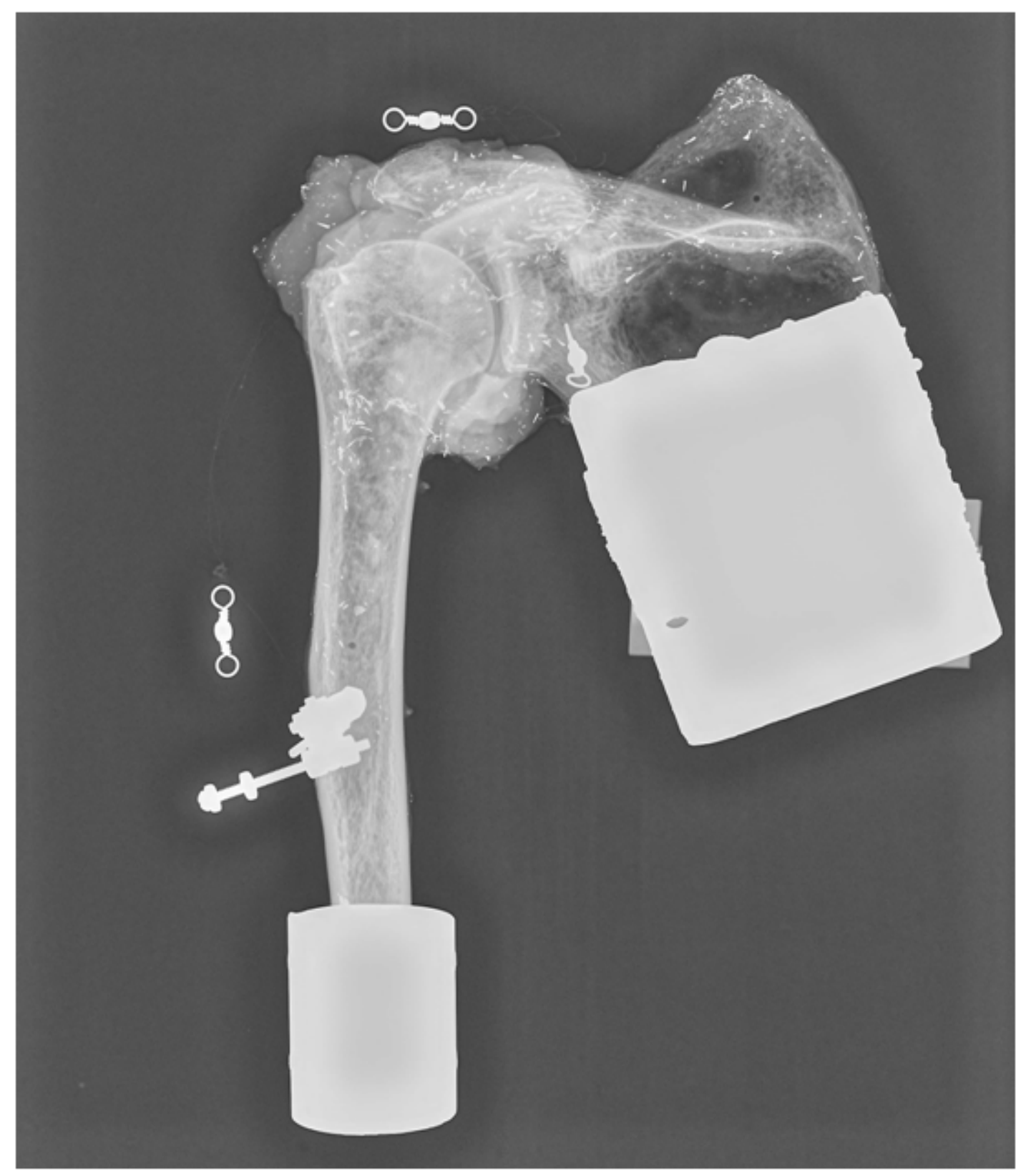

Figure A-1. Manual articulation specimen.

Specimen has been potted, sutured and instrumented with a rod for 3D target placement. 


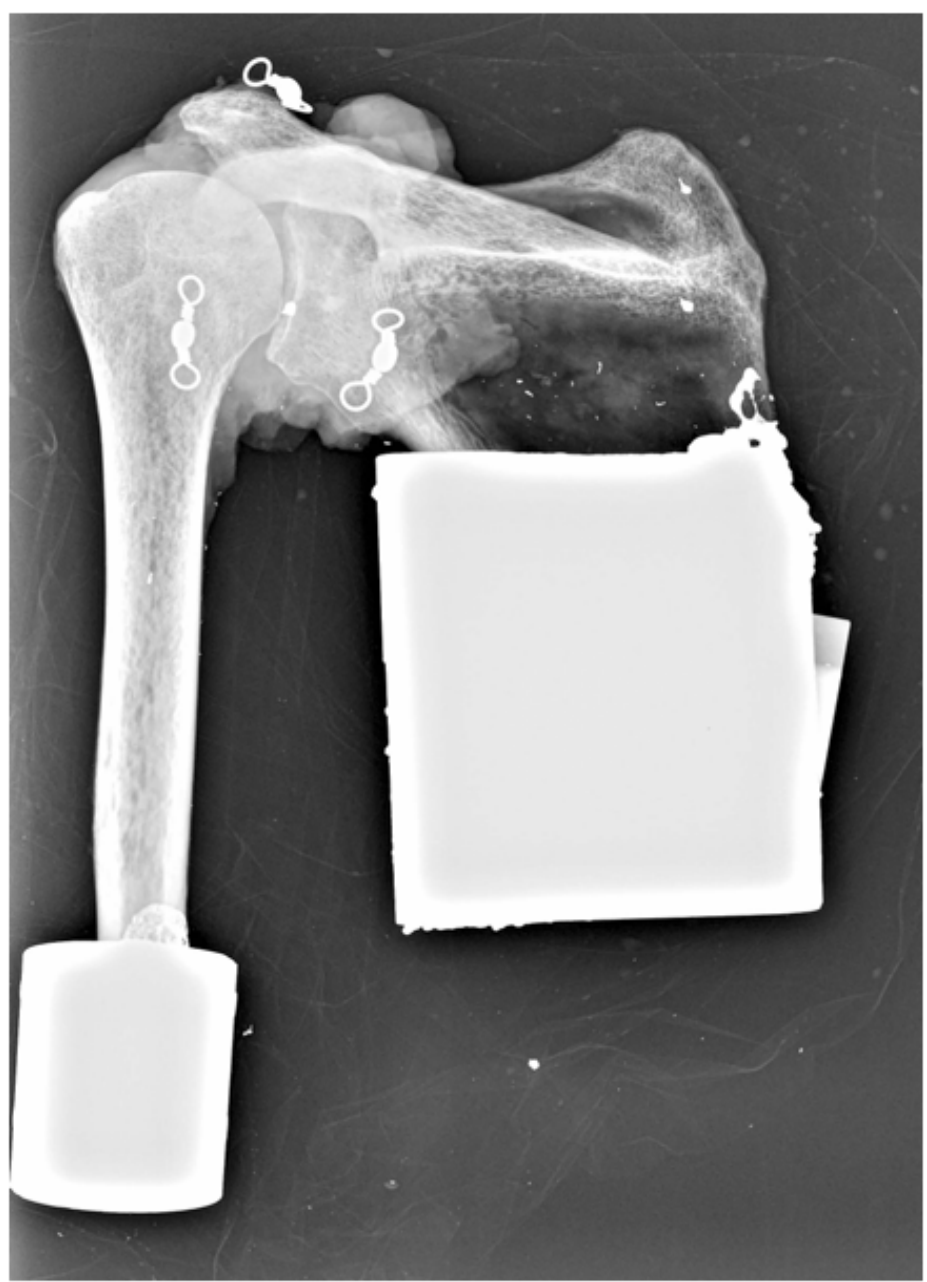

Figure A-2. Robot specimen.

Specimen has been potted and sutured. 


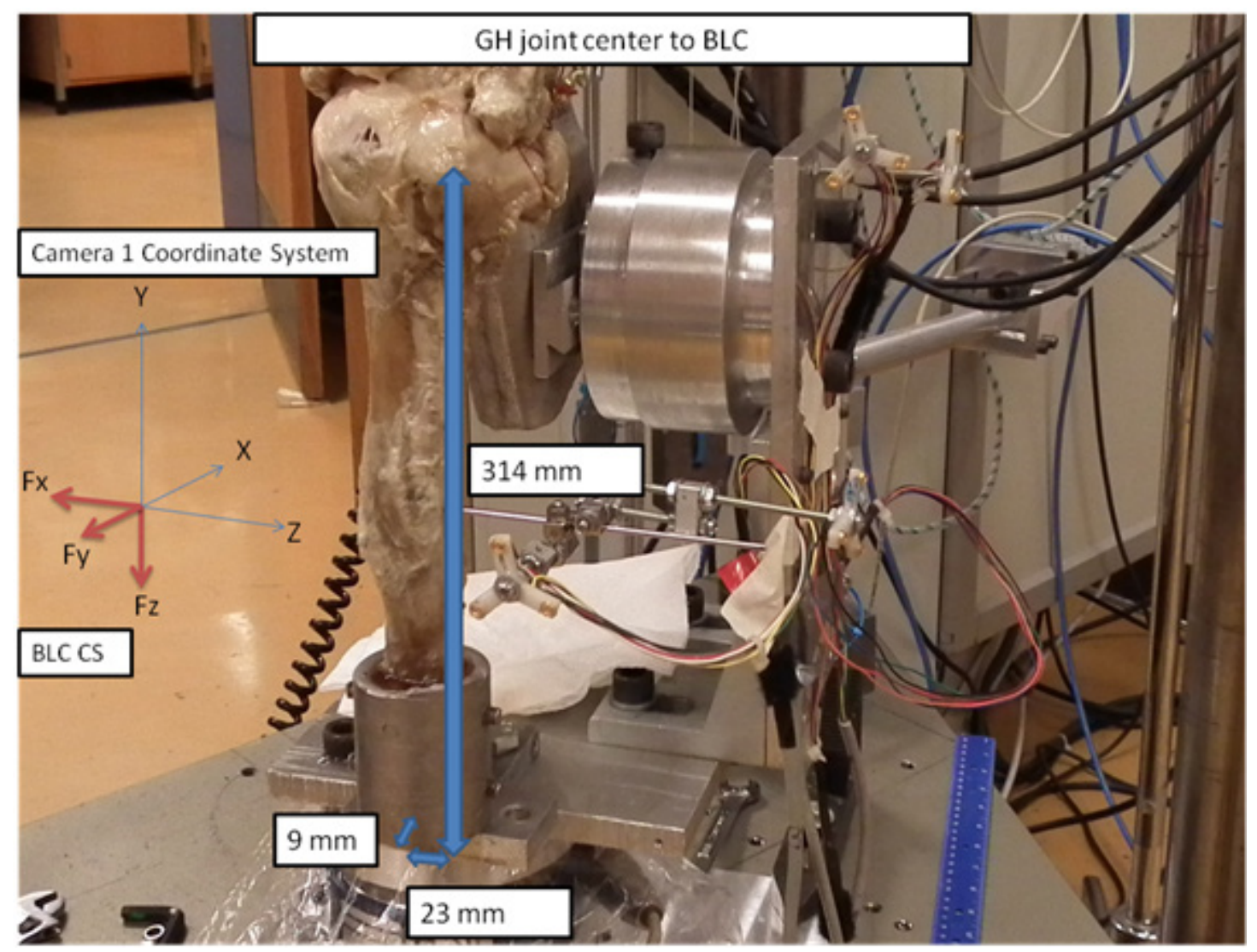

Figure A-3. Manual articulation specimen with force frame offsets. 


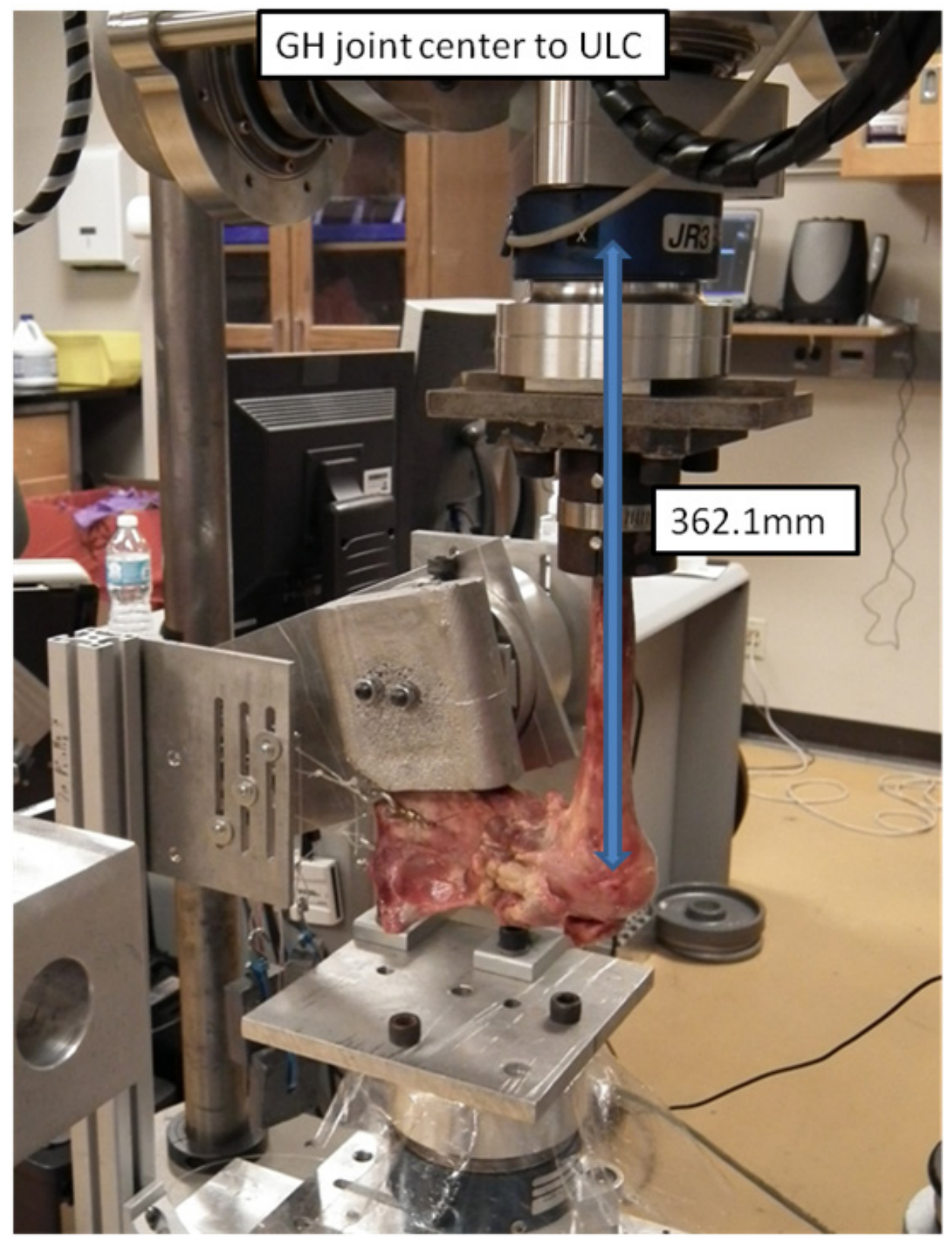

Figure A-4. Robot specimen with force frame offsets. 


\section{APPENDIX B: PT PROTOCOL DATASET}

Table B-1. Applied PT forces.

\begin{tabular}{|c|c|c|c|c|c|c|}
\hline Glide & Therapist & Run & X Force & Y Force & Z Force & 3D Force \\
\hline \multirow{6}{*}{$\begin{array}{l}\text { Anterior Glide } \\
\text { Resting Position }\end{array}$} & \multirow{3}{*}{ PT 1} & 1 & 14.26 & -0.64 & 16.7 & 21.97 \\
\hline & & 2 & 14.26 & 1.4 & 21.19 & 25.57 \\
\hline & & 3 & 11.82 & 3.03 & 22.41 & 25.51 \\
\hline & \multirow{3}{*}{ PT 2} & 1 & 20.37 & -8.38 & 19.56 & 29.45 \\
\hline & & 2 & 21.19 & -11.23 & 18.33 & 30.18 \\
\hline & & 3 & 22 & -10.42 & 18.74 & 30.72 \\
\hline \multirow{6}{*}{$\begin{array}{l}\text { Anterior Glide } \\
\text { Neutral Position }\end{array}$} & \multirow{3}{*}{ PT 1} & 1 & 2.04 & 21.36 & 22.82 & 31.32 \\
\hline & & 2 & 0 & 18.1 & 23.22 & 29.44 \\
\hline & & 3 & -0.41 & 23.4 & 28.52 & 36.89 \\
\hline & \multirow{3}{*}{ PT 2} & 1 & 13.04 & 12.4 & 12.63 & 21.98 \\
\hline & & 2 & 15.89 & 16.07 & 15.48 & 27.39 \\
\hline & & 3 & 13.85 & 14.44 & 17.52 & 26.59 \\
\hline \multirow{6}{*}{$\begin{array}{l}\text { Posterior Glide } \\
\text { Resting Position }\end{array}$} & \multirow{3}{*}{ PT 1} & 1 & -2.04 & 3.44 & -29.74 & 30.01 \\
\hline & & 2 & -3.67 & 4.25 & -29.74 & 30.27 \\
\hline & & 3 & -4.07 & 5.47 & -30.56 & 31.31 \\
\hline & \multirow{3}{*}{ PT 2} & 1 & -26.89 & -5.93 & -33.82 & 43.61 \\
\hline & & 2 & -25.26 & -0.23 & -34.22 & 42.54 \\
\hline & & 3 & -22 & 3.03 & -37.89 & 43.92 \\
\hline \multirow{6}{*}{$\begin{array}{l}\text { Posterior Glide } \\
\text { Neutral Position }\end{array}$} & \multirow{3}{*}{ PT 1} & 1 & 3.67 & -10.42 & -19.96 & 22.81 \\
\hline & & 2 & 0.41 & -9.6 & -19.96 & 22.16 \\
\hline & & 3 & 3.67 & -21.42 & -18.74 & 28.69 \\
\hline & \multirow{3}{*}{ PT 2} & 1 & -0.82 & -4.71 & -23.22 & 23.71 \\
\hline & & 2 & 4.07 & -1.86 & -20.37 & 20.86 \\
\hline & & 3 & -2.45 & 0.18 & -24.44 & 24.57 \\
\hline \multirow{6}{*}{$\begin{array}{l}\text { Inferior Glide } \\
\text { Resting Position }\end{array}$} & \multirow{3}{*}{ PT 1} & 1 & 24.44 & -28.34 & 3.26 & 37.57 \\
\hline & & 2 & 18.33 & -24.27 & 4.48 & 30.74 \\
\hline & & 3 & 22.81 & -31.6 & 2.04 & 39.03 \\
\hline & \multirow{3}{*}{ PT 2} & 1 & -15.48 & -24.27 & -3.67 & 29.02 \\
\hline & & 2 & -14.26 & -27.93 & -4.89 & 31.74 \\
\hline & & 3 & -11 & -26.3 & -3.26 & 28.7 \\
\hline \multirow{6}{*}{$\begin{array}{l}\text { Inferior Glide } \\
\text { Neutral Position }\end{array}$} & \multirow{3}{*}{ PT 1} & 1 & -1.22 & -27.53 & 0.41 & 27.56 \\
\hline & & 2 & -1.63 & -27.53 & 0.41 & 27.58 \\
\hline & & 3 & 2.04 & -27.12 & -0.82 & 27.21 \\
\hline & \multirow{3}{*}{ PT 2} & 1 & 6.52 & -28.34 & 2.85 & 19.67 \\
\hline & & 2 & 8.96 & -27.12 & 3.67 & 19.66 \\
\hline & & 3 & 4.08 & -27.12 & 1.63 & 17.67 \\
\hline
\end{tabular}

All forces are in Newton. 
Table B-2. Applied PT displacements.

\begin{tabular}{|c|c|c|c|c|c|c|}
\hline Glide & Therapist & Run & X Position & Y Position & Z Position & 3D Position \\
\hline \multirow{6}{*}{$\begin{array}{l}\text { Anterior Glide } \\
\text { Resting Position }\end{array}$} & \multirow{3}{*}{ PT 1} & 1 & 1.28 & -4.2 & 6.5 & 7.84 \\
\hline & & 2 & 5.26 & 11.56 & 4.92 & 13.61 \\
\hline & & 3 & -4.59 & 11.99 & 5 & 13.78 \\
\hline & \multirow{3}{*}{ PT 2} & 1 & 8.78 & 0.05 & 12.53 & 15.3 \\
\hline & & 2 & 7.08 & 0.52 & 14.31 & 15.97 \\
\hline & & 3 & 6.69 & 1.56 & 14.23 & 15.81 \\
\hline \multirow{6}{*}{$\begin{array}{l}\text { Anterior Glide } \\
\text { Neutral Position }\end{array}$} & \multirow{3}{*}{ PT 1} & 1 & -0.94 & -12.46 & 12.97 & 18.01 \\
\hline & & 2 & -6.79 & -12.8 & 15.78 & 21.42 \\
\hline & & 3 & 2.39 & -14.9 & 19.14 & 24.37 \\
\hline & \multirow{3}{*}{ PT 2} & 1 & 1.99 & 9.56 & 9.97 & 13.96 \\
\hline & & 2 & 7.6 & 13.45 & 10.02 & 18.41 \\
\hline & & 3 & 10.77 & 11.82 & 10.43 & 19.09 \\
\hline \multirow{6}{*}{$\begin{array}{l}\text { Posterior Glide } \\
\text { Resting Position }\end{array}$} & \multirow{3}{*}{ PT 1} & 1 & -1.29 & 2.8 & -6.92 & 7.58 \\
\hline & & 2 & -1.18 & 4.28 & -8.19 & 9.32 \\
\hline & & 3 & 2.03 & 2.71 & -10.29 & 10.84 \\
\hline & \multirow{3}{*}{ PT 2} & 1 & 1.35 & 2.12 & -4.56 & 5.2 \\
\hline & & 2 & 3.31 & -6.02 & -3.87 & 7.89 \\
\hline & & 3 & 4.2 & -2.67 & -4.68 & 6.83 \\
\hline \multirow{6}{*}{$\begin{array}{l}\text { Posterior Glide } \\
\text { Neutral Position }\end{array}$} & \multirow{3}{*}{ PT 1} & 1 & 1.43 & 8.29 & -9.73 & 12.86 \\
\hline & & 2 & 5.63 & 5.02 & -12.2 & 14.35 \\
\hline & & 3 & 9.9 & 7.46 & -10.74 & 16.4 \\
\hline & \multirow{3}{*}{ PT 2} & 1 & -16.57 & -6.5 & -14.88 & 23.2 \\
\hline & & 2 & -8.58 & -5.64 & -11.68 & 15.55 \\
\hline & & 3 & -9.66 & -8.53 & -15.1 & 19.85 \\
\hline \multirow{6}{*}{$\begin{array}{l}\text { Inferior Glide } \\
\text { Resting Position }\end{array}$} & \multirow{3}{*}{ PT 1} & 1 & -13.39 & -18.22 & -6.09 & 23.42 \\
\hline & & 2 & -22.66 & -10.4 & -11.37 & 27.4 \\
\hline & & 3 & -22.24 & -12.86 & -13.04 & 28.81 \\
\hline & \multirow{3}{*}{ PT 2} & 1 & 3.23 & -27.81 & 6.28 & 28.69 \\
\hline & & 2 & 8.21 & -31.99 & 9.92 & 34.49 \\
\hline & & 3 & 5.56 & -26.27 & 8.09 & 28.05 \\
\hline \multirow{6}{*}{$\begin{array}{l}\text { Inferior Glide } \\
\text { Neutral Position }\end{array}$} & \multirow{3}{*}{ PT 1} & 1 & -9.12 & -14.58 & -2.15 & 17.33 \\
\hline & & 2 & -13.51 & -19.27 & -3.93 & 23.86 \\
\hline & & 3 & -10.63 & -19.33 & -1.53 & 22.11 \\
\hline & \multirow{3}{*}{ PT 2} & 1 & -6.22 & -18.94 & -12.18 & 23.36 \\
\hline & & 2 & -6.99 & -16.32 & -7.53 & 19.28 \\
\hline & & 3 & -2.6 & -14.49 & -4.06 & 15.27 \\
\hline
\end{tabular}

All distances are in millimeters. 
Table B-3. Calculated PT stiffness.

\begin{tabular}{|c|c|c|c|c|c|c|}
\hline Glide & Therapist & Run & X Stiffness & Y Stiffness & Z Stiffness & 3D Stiffness \\
\hline \multirow{6}{*}{$\begin{array}{l}\text { Anterior Glide } \\
\text { Resting Position }\end{array}$} & \multirow{3}{*}{ PT 1} & 1 & 11.16 & 0.15 & 2.57 & 2.8 \\
\hline & & 2 & 2.71 & 0.12 & 4.31 & 1.88 \\
\hline & & 3 & -2.58 & 0.25 & 4.48 & 1.85 \\
\hline & \multirow{3}{*}{ PT 2} & 1 & 2.32 & -160.76 & 1.56 & 1.92 \\
\hline & & 2 & 2.99 & -21.54 & 1.28 & 1.89 \\
\hline & & 3 & 3.29 & -6.68 & 1.32 & 1.94 \\
\hline \multirow{6}{*}{$\begin{array}{l}\text { Anterior Glide } \\
\text { Neutral Position }\end{array}$} & \multirow{3}{*}{ PT 1} & 1 & -2.17 & -1.71 & 1.76 & 1.74 \\
\hline & & 2 & 0 & -1.41 & 1.47 & 1.37 \\
\hline & & 3 & -0.17 & -1.57 & 1.49 & 1.51 \\
\hline & \multirow{3}{*}{ PT 2} & 1 & 6.56 & 1.3 & 1.27 & 1.58 \\
\hline & & 2 & 2.09 & 1.19 & 1.55 & 1.49 \\
\hline & & 3 & 1.29 & 1.22 & 1.68 & 1.39 \\
\hline \multirow{6}{*}{$\begin{array}{l}\text { Posterior Glide } \\
\text { Resting Position }\end{array}$} & \multirow{3}{*}{ PT 1} & 1 & 1.58 & 1.23 & 4.3 & 3.96 \\
\hline & & 2 & 3.09 & 0.99 & 3.63 & 3.25 \\
\hline & & 3 & -2.01 & 2.02 & 2.97 & 2.89 \\
\hline & \multirow{3}{*}{ PT 2} & 1 & -19.88 & -2.8 & 7.42 & 8.38 \\
\hline & & 2 & -7.63 & 0.04 & 8.84 & 5.39 \\
\hline & & 3 & -5.24 & -1.13 & 8.09 & 6.43 \\
\hline \multirow{6}{*}{$\begin{array}{l}\text { Posterior Glide } \\
\text { Neutral Position }\end{array}$} & \multirow{3}{*}{ PT 1} & 1 & 2.57 & -1.26 & 2.05 & 1.77 \\
\hline & & 2 & 0.07 & -1.91 & 1.64 & 1.54 \\
\hline & & 3 & 0.37 & -2.87 & 1.74 & 1.75 \\
\hline & \multirow{3}{*}{ PT 2} & 1 & 0.05 & 0.73 & 1.56 & 1.02 \\
\hline & & 2 & -0.47 & 0.33 & 1.74 & 1.34 \\
\hline & & 3 & 0.25 & -0.02 & 1.62 & 1.24 \\
\hline \multirow{6}{*}{$\begin{array}{l}\text { Inferior Glide } \\
\text { Resting Position }\end{array}$} & \multirow{3}{*}{ PT 1} & 1 & -1.83 & 1.56 & -0.54 & 1.6 \\
\hline & & 2 & -0.81 & 2.33 & -0.39 & 1.12 \\
\hline & & 3 & -1.03 & 2.46 & -0.16 & 1.35 \\
\hline & \multirow{3}{*}{ PT 2} & 1 & -4.79 & 0.87 & -0.58 & 1.01 \\
\hline & & 2 & -1.74 & 0.87 & -0.49 & 0.92 \\
\hline & & 3 & -1.98 & 1 & -0.4 & 1.02 \\
\hline \multirow{6}{*}{$\begin{array}{l}\text { Inferior Glide } \\
\text { Neutral Position }\end{array}$} & \multirow{3}{*}{ PT 1} & 1 & 0.13 & 1.89 & -0.19 & 1.59 \\
\hline & & 2 & 0.12 & 1.43 & -0.1 & 1.16 \\
\hline & & 3 & -0.19 & 1.4 & 0.53 & 1.23 \\
\hline & \multirow{3}{*}{ PT 2} & 1 & -1.05 & 1.5 & -0.23 & 0.84 \\
\hline & & 2 & -1.28 & 1.66 & -0.49 & 1.02 \\
\hline & & 3 & -1.57 & 1.87 & -0.4 & 1.16 \\
\hline
\end{tabular}

All stiffnesses are in Newton per millimeter. 
APPENDIX C: ROBOTIC PROTOCOL DATASET

Table C-1. Anterior glide NR flexion.

\begin{tabular}{ccccccccc}
\hline Normalized Pitch & $\mathbf{x}$ & $\mathbf{y}$ & $\mathbf{z}$ & $\mathbf{F x}$ & $\mathbf{F y}$ & $\mathbf{F z}$ & 3D Position & 3D Force \\
\hline 0.50 & 8.07 & -0.65 & 0.25 & -28.40 & 7.30 & -3.43 & 8.10 & 29.52 \\
1.00 & 8.47 & -0.64 & 0.50 & -28.08 & 8.48 & -2.89 & 8.51 & 29.47 \\
1.50 & 8.78 & -0.71 & 0.50 & -29.51 & 8.66 & -4.40 & 8.82 & 31.06 \\
2.00 & 9.09 & -0.73 & 0.50 & -30.96 & 8.96 & -5.71 & 9.13 & 32.74 \\
2.50 & 9.24 & -0.75 & 0.50 & -32.32 & 8.97 & -8.01 & 9.28 & 34.48 \\
3.00 & 9.35 & -0.76 & 0.75 & -30.48 & 9.36 & -4.30 & 9.41 & 32.17 \\
3.50 & 9.47 & -0.70 & 0.75 & -31.31 & 9.23 & -6.81 & 9.53 & 33.34 \\
4.00 & 9.60 & -0.73 & 0.75 & -31.83 & 9.49 & -6.99 & 9.66 & 33.94 \\
4.50 & 9.68 & -0.77 & 1.00 & -30.23 & 9.45 & -5.62 & 9.76 & 32.16 \\
5.00 & 9.78 & -0.72 & 1.00 & -31.00 & 9.53 & -7.49 & 9.85 & 33.29 \\
5.50 & 9.87 & -0.75 & 1.00 & -31.22 & 9.75 & -7.12 & 9.95 & 33.47 \\
6.00 & 9.97 & -0.78 & 0.99 & -31.56 & 9.53 & -9.42 & 10.05 & 34.28 \\
6.50 & 10.07 & -0.85 & 1.24 & -30.23 & 9.49 & -7.44 & 10.19 & 32.55 \\
7.00 & 10.07 & -0.79 & 1.24 & -30.01 & 9.62 & -8.26 & 10.18 & 32.58 \\
7.50 & 10.13 & -0.79 & 1.24 & -30.01 & 9.71 & -7.25 & 10.23 & 32.37 \\
8.00 & 10.12 & -0.81 & 1.24 & -29.64 & 9.53 & -8.44 & 10.22 & 32.26 \\
8.50 & 10.15 & 0.00 & 1.24 & -29.76 & 9.36 & -9.83 & 10.22 & 32.70 \\
9.00 & 10.17 & 0.00 & 1.23 & -29.99 & 9.58 & -10.58 & 10.24 & 33.21 \\
9.50 & 10.13 & 0.00 & 1.23 & -29.64 & 9.62 & -10.76 & 10.20 & 32.97 \\
10.00 & 10.17 & -0.94 & 1.23 & -30.15 & 9.66 & -11.92 & 10.29 & 33.83
\end{tabular}

$\mathrm{x}$ : absolute position of the specimen in the X-axis (mm); $\mathrm{y}$ : absolute position of the specimen in the $\mathrm{Y}$ - axis (mm); $\mathrm{z}$ : absolute position of the specimen in the Z- axis (mm); Fx: absolute forces on the specimen in the X-axis; Fy: absolute forces on the specimen in the Yaxis; Fz: absolute forces on the specimen in the Z-axis. 
Table C-2. Anterior glide NR extension.

\begin{tabular}{ccccccccc}
\hline Normalized Pitch & $\mathbf{x}$ & $\mathbf{y}$ & $\mathbf{z}$ & $\mathbf{F x}$ & $\mathbf{F y}$ & $\mathbf{F z}$ & 3D Position & 3D Force \\
\hline-0.40 & 8.09 & -0.69 & 0.25 & -27.59 & 7.35 & -5.19 & 8.12 & 29.01 \\
-0.90 & 8.35 & -0.58 & 0.25 & -28.56 & 7.57 & -5.85 & 8.38 & 30.12 \\
-1.40 & 8.72 & -0.73 & 0.25 & -31.27 & 7.61 & -6.76 & 8.76 & 32.88 \\
-1.90 & 9.13 & -0.65 & 0.50 & -30.84 & 7.00 & -3.83 & 9.16 & 31.86 \\
-2.40 & 9.30 & -0.64 & 0.50 & -31.41 & 6.30 & -4.77 & 9.34 & 32.38 \\
-2.90 & 9.13 & -0.64 & 0.75 & -27.96 & 6.78 & -1.26 & 9.19 & 28.79 \\
-3.40 & 8.67 & -0.60 & 0.75 & -25.60 & 7.08 & 0.51 & 8.73 & 26.56 \\
-3.90 & 8.57 & -0.59 & 0.75 & -25.70 & 6.21 & -0.22 & 8.62 & 26.44 \\
-4.40 & 8.10 & -0.59 & 0.75 & -23.09 & 6.56 & 0.86 & 8.16 & 24.02 \\
-4.90 & 7.85 & -0.53 & 0.75 & -22.90 & 6.60 & 0.65 & 7.91 & 23.84 \\
-5.40 & 7.69 & -0.48 & 0.75 & -23.20 & 6.73 & -0.09 & 7.74 & 24.16 \\
-5.90 & 7.53 & -0.46 & 0.75 & -23.56 & 6.38 & -0.38 & 7.58 & 24.41 \\
-6.40 & 7.36 & -0.43 & 0.74 & -23.65 & 6.39 & -0.96 & 7.41 & 24.51 \\
-6.90 & 7.30 & -0.43 & 0.99 & -23.20 & 6.52 & 3.03 & 7.38 & 24.29 \\
-7.40 & 7.10 & -0.45 & 0.99 & -23.33 & 6.69 & 1.35 & 7.19 & 24.31 \\
-7.90 & 7.03 & -0.41 & 0.99 & -23.99 & 6.87 & 0.81 & 7.11 & 24.97 \\
-8.40 & 6.88 & -0.36 & 0.99 & -23.85 & 6.43 & 1.62 & 6.96 & 24.76 \\
-8.90 & 6.75 & -0.35 & 0.99 & -24.55 & 6.34 & 0.08 & 6.83 & 25.36 \\
\hline
\end{tabular}

$\mathrm{x}$ : absolute position of the specimen in the X-axis (mm); $\mathrm{y}$ : absolute position of the specimen in the $\mathrm{Y}$ - axis (mm); $\mathrm{z}$ : absolute position of the specimen in the Z- axis (mm); Fx: absolute forces on the specimen in the X-axis; Fy: absolute forces on the specimen in the Yaxis; Fz: absolute forces on the specimen in the Z-axis. 
Table C-3. Anterior glide 30 degrees IR flexion.

\begin{tabular}{ccccccccc}
\hline Normalized Pitch & $\mathbf{x}$ & $\mathbf{y}$ & $\mathbf{z}$ & $\mathbf{F x}$ & $\mathbf{F y}$ & $\mathbf{F z}$ & 3D Position & 3D Force \\
\hline 0.50 & 7.17 & -0.33 & 0.25 & -22.08 & 6.50 & -3.70 & 7.18 & 23.31 \\
1.00 & 7.84 & -0.34 & 0.25 & -24.81 & 6.84 & -5.03 & 7.85 & 26.22 \\
1.50 & 8.28 & -0.41 & 0.25 & -26.91 & 7.25 & -6.87 & 8.29 & 28.70 \\
2.00 & 8.64 & -0.34 & 0.50 & -26.84 & 7.22 & -3.30 & 8.67 & 27.99 \\
2.50 & 8.92 & -0.33 & 0.50 & -27.77 & 6.89 & -4.45 & 8.94 & 28.96 \\
3.00 & 9.30 & -0.31 & 0.50 & -29.76 & 7.35 & -6.46 & 9.32 & 31.32 \\
3.50 & 9.53 & -0.34 & 0.50 & -30.80 & 6.61 & -6.34 & 9.55 & 32.14 \\
4.00 & 9.87 & -0.33 & 0.50 & -32.71 & 6.72 & -8.85 & 9.88 & 34.54 \\
4.50 & 10.00 & -1.52 & 0.75 & -31.10 & 6.05 & -3.75 & 10.14 & 31.90 \\
\hline
\end{tabular}

$\mathrm{x}$ : absolute position of the specimen in the $\mathrm{X}$ - axis $(\mathrm{mm})$; $\mathrm{y}$ : absolute position of the specimen in the $\mathrm{Y}$ - axis (mm); $\mathrm{z}$ : absolute position of the specimen in the Z- axis (mm); Fx: absolute forces on the specimen in the X-axis; Fy: absolute forces on the specimen in the Yaxis; Fz: absolute forces on the specimen in the Z-axis. 
Table C-4. Anterior glide 30 degrees IR extension.

\begin{tabular}{ccccccccc}
\hline Normalized Pitch & $\mathbf{x}$ & $\mathbf{y}$ & $\mathbf{z}$ & $\mathbf{F x}$ & $\mathbf{F y}$ & $\mathbf{F z}$ & 3D Position & 3D Force \\
\hline-0.40 & 7.13 & -0.32 & 0.25 & -18.80 & 7.10 & -4.07 & 7.14 & 20.51 \\
-0.90 & 7.52 & -0.34 & 0.25 & -20.86 & 7.05 & -5.31 & 7.53 & 22.65 \\
-1.40 & 7.90 & -0.35 & 0.25 & -22.34 & 6.84 & -7.02 & 7.91 & 24.39 \\
-1.90 & 8.01 & -0.33 & 0.50 & -21.79 & 5.94 & -3.57 & 8.03 & 22.87 \\
-2.40 & 8.31 & -0.35 & 0.50 & -23.40 & 5.09 & -5.19 & 8.34 & 24.51 \\
-2.90 & 8.54 & -1.05 & 0.50 & -25.01 & 3.85 & -5.61 & 8.62 & 25.91 \\
\hline
\end{tabular}

$\mathrm{x}$ : absolute position of the specimen in the $\mathrm{X}$ - axis $(\mathrm{mm})$; $\mathrm{y}$ : absolute position of the specimen in the $\mathrm{Y}$ - axis (mm); $\mathrm{z}$ : absolute position of the specimen in the Z- axis (mm); Fx: absolute forces on the specimen in the X-axis; Fy: absolute forces on the specimen in the Yaxis; Fz: absolute forces on the specimen in the Z-axis. 
Table C-5. Anterior glide 30 degrees ER flexion.

\begin{tabular}{ccccccccc}
\hline Normalized Pitch & $\mathbf{x}$ & $\mathbf{y}$ & $\mathbf{z}$ & $\mathbf{F x}$ & $\mathbf{F y}$ & $\mathbf{F z}$ & 3D Position & 3D Force \\
\hline 0.50 & 3.89 & -0.13 & 0.12 & -32.84 & 2.05 & 0.30 & 3.89 & 32.91 \\
1.00 & 3.58 & -0.17 & 0.14 & -28.70 & 2.06 & -0.63 & 3.58 & 28.78 \\
1.50 & 4.29 & -0.16 & 0.21 & -34.44 & 3.34 & -0.49 & 4.29 & 34.61 \\
2.00 & 4.89 & -0.20 & 0.25 & -39.36 & 4.50 & -0.43 & 4.90 & 39.62 \\
2.50 & 4.98 & -0.11 & 0.23 & -38.44 & 5.37 & -3.32 & 4.99 & 38.95 \\
3.00 & 5.09 & -0.17 & 0.25 & -38.67 & 6.26 & -2.70 & 5.10 & 39.26 \\
3.50 & 5.17 & -0.15 & 0.25 & -38.98 & 6.49 & -3.81 & 5.18 & 39.70 \\
4.00 & 5.77 & -0.17 & 0.25 & -43.97 & 6.77 & -4.23 & 5.78 & 44.69 \\
\hline
\end{tabular}

$\mathrm{x}$ : absolute position of the specimen in the $\mathrm{X}$ - axis (mm); $\mathrm{y}$ : absolute position of the specimen in the $\mathrm{Y}$ - axis (mm); $\mathrm{z}$ : absolute position of the specimen in the Z- axis (mm); Fx: absolute forces on the specimen in the X-axis; Fy: absolute forces on the specimen in the Yaxis; Fz: absolute forces on the specimen in the Z-axis. 
Table C-6. Anterior glide 30 degrees ER extension.

\begin{tabular}{ccccccccc}
\hline Normalized Pitch & $\mathbf{x}$ & $\mathbf{y}$ & $\mathbf{z}$ & $\mathbf{F x}$ & $\mathbf{F y}$ & $\mathbf{F z}$ & 3D Position & 3D Force \\
\hline-0.40 & 4.06 & -0.11 & 0.17 & -32.58 & 2.90 & 0.01 & 4.06 & 32.71 \\
-0.90 & 3.64 & -0.09 & 0.15 & -28.80 & 1.95 & 0.23 & 3.64 & 28.87 \\
-1.40 & 3.20 & -0.12 & 0.12 & -25.36 & 1.42 & 0.14 & 3.21 & 25.40 \\
-1.90 & 2.76 & -0.14 & 0.10 & -22.36 & 0.58 & 0.13 & 2.77 & 22.36 \\
-2.40 & 2.51 & -0.17 & 0.09 & -20.47 & 0.55 & 0.46 & 2.51 & 20.48 \\
-2.90 & 2.33 & -0.17 & 0.08 & -20.01 & -0.11 & 1.10 & 2.34 & 20.04 \\
-3.40 & 2.32 & -0.10 & 0.09 & -20.09 & 0.01 & 0.84 & 2.32 & 20.10 \\
-3.90 & 2.24 & -0.20 & 0.09 & -19.98 & -0.56 & 1.45 & 2.25 & 20.04 \\
-4.40 & 2.16 & -0.07 & 0.09 & -19.99 & -0.30 & 1.19 & 2.16 & 20.03 \\
-4.90 & 2.09 & -0.20 & 0.10 & -19.71 & -1.09 & 2.09 & 2.10 & 19.85 \\
-5.40 & 2.10 & -0.17 & 0.10 & -19.80 & -0.47 & 1.74 & 2.11 & 19.88 \\
-5.90 & 2.00 & -0.16 & 0.11 & -19.59 & -1.03 & 2.11 & 2.01 & 19.73 \\
-6.40 & 1.98 & -0.57 & 0.12 & -19.65 & -1.03 & 2.51 & 2.07 & 19.83 \\
\hline
\end{tabular}

$\mathrm{x}$ : absolute position of the specimen in the X-axis (mm); $\mathrm{y}$ : absolute position of the specimen in the $\mathrm{Y}$ - axis (mm); $\mathrm{z}$ : absolute position of the specimen in the Z- axis (mm); Fx: absolute forces on the specimen in the X-axis; Fy: absolute forces on the specimen in the Yaxis; Fz: absolute forces on the specimen in the Z-axis. 
Table C-7. Posterior glide NR flexion.

\begin{tabular}{ccccccccc}
\hline Normalized Pitch & $\mathbf{x}$ & $\mathbf{y}$ & $\mathbf{z}$ & $\mathbf{F x}$ & $\mathbf{F y}$ & $\mathbf{F z}$ & 3D Position & 3D Force \\
\hline 0.50 & -10.00 & -1.96 & -0.25 & 14.87 & 5.29 & -0.73 & 10.19 & 15.80 \\
1.00 & -9.99 & -1.99 & 0.00 & 16.20 & 5.86 & 1.30 & 10.19 & 17.28 \\
1.50 & -10.00 & -2.01 & 0.00 & 16.06 & 6.17 & -0.18 & 10.20 & 17.20 \\
2.00 & -10.00 & -2.00 & 0.00 & 16.41 & 6.73 & 1.11 & 10.20 & 17.78 \\
2.50 & -10.00 & -2.03 & 0.00 & 16.42 & 6.65 & -0.28 & 10.20 & 17.72 \\
3.00 & -10.01 & -2.08 & 0.00 & 17.04 & 7.22 & 0.73 & 10.22 & 18.52 \\
3.50 & -10.00 & -2.09 & 0.00 & 17.21 & 7.00 & -0.64 & 10.22 & 18.59 \\
4.00 & -10.01 & -2.08 & 0.00 & 17.89 & 7.52 & 0.47 & 10.22 & 19.42 \\
4.50 & -10.01 & -2.13 & 0.00 & 18.31 & 7.65 & -1.44 & 10.23 & 19.90 \\
5.00 & -10.00 & -2.11 & 0.00 & 19.24 & 7.78 & -0.15 & 10.22 & 20.75 \\
5.50 & -9.94 & -2.17 & 0.00 & 19.63 & 7.65 & -1.39 & 10.17 & 21.12 \\
6.00 & -9.73 & -2.13 & 0.24 & 18.86 & 7.70 & 1.69 & 9.97 & 20.44 \\
6.50 & -9.64 & -2.13 & 0.25 & 18.98 & 7.39 & 0.63 & 9.87 & 20.38 \\
7.00 & -9.51 & -2.07 & 0.25 & 19.06 & 7.48 & 0.10 & 9.74 & 20.47 \\
7.50 & -9.38 & -2.06 & 0.25 & 19.24 & 7.39 & -0.10 & 9.61 & 20.61 \\
8.00 & -9.26 & -2.04 & 0.25 & 19.25 & 7.00 & -0.02 & 9.49 & 20.48 \\
8.50 & -8.94 & -2.00 & 0.25 & 19.05 & 7.39 & 1.18 & 9.17 & 20.47 \\
9.00 & -8.81 & -2.03 & 0.25 & 19.26 & 7.26 & 0.80 & 9.04 & 20.60 \\
9.50 & -8.66 & -1.96 & 0.25 & 19.22 & 7.17 & 0.66 & 8.88 & 20.52 \\
10.00 & -8.52 & -2.19 & 0.25 & 19.08 & 6.52 & 1.55 & 8.80 & 20.22 \\
\hline
\end{tabular}

$\mathrm{x}$ : absolute position of the specimen in the X-axis (mm); $\mathrm{y}$ : absolute position of the specimen in the $\mathrm{Y}$ - axis (mm); $\mathrm{z}$ : absolute position of the specimen in the Z- axis (mm); Fx: absolute forces on the specimen in the X-axis; Fy: absolute forces on the specimen in the Yaxis; Fz: absolute forces on the specimen in the Z-axis. 
Table C-8. Posterior glide NR extension.

\begin{tabular}{ccccccccc}
\hline Normalized Pitch & $\mathbf{x}$ & $\mathbf{y}$ & $\mathbf{z}$ & $\mathbf{F x}$ & $\mathbf{F y}$ & $\mathbf{F z}$ & 3D Position & 3D Force \\
\hline-0.40 & -10.00 & -2.10 & 0.00 & 14.77 & 8.05 & -1.50 & 10.22 & 16.89 \\
-0.90 & -10.00 & -2.23 & 0.00 & 15.18 & 8.18 & -2.60 & 10.25 & 17.44 \\
-1.40 & -10.00 & -2.31 & 0.00 & 15.73 & 9.23 & -4.41 & 10.26 & 18.76 \\
-1.90 & -9.99 & -2.34 & 0.25 & 14.24 & 9.14 & -3.32 & 10.26 & 17.24 \\
-2.40 & -9.99 & -2.40 & 0.25 & 14.33 & 8.92 & -3.75 & 10.27 & 17.29 \\
-2.90 & -9.99 & -2.40 & 0.25 & 14.35 & 8.70 & -3.75 & 10.27 & 17.19 \\
-3.40 & -9.98 & -2.41 & 0.25 & 14.37 & 8.48 & -3.75 & 10.27 & 17.10 \\
-3.90 & -9.98 & -2.45 & 0.25 & 13.97 & 7.39 & -3.41 & 10.27 & 16.17 \\
-4.40 & -9.98 & -2.45 & 0.25 & 14.15 & 7.78 & -3.63 & 10.28 & 16.55 \\
-4.90 & -9.98 & -2.46 & 0.25 & 12.83 & 7.04 & -6.02 & 10.28 & 15.82 \\
-5.40 & -9.97 & -2.44 & 0.25 & 13.88 & 7.74 & -3.73 & 10.27 & 16.32 \\
-5.90 & -9.97 & -2.46 & 0.25 & 13.93 & 7.57 & -3.07 & 10.27 & 16.14 \\
-6.40 & -9.97 & -2.44 & 0.25 & 13.39 & 7.52 & -4.23 & 10.26 & 15.93 \\
-6.90 & -9.97 & -2.54 & 0.25 & 13.58 & 7.00 & -3.14 & 10.29 & 15.60 \\
-7.40 & -9.96 & -2.48 & 0.25 & 13.14 & 7.39 & -4.40 & 10.27 & 15.71 \\
-7.90 & -9.96 & -2.48 & 0.25 & 12.92 & 7.22 & -4.57 & 10.27 & 15.49 \\
-8.40 & -9.96 & -2.50 & 0.25 & 13.22 & 6.87 & -3.59 & 10.27 & 15.32 \\
-8.90 & -9.96 & -2.48 & 0.25 & 12.84 & 7.08 & -4.80 & 10.26 & 15.43 \\
-9.40 & -9.95 & -2.44 & 0.25 & 12.59 & 7.13 & -5.32 & 10.25 & 15.42 \\
-9.90 & -9.95 & -2.57 & 0.25 & 13.24 & 6.78 & -4.00 & 10.28 & 15.40 \\
\hline
\end{tabular}

$\mathrm{x}$ : absolute position of the specimen in the X-axis (mm); $\mathrm{y}$ : absolute position of the specimen in the $\mathrm{Y}$ - axis (mm); $\mathrm{z}$ : absolute position of the specimen in the Z- axis (mm); Fx: absolute forces on the specimen in the X-axis; Fy: absolute forces on the specimen in the Yaxis; Fz: absolute forces on the specimen in the Z-axis. 
Table C-9. Posterior glide 30 degrees IR flexion.

\begin{tabular}{ccccccccc}
\hline Normalized Pitch & $\mathbf{x}$ & $\mathbf{y}$ & $\mathbf{z}$ & $\mathbf{F x}$ & $\mathbf{F y}$ & $\mathbf{F z}$ & 3D Position & 3D Force \\
\hline 0.50 & -5.36 & -0.84 & 0.00 & 23.10 & 5.20 & 0.34 & 5.43 & 23.67 \\
1.00 & -5.26 & -0.82 & -0.24 & 22.85 & 4.20 & -1.65 & 5.33 & 23.29 \\
1.50 & -5.21 & -0.82 & -0.25 & 23.18 & 4.47 & -1.88 & 5.28 & 23.69 \\
2.00 & -5.10 & -0.76 & -0.23 & 23.15 & 4.27 & -0.59 & 5.17 & 23.55 \\
2.50 & -5.03 & -0.79 & -0.25 & 23.10 & 3.99 & -1.70 & 5.10 & 23.50 \\
3.00 & -4.87 & -0.75 & -0.22 & 23.36 & 4.36 & -0.09 & 4.93 & 23.76 \\
3.50 & -4.80 & -0.73 & -0.25 & 23.02 & 3.80 & -1.44 & 4.86 & 23.38 \\
4.00 & -4.61 & -0.76 & -0.22 & 23.17 & 4.27 & 0.09 & 4.67 & 23.56 \\
4.50 & -4.49 & -0.73 & -0.23 & 22.98 & 3.78 & -1.00 & 4.56 & 23.31 \\
5.00 & -4.34 & -0.74 & -0.17 & 22.89 & 3.96 & 0.87 & 4.41 & 23.25 \\
5.50 & -4.18 & -0.73 & -0.20 & 22.80 & 3.91 & 0.09 & 4.24 & 23.13 \\
6.00 & -3.99 & -0.65 & 0.00 & 22.97 & 4.49 & 3.39 & 4.04 & 23.64 \\
6.50 & -3.86 & -0.66 & 0.00 & 22.89 & 4.36 & 2.83 & 3.92 & 23.48 \\
7.00 & -3.62 & -0.58 & 0.00 & 22.58 & 4.11 & 3.83 & 3.67 & 23.27 \\
7.50 & -3.52 & -0.56 & 0.00 & 22.67 & 4.05 & 3.17 & 3.56 & 23.25 \\
8.00 & -3.39 & -0.58 & 0.00 & 22.54 & 3.87 & 2.64 & 3.44 & 23.02 \\
8.50 & -3.22 & -0.55 & 0.00 & 22.64 & 3.83 & 2.50 & 3.27 & 23.09 \\
9.00 & -3.10 & -0.57 & 0.00 & 22.50 & 3.82 & 2.42 & 3.16 & 22.95 \\
9.50 & -2.95 & -0.49 & 0.00 & 22.56 & 3.70 & 2.32 & 2.99 & 22.98 \\
10.00 & -2.80 & -1.45 & 0.00 & 22.59 & 3.45 & 1.68 & 3.16 & 22.91 \\
\hline
\end{tabular}

$\mathrm{x}$ : absolute position of the specimen in the X-axis (mm); $\mathrm{y}$ : absolute position of the specimen in the $\mathrm{Y}$ - axis (mm); $\mathrm{z}$ : absolute position of the specimen in the Z- axis (mm); Fx: absolute forces on the specimen in the X-axis; Fy: absolute forces on the specimen in the Yaxis; Fz: absolute forces on the specimen in the Z-axis. 
Table C-10. Posterior glide 30 degrees IR extension.

\begin{tabular}{ccccccccc}
\hline Normalized Pitch & $\mathbf{x}$ & $\mathbf{y}$ & $\mathbf{z}$ & $\mathbf{F x}$ & $\mathbf{F y}$ & $\mathbf{F z}$ & 3D Position & 3D Force \\
\hline-0.40 & -5.26 & -0.82 & 0.00 & 22.96 & 5.58 & -0.25 & 5.32 & 23.63 \\
-0.90 & -4.96 & -0.89 & 0.00 & 23.35 & 5.55 & -0.28 & 5.04 & 24.00 \\
-1.40 & -4.73 & -0.83 & 0.00 & 23.47 & 5.56 & -1.97 & 4.80 & 24.20 \\
-1.90 & -4.50 & -0.89 & 0.00 & 23.43 & 5.54 & -3.93 & 4.59 & 24.40 \\
-2.40 & -4.21 & -0.84 & 0.20 & 21.93 & 5.61 & -2.76 & 4.30 & 22.80 \\
-2.90 & -4.00 & -0.83 & 0.25 & 21.32 & 5.47 & -3.88 & 4.09 & 22.35 \\
-3.40 & -3.73 & -0.74 & 0.25 & 20.82 & 4.95 & -5.01 & 3.81 & 21.98 \\
-3.90 & -3.41 & -0.74 & 0.25 & 20.61 & 4.93 & -5.35 & 3.49 & 21.86 \\
-4.40 & -3.19 & -1.57 & 0.25 & 20.05 & 4.20 & -6.02 & 3.56 & 21.35 \\
\hline
\end{tabular}

$\mathrm{x}$ : absolute position of the specimen in the $\mathrm{X}$ - axis $(\mathrm{mm})$; $\mathrm{y}$ : absolute position of the specimen in the $\mathrm{Y}$ - axis (mm); $\mathrm{z}$ : absolute position of the specimen in the Z- axis (mm); Fx: absolute forces on the specimen in the X-axis; Fy: absolute forces on the specimen in the Yaxis; Fz: absolute forces on the specimen in the Z-axis. 
Table C-11. Posterior glide 30 degrees ER flexion.

\begin{tabular}{ccccccccc}
\hline Normalized Pitch & $\mathbf{x}$ & $\mathbf{y}$ & $\mathbf{z}$ & $\mathbf{F x}$ & $\mathbf{F y}$ & $\mathbf{F z}$ & 3D Position & 3D Force \\
\hline 0.50 & -2.43 & -0.18 & 0.00 & 18.54 & 2.01 & -1.79 & 2.44 & 18.74 \\
1.00 & -2.32 & -0.23 & 0.09 & 18.61 & 2.21 & 0.73 & 2.34 & 18.75 \\
1.50 & -2.30 & -0.23 & 0.07 & 18.58 & 2.49 & -0.77 & 2.32 & 18.77 \\
2.00 & -2.34 & -0.23 & 0.11 & 18.38 & 2.30 & 1.48 & 2.35 & 18.58 \\
2.50 & -2.22 & -0.29 & 0.08 & 18.62 & 2.70 & -0.31 & 2.24 & 18.81 \\
3.00 & -2.34 & -0.27 & 0.14 & 18.22 & 2.14 & 2.11 & 2.36 & 18.47 \\
3.50 & -2.26 & -0.32 & 0.12 & 18.59 & 2.49 & 0.14 & 2.29 & 18.75 \\
4.00 & -2.33 & -0.51 & 0.18 & 18.44 & 2.00 & 2.84 & 2.40 & 18.76 \\
\hline
\end{tabular}

$\mathrm{x}$ : absolute position of the specimen in the $\mathrm{X}$ - axis (mm); $\mathrm{y}$ : absolute position of the specimen in the $\mathrm{Y}$ - axis (mm); $\mathrm{z}$ : absolute position of the specimen in the Z- axis (mm); Fx: absolute forces on the specimen in the X-axis; Fy: absolute forces on the specimen in the Yaxis; Fz: absolute forces on the specimen in the Z-axis. 
Table C-12. Posterior glide 30 degrees ER extension.

\begin{tabular}{ccccccccc}
\hline Normalized Pitch & $\mathbf{x}$ & $\mathbf{y}$ & $\mathbf{z}$ & $\mathbf{F x}$ & $\mathbf{F y}$ & $\mathbf{F z}$ & 3D Position & 3D Force \\
\hline-0.40 & -2.23 & -0.22 & 0.00 & 18.52 & 2.72 & -1.00 & 2.24 & 18.75 \\
-0.90 & -2.30 & -0.18 & 0.00 & 18.44 & 2.48 & -1.43 & 2.31 & 18.66 \\
-1.40 & -2.18 & -0.19 & 0.00 & 18.62 & 3.14 & -1.07 & 2.18 & 18.91 \\
-1.90 & -2.27 & -0.17 & 0.00 & 18.60 & 2.41 & -0.36 & 2.28 & 18.76 \\
-2.40 & -2.33 & -0.17 & 0.00 & 19.93 & 3.12 & 0.04 & 2.34 & 20.17 \\
-2.90 & -2.25 & -0.21 & 0.00 & 18.77 & 2.48 & 0.49 & 2.26 & 18.94 \\
-3.40 & -2.28 & -0.20 & -0.06 & 18.35 & 2.46 & -1.79 & 2.29 & 18.60 \\
-3.90 & -2.67 & -0.20 & -0.09 & 21.60 & 2.60 & -2.66 & 2.68 & 21.91 \\
-4.40 & -2.66 & -0.32 & -0.10 & 21.23 & 2.54 & -2.47 & 2.68 & 21.52 \\
-4.90 & -3.20 & -0.33 & -0.16 & 24.29 & 2.60 & -2.92 & 3.22 & 24.60 \\
-5.40 & -3.35 & -0.34 & -0.20 & 25.18 & 2.75 & -2.69 & 3.37 & 25.47 \\
-5.90 & -3.41 & -0.49 & -0.24 & 25.19 & 2.62 & -2.87 & 3.45 & 25.49 \\
-6.40 & -3.78 & -0.57 & -0.25 & 27.31 & 2.66 & -1.88 & 3.83 & 27.50 \\
\hline
\end{tabular}

$\mathrm{x}$ : absolute position of the specimen in the X-axis (mm); $\mathrm{y}$ : absolute position of the specimen in the $\mathrm{Y}$ - axis (mm); $\mathrm{z}$ : absolute position of the specimen in the Z- axis (mm); Fx: absolute forces on the specimen in the X-axis; Fy: absolute forces on the specimen in the Yaxis; Fz: absolute forces on the specimen in the Z-axis. 
Table C-13. Inferior glide NR flexion.

\begin{tabular}{ccccccccc}
\hline Normalized Pitch & $\mathbf{x}$ & $\mathbf{y}$ & $\mathbf{z}$ & $\mathbf{F x}$ & $\mathbf{F y}$ & $\mathbf{F z}$ & 3D Position & 3D Force \\
\hline 0.50 & -0.25 & -1.68 & 3.33 & 0.78 & 4.50 & 13.04 & 3.73 & 13.82 \\
1.00 & -0.14 & -1.81 & 4.60 & -0.29 & 8.09 & 12.77 & 4.95 & 15.12 \\
1.50 & -0.35 & -1.89 & 5.16 & 0.27 & 8.35 & 12.43 & 5.50 & 14.98 \\
2.00 & -0.03 & -1.92 & 5.46 & -0.45 & 8.57 & 13.64 & 5.79 & 16.11 \\
2.50 & -0.30 & -1.99 & 5.59 & 0.15 & 8.09 & 13.05 & 5.94 & 15.35 \\
3.00 & -0.54 & -2.00 & 5.82 & 0.73 & 8.00 & 12.43 & 6.17 & 14.80 \\
3.50 & -0.25 & -2.03 & 5.76 & 0.17 & 8.22 & 13.33 & 6.11 & 15.66 \\
4.00 & -0.25 & -2.05 & 5.99 & 0.25 & 7.87 & 13.12 & 6.33 & 15.30 \\
4.50 & -0.25 & -2.03 & 6.10 & 0.18 & 8.05 & 13.04 & 6.43 & 15.32 \\
5.00 & -0.25 & -2.14 & 6.01 & 0.41 & 8.18 & 13.12 & 6.38 & 15.46 \\
5.50 & -0.25 & -2.26 & 6.30 & 0.37 & 8.09 & 13.26 & 6.70 & 15.53 \\
6.00 & -0.25 & -2.25 & 6.94 & 0.51 & 8.48 & 12.89 & 7.30 & 15.44 \\
6.50 & -0.25 & -2.29 & 7.15 & 0.54 & 8.57 & 13.26 & 7.52 & 15.80 \\
7.00 & -0.25 & -2.26 & 7.37 & 0.66 & 8.61 & 13.12 & 7.71 & 15.71 \\
7.50 & -0.49 & -2.27 & 7.27 & 1.35 & 8.31 & 12.70 & 7.63 & 15.23 \\
8.00 & -0.50 & -2.30 & 7.00 & 1.26 & 8.09 & 12.85 & 7.38 & 15.23 \\
8.50 & -0.25 & -2.31 & 7.28 & 1.07 & 8.48 & 13.68 & 7.64 & 16.14 \\
9.00 & -0.25 & -2.30 & 7.48 & 1.10 & 8.48 & 13.63 & 7.83 & 16.09 \\
9.50 & -0.25 & -2.35 & 7.56 & 1.22 & 8.70 & 13.77 & 7.92 & 16.33 \\
10.00 & -0.25 & -2.47 & 7.72 & 1.43 & 8.88 & 13.92 & 8.11 & 16.57 \\
\hline
\end{tabular}

$\mathrm{x}$ : absolute position of the specimen in the $\mathrm{X}$ - axis $(\mathrm{mm})$; $\mathrm{y}$ : absolute position of the specimen in the $\mathrm{Y}$ - axis (mm); $\mathrm{z}$ : absolute position of the specimen in the Z- axis (mm); Fx: absolute forces on the specimen in the X-axis; Fy: absolute forces on the specimen in the Yaxis; Fz: absolute forces on the specimen in the Z-axis. 
Table C-14. Inferior glide NR extension.

\begin{tabular}{ccccccccc}
\hline Normalized Pitch & $\mathbf{x}$ & $\mathbf{y}$ & $\mathbf{z}$ & $\mathbf{F x}$ & $\mathbf{F y}$ & $\mathbf{F z}$ & 3D Position & 3D Force \\
\hline-0.40 & -0.50 & -2.08 & 5.95 & 0.44 & 7.96 & 12.42 & 6.33 & 14.75 \\
-0.90 & -0.75 & -2.27 & 6.29 & 1.12 & 8.00 & 12.58 & 6.73 & 14.95 \\
-1.40 & -0.91 & -2.26 & 7.27 & 0.86 & 8.26 & 11.96 & 7.67 & 14.57 \\
-1.90 & -0.25 & -2.31 & 7.36 & -0.34 & 8.62 & 13.66 & 7.71 & 16.15 \\
-2.40 & -0.25 & -2.27 & 7.47 & -0.35 & 8.57 & 13.54 & 7.81 & 16.03 \\
-2.90 & -0.25 & -2.42 & 7.19 & -0.14 & 8.44 & 13.58 & 7.59 & 15.99 \\
-3.40 & -0.68 & -2.44 & 7.86 & -0.26 & 7.92 & 12.63 & 8.26 & 14.91 \\
-3.90 & -0.15 & -2.30 & 7.63 & -0.27 & 7.30 & 13.34 & 7.97 & 15.21 \\
-4.40 & -0.20 & -2.32 & 7.78 & -0.47 & 7.52 & 13.28 & 8.12 & 15.27 \\
-4.90 & 0.25 & -1.13 & 3.24 & 2.07 & 4.90 & 13.91 & 3.44 & 14.89 \\
-5.40 & 0.25 & -1.10 & 3.69 & 1.38 & 5.47 & 13.66 & 3.86 & 14.78 \\
-5.90 & 0.18 & -1.19 & 3.66 & 1.50 & 5.77 & 13.69 & 3.85 & 14.94 \\
-6.40 & 0.04 & -1.21 & 3.89 & 1.56 & 6.30 & 13.91 & 4.07 & 15.34 \\
-6.90 & 0.22 & -1.05 & 3.21 & 1.72 & 5.07 & 13.84 & 3.38 & 14.84 \\
-7.40 & 0.06 & -1.07 & 3.56 & 1.80 & 5.86 & 13.83 & 3.71 & 15.13 \\
-7.90 & 0.25 & -1.03 & 3.57 & 0.98 & 5.73 & 14.04 & 3.72 & 15.19 \\
-8.40 & 0.14 & -1.04 & 3.10 & 2.06 & 5.20 & 13.97 & 3.27 & 15.05 \\
-8.90 & 0.25 & -1.06 & 3.44 & 1.23 & 5.86 & 14.37 & 3.61 & 15.57 \\
-9.40 & 0.24 & -1.03 & 3.54 & 0.88 & 5.82 & 14.13 & 3.69 & 15.30 \\
-9.90 & 0.25 & -2.51 & 3.18 & 1.47 & 4.94 & 14.26 & 4.06 & 15.16 \\
\hline
\end{tabular}

$\mathrm{x}$ : absolute position of the specimen in the $\mathrm{X}$ - axis $(\mathrm{mm})$; $\mathrm{y}$ : absolute position of the specimen in the $\mathrm{Y}$ - axis (mm); $\mathrm{z}$ : absolute position of the specimen in the Z- axis (mm); Fx: absolute forces on the specimen in the X-axis; Fy: absolute forces on the specimen in the Yaxis; Fz: absolute forces on the specimen in the Z-axis. 
Table C-15. Inferior glide 30 degrees IR flexion.

\begin{tabular}{ccccccccc}
\hline Normalized Pitch & $\mathbf{x}$ & $\mathbf{y}$ & $\mathbf{z}$ & $\mathbf{F x}$ & $\mathbf{F y}$ & $\mathbf{F z}$ & 3D Position & 3D Force \\
\hline 0.50 & -0.25 & -0.72 & 2.59 & 3.06 & 6.41 & 12.27 & 2.70 & 14.18 \\
1.00 & -0.50 & -0.71 & 2.72 & 4.32 & 6.33 & 12.75 & 2.86 & 14.87 \\
1.50 & -0.50 & -0.75 & 2.74 & 4.44 & 6.40 & 13.01 & 2.88 & 15.16 \\
2.00 & -1.00 & -0.74 & 2.85 & 5.91 & 6.40 & 12.53 & 3.10 & 15.26 \\
2.50 & -0.50 & -0.76 & 2.87 & 4.47 & 6.18 & 13.58 & 3.01 & 15.58 \\
3.00 & -0.75 & -0.75 & 2.89 & 5.31 & 6.21 & 12.88 & 3.07 & 15.25 \\
3.50 & -0.50 & -0.82 & 2.88 & 4.66 & 6.10 & 13.29 & 3.04 & 15.34 \\
4.00 & -1.00 & -0.80 & 2.93 & 6.77 & 6.24 & 13.13 & 3.19 & 16.03 \\
4.50 & -0.50 & -0.83 & 2.91 & 4.95 & 5.99 & 13.63 & 3.06 & 15.69 \\
5.00 & -0.74 & -0.81 & 2.92 & 6.20 & 5.93 & 13.26 & 3.12 & 15.79 \\
5.50 & -0.50 & -0.67 & 2.89 & 5.50 & 5.69 & 13.74 & 3.00 & 15.86 \\
6.00 & -0.50 & -1.07 & 2.86 & 5.49 & 5.64 & 13.40 & 3.09 & 15.55 \\
\hline
\end{tabular}

$\mathrm{x}$ : absolute position of the specimen in the X-axis (mm); $\mathrm{y}$ : absolute position of the specimen in the $\mathrm{Y}$ - axis (mm); $\mathrm{z}$ : absolute position of the specimen in the Z-axis (mm); Fx: absolute forces on the specimen in the X-axis; Fy: absolute forces on the specimen in the Yaxis; Fz: absolute forces on the specimen in the Z-axis. 
Table C-16. Inferior glide 30 degrees IR extension.

\begin{tabular}{ccccccccc}
\hline Normalized Pitch & $\mathbf{x}$ & $\mathbf{y}$ & $\mathbf{z}$ & $\mathbf{F x}$ & $\mathbf{F y}$ & $\mathbf{F z}$ & 3D Position & 3D Force \\
\hline-0.40 & -0.25 & -0.57 & 2.72 & 1.98 & 5.98 & 13.02 & 2.79 & 15.44 \\
-0.90 & -0.25 & -0.64 & 2.82 & 2.45 & 6.23 & 13.78 & 2.90 & 16.62 \\
-1.40 & -0.25 & -0.75 & 2.99 & 2.10 & 6.36 & 14.08 & 3.09 & 17.93 \\
-1.90 & -0.50 & -0.78 & 3.13 & 2.62 & 6.31 & 13.08 & 3.26 & 18.82 \\
-2.40 & -0.74 & -0.80 & 3.29 & 3.48 & 6.04 & 13.07 & 3.47 & 20.53 \\
-2.90 & -0.75 & -0.86 & 3.39 & 3.69 & 5.99 & 13.43 & 3.58 & 22.39 \\
-3.40 & -0.75 & -1.53 & 3.51 & 3.87 & 5.98 & 11.25 & 3.90 & 22.00 \\
\hline
\end{tabular}

$\mathrm{x}$ : absolute position of the specimen in the $\mathrm{X}$ - axis $(\mathrm{mm})$; $\mathrm{y}$ : absolute position of the specimen in the $\mathrm{Y}$ - axis (mm); $\mathrm{z}$ : absolute position of the specimen in the Z- axis (mm); Fx: absolute forces on the specimen in the X-axis; Fy: absolute forces on the specimen in the Yaxis; Fz: absolute forces on the specimen in the Z-axis. 
Table C-17. Inferior glide 30 degrees ER flexion.

\begin{tabular}{ccccccccc}
\hline Normalized Pitch & $\mathbf{x}$ & $\mathbf{y}$ & $\mathbf{z}$ & $\mathbf{F x}$ & $\mathbf{F y}$ & $\mathbf{F z}$ & 3D Position & 3D Force \\
\hline 0.50 & 0.49 & -0.36 & 1.18 & -1.42 & 3.27 & 12.41 & 1.33 & 12.91 \\
1.00 & 0.50 & -0.33 & 1.29 & -1.11 & 3.86 & 12.53 & 1.42 & 13.16 \\
1.50 & 0.74 & -0.41 & 1.35 & -2.25 & 4.04 & 12.98 & 1.59 & 13.78 \\
2.00 & 0.75 & -0.39 & 1.41 & -1.07 & 4.72 & 12.39 & 1.64 & 13.30 \\
2.50 & 0.25 & -0.39 & 1.37 & -0.57 & 4.37 & 14.81 & 1.44 & 15.46 \\
3.00 & 0.50 & -0.55 & 1.42 & 0.02 & 4.96 & 13.84 & 1.61 & 14.70 \\
3.50 & 0.74 & -0.39 & 1.46 & -1.94 & 4.75 & 13.64 & 1.68 & 14.57 \\
4.00 & 0.75 & -0.39 & 1.50 & -0.74 & 5.34 & 13.10 & 1.72 & 14.16 \\
\hline
\end{tabular}

$\mathrm{x}$ : absolute position of the specimen in the $\mathrm{X}$ - axis (mm); $\mathrm{y}$ : absolute position of the specimen in the $\mathrm{Y}$ - axis (mm); $\mathrm{z}$ : absolute position of the specimen in the Z- axis (mm); Fx: absolute forces on the specimen in the X-axis; Fy: absolute forces on the specimen in the Yaxis; Fz: absolute forces on the specimen in the Z-axis. 
Table C-18. Inferior glide 30 degrees ER extension.

\begin{tabular}{ccccccccc}
\hline Normalized Pitch & $\mathbf{x}$ & $\mathbf{y}$ & $\mathbf{z}$ & $\mathbf{F x}$ & $\mathbf{F y}$ & $\mathbf{F z}$ & 3D Position & 3D Force \\
\hline-0.40 & 0.25 & -0.17 & 0.63 & -1.89 & 2.70 & 10.51 & 0.70 & 11.01 \\
-0.90 & 0.25 & -0.20 & 1.00 & -0.39 & 4.14 & 14.53 & 1.05 & 15.11 \\
-1.40 & 0.25 & -0.15 & 0.97 & -0.22 & 4.32 & 14.48 & 1.02 & 15.11 \\
-1.90 & 0.49 & -0.17 & 0.97 & -1.86 & 4.31 & 14.28 & 1.10 & 15.03 \\
-2.40 & 0.25 & -0.17 & 0.91 & -1.20 & 3.85 & 14.84 & 0.96 & 15.38 \\
-2.90 & 0.25 & -0.11 & 0.59 & -1.75 & 2.98 & 10.51 & 0.65 & 11.06 \\
-3.40 & 0.25 & -0.10 & 0.60 & -1.74 & 2.93 & 12.02 & 0.66 & 12.49 \\
-3.90 & 0.25 & -0.16 & 0.60 & -1.02 & 3.06 & 12.52 & 0.67 & 12.93 \\
-4.40 & 0.25 & -0.54 & 0.57 & -1.66 & 2.86 & 13.11 & 0.83 & 13.52 \\
\hline
\end{tabular}

$\mathrm{x}$ : absolute position of the specimen in the $\mathrm{X}$ - axis $(\mathrm{mm})$; $\mathrm{y}$ : absolute position of the specimen in the $\mathrm{Y}$ - axis (mm); $\mathrm{z}$ : absolute position of the specimen in the Z- axis (mm); Fx: absolute forces on the specimen in the X-axis; Fy: absolute forces on the specimen in the Yaxis; Fz: absolute forces on the specimen in the Z-axis. 


\section{VITA}

Hunter Johnson Smith was born in Clarksville, Tennessee in 1988. He was raised in Newbern, Tennessee where he attended Dyer County High School. Upon graduation in 2006, he enrolled into the University of Tennessee at Martin where he graduated with a Bachelor of Science in Mechanical Engineering. After graduation in May 2010, he was accepted into the University of Tennessee Health Science Center and University of Memphis Joint Program in Biomedical Engineering. Hunter entered the program as a student of Dr. Denis DiAngelo to study joint biomechanics. In December 2013, Hunter earned a Master of Science in Biomedical Engineering focusing on the biomechanis of the glenohumeral joint. 